\title{
Exact asymptotic formulae of the stationary distribution of a discrete-time two-dimensional QBD process
}

\author{
Toshihisa Ozawa ${ }^{\dagger}$ and Masahiro Kobayashi ${ }^{\dagger \dagger}$ \\ ${ }^{\dagger}$ Faculty of Business Administration, Komazawa University \\ ${ }^{\dagger \dagger}$ Department of Mathematical Science, Tokai University \\ ${ }^{\dagger}$ 1-23-1 Komazawa, Setagaya-ku, Tokyo 154-8525, Japan \\ E-mail: †toshi@komazawa-u.ac.jp, ${ }^{\dagger} \mathrm{m}$ _kobayashi@tsc.u-tokai.a.c.jp
}

September 12, 2018

\begin{abstract}
We consider a discrete-time two-dimensional process $\left\{\left(L_{1, n}, L_{2, n}\right)\right\}$ on $\mathbb{Z}_{+}^{2}$ with a supplemental process $\left\{J_{n}\right\}$ on a finite set, where individual processes $\left\{L_{1, n}\right\}$ and $\left\{L_{2, n}\right\}$ are both skip free. We assume that the joint process $\left\{Y_{n}\right\}=\left\{\left(L_{1, n}, L_{2, n}, J_{n}\right)\right\}$ is Markovian and that the transition probabilities of the two-dimensional process $\left\{\left(L_{1, n}, L_{2, n}\right)\right\}$ are modulated depending on the state of the background process $\left\{J_{n}\right\}$. This modulation is space homogeneous except for the boundaries of $\mathbb{Z}_{+}^{2}$. We call this process a discrete-time two-dimensional quasi-birth-and-death (2D-QBD) process and obtain the exact asymptotic formulae of the stationary distribution in the coordinate directions.
\end{abstract}

Key wards: quasi-birth-and-death process, stationary distribution, asymptotic property, matrix analytic method, two-dimensional reflecting random walk

Mathematical Subject Classification: 60J10, 60K25

\section{Introduction}

We deal with a discrete-time two-dimensional process $\left\{\left(L_{1, n}, L_{2, n}\right)\right\}$ on $\mathbb{Z}_{+}^{2}$ with a supplemental process $\left\{J_{n}\right\}$ on a finite set $S_{0}$. We call the supplemental process a phase process. Individual processes $\left\{L_{1, n}\right\}$ and $\left\{L_{2, n}\right\}$ are skip free, which means that their increments take values in $\{-1,0,1\}$. We assume that the joint process $\left\{\boldsymbol{Y}_{n}\right\}=\left\{\left(L_{1, n}, L_{2, n}, J_{n}\right)\right\}$ is Markovian and that the transition probabilities of the two-dimensional process $\left\{\left(L_{1, n}, L_{2, n}\right)\right\}$ are modulated depending on the state of the phase process $\left\{J_{n}\right\}$. This modulation is space homogeneous except for the boundaries of $\mathbb{Z}_{+}^{2}$. Since a one-dimensional version of this model is called a discrete-time quasi-birth-and-death (QBD) process (see, for example, Latouche and Ramaswami [10]), we call it a discrete-time twodimensional quasi-birth-and-death (2D-QBD) process [18]. Note that a 2D-QBD process is also a two-dimensional skip-free Markov modulated reflecting random walk (MMRRW) [19] and stochastic models arising from various Markovian queueing networks with two nodes such as generalized two-node Jakson networks with Markovian arrival processes and phase-type service processes can be represented as 2D-QBD processes (see, for example, Ozawa [18] and [19]).

Assume that the 2D-QBD process $\left\{\boldsymbol{Y}_{n}\right\}$ is irreducible, aperiodic and positive recurrent, and denote by $\boldsymbol{\nu}=\left(\boldsymbol{\nu}_{k, l},(k, l) \in \mathbb{Z}^{2}\right)$ its stationary distribution, where $\boldsymbol{\nu}_{k, l}=\left(\nu_{k, l, j}, j \in S_{0}\right)$ and 
$\nu_{k, l, j}=\lim _{n \rightarrow \infty} \mathbb{P}\left(\boldsymbol{Y}_{n}=(k, l, j)\right)$. Our aim is to reveal asymptotic properties of the stationary distribution $\boldsymbol{\nu}$, especially, to obtain the directional exact asymptotic formulae $h_{1}(k)$ and $h_{2}(k)$ that satisfy, for some nonzero vector $\boldsymbol{c}_{1}$ and $\boldsymbol{c}_{2}$,

$$
\lim _{k \rightarrow \infty} \frac{\boldsymbol{\nu}_{k, 0}}{h_{1}(k)}=\boldsymbol{c}_{1}, \quad \lim _{k \rightarrow \infty} \frac{\boldsymbol{\nu}_{0, k}}{h_{2}(k)}=\boldsymbol{c}_{2} .
$$

A 2D-QBD process without a phase process is a two-dimensional skip-free reflecting random walk (RRW), which is called a double QBD process in Miyazawa [14, and the directional exact asymptotic formulae of the stationary distribution of the double QBD process, denoted by $h_{1}^{\prime}(k)$ and $h_{2}^{\prime}(k)$, are obtained in Kobayashi and Miyazawa [8]. For $i \in\{1,2\}, h_{i}^{\prime}(k)$ is given in a form

$$
h_{i}^{\prime}(k)=k^{\alpha_{i}^{\prime}-1}\left(r_{i}^{\prime}\right)^{-k},
$$

where $\alpha_{i}^{\prime} \in\left\{-\frac{1}{2}, \frac{1}{2}, 1,2\right\}$ and $r_{i}^{\prime}$ is the geometric decay rate satisfying $r_{i}^{\prime}>1$. We will demonstrate that, under certain conditions, the same results also hold for the 2D-QBD process, i.e., the directional exact asymptotic formulae $h_{i}(k), i=1,2$, are given in a form

$$
h_{i}(k)=k^{\alpha_{i}-1} r_{i}^{-k}
$$

where $\alpha_{i} \in\left\{-\frac{1}{2}, \frac{1}{2}, 1,2\right\}$ and $r_{i}>1$.

While there are several possible approaches for getting asymptotic properties of the stationary distribution (see Miyazawa [15]), we shall adopt an analytic function approach using the convergence domain, which has been used in Kobayashi and Miyazawa 8]. Let $\varphi_{1}(z)$ be the generating function defined as $\boldsymbol{\varphi}_{1}(z)=\sum_{k=0}^{\infty} \boldsymbol{\nu}_{k, 0} z^{k}$, where $z$ is a complex variable, and let $\boldsymbol{\varphi}_{2}(z)$ be defined analogously. By Cauchy's criterion, we see that the radius of convergence of $\varphi_{1}(z)$ and that of $\varphi_{2}(z)$ are respectively given by the directional geometric decay rates $r_{1}$ and $r_{2}$ of the stationary distribution $\boldsymbol{\nu}$, which have already been obtained in Ozawa [18]. Therefore, in order to obtain the exact asymptotic formula $h_{1}(k)$ (resp. $\left.h_{2}(k)\right)$, it suffices to analytically extend $\boldsymbol{\varphi}_{1}(z)$ (resp. $\left.\boldsymbol{\varphi}_{2}(z)\right)$ beyond its convergence domain and clarify the singularities of $\varphi_{1}(z)$ (resp. $\left.\varphi_{2}(z)\right)$ on the circle $|z|=r_{1}$ (resp. $|z|=r_{2}$ ). To this end, we use the following key expression (see Lemma 3.3):

$$
\begin{gathered}
\boldsymbol{\varphi}_{1}(z)=\left\{\sum_{j=1}^{\infty} \boldsymbol{\nu}_{0, j}\left(A_{*,-1}^{(2)}(z)+A_{*, 0}^{(2)}(z) G_{1}(z)+A_{*, 1}^{(2)}(z) G_{1}(z)^{2}\right) G_{1}(z)^{j-1}-\sum_{j=1}^{\infty} \boldsymbol{\nu}_{0, j} G_{1}(z)^{j}\right. \\
\left.\left.+\boldsymbol{\nu}_{0,0}\left(A_{*, 0}^{(0)}(z)+A_{*, 1}^{(0)}(z) G_{1}(z)-I\right)\right\}\left(I-A_{*, 0}^{(1)}(z)-A_{*, 1}^{(1)}(z) G_{1}(z)\right)\right)^{-1}
\end{gathered}
$$

where each $A_{*, k}^{(l)}(z)$ is a matrix whose entries are Laurent polynomials in $z ; G_{1}(z)$ is a solution to the following matrix quadratic equation of $X$ :

$$
A_{*,-1}(z)+\left(A_{*, 0}(z)-I\right) X+A_{*, 1}(z) X^{2}=O
$$

where each $A_{*, k}(z)$ is also a matrix whose entries are Laurent polynomials in $z$. A similar expression also holds for $\varphi_{2}(z)$. The key expression of $\varphi_{1}(z)$ corresponds to equation (29) in Kobayashi and Miyazawa [8], and $G_{1}(z)$ corresponds to a so-called G-matrix of QBD process (see, for example, Latouche and Ramaswami [10]). We first define this matrix function $G_{1}(z)$ on an annular domain on the complex plane, then analytically extend it beyond the annular domain. Using the key expression above and the extended $G_{1}(z)$, we clarify the singularities of $\varphi_{1}(z)$ on the circle $|z|=r_{1}$. 
The singularities of $\varphi_{2}(z)$ on the circle $|z|=r_{2}$ can also be clarified in the same way. Note that, under the assumptions that we state in Section $2, \varphi_{1}(z)$ (resp. $\varphi_{2}(z)$ ) has just one singularity $z=r_{1}$ (resp. $z=r_{2}$ ) on the circle $|z|=r_{1}$ (resp. $|z|=r_{2}$ ), and the singularity is a pole and/or branch point.

The rest of the paper is organized as follows. In Section 2, the 2D-QBD process we consider is described in detail and our main results are stated. In Section 3, we consider the generating function of the stationary distribution and derive the key expressions mentioned above. In Section 4. we redefine the matrix function $G_{1}(z)$ on an annular domain and analytically extend it. The asymptotic formulae $h_{1}(k)$ and $h_{2}(k)$ are obtained in Section 5 .

\section{Preliminary and main results}

Before describing the model, we present several notations used in the paper. $\mathbb{R}$ is the set of all real numbers and $\mathbb{R}_{+}$that of all nonnegative real numbers. $\mathbb{Z}$ is the set of all integers and $\mathbb{Z}_{+}$that of all nonnegative integers. $\mathbb{N}$ is the set of all positive integers. A set $\mathbb{H}$ is defined as $\mathbb{H}=\{-1,0,1\}$ and $\mathbb{H}_{+}$as $\mathbb{H}_{+}=\{0,1\}$. $\mathbb{C}$ is the set of all complex numbers. For $a, b \in \mathbb{R}_{+}, \mathbb{C}[a, b]$ and $\mathbb{C}[a, b)$ are defined as $\mathbb{C}[a, b]=\{z \in \mathbb{C}: a \leq|z| \leq b\}$ and $\mathbb{C}[a, b)=\{z \in \mathbb{C}: a \leq|z|<b\}$, respectively. $\mathbb{C}(a, b]$ and $\mathbb{C}(a, b)$ are analogously defined. For $z \in \mathbb{C}$ and $r \in \mathbb{R}_{+}, \Delta(z, r)$ is the open disc of center $z$ and radius $r . \bar{\Delta}(z, r)$ and $\partial \Delta(z, r)$ are the closed disc and circle of the same center and radius, respectively. We denote by $\Delta_{r}$ the open disc with center 0 and radius $r$, i.e., $\Delta_{r}=\Delta(0, r)$. For a matrix $A=\left(a_{i j}\right)$, we denote by $[A]_{i, j}$ the $(i, j)$-entry of $A$. The transpose of a matrix $A$ is denoted by $A^{\top}$. We denote by $\operatorname{spr}(A)$ the spectral radius of $A$, which is the maximum modulus of eigenvalue of $A$. We denote by $|A|$ the matrix each of whose entries is the modulus of the corresponding entry of $A$, i.e., $|A|=\left(\left|a_{i j}\right|\right)$. $O$ is a matrix of 0 's, $\mathbf{1}$ is a column vector of 1 's and $\mathbf{0}$ is a column vector of 0 's; their dimensions are determined in context. $I$ is the identity matrix. For a $k \times l$ matrix $A=\left(\begin{array}{llll}\boldsymbol{a}_{1} & \boldsymbol{a}_{2} & \cdots & \boldsymbol{a}_{l}\end{array}\right), \operatorname{vec}(A)$ is a $k l \times 1$ vector defined as

$$
\operatorname{vec}(A)=\left(\begin{array}{c}
\boldsymbol{a}_{1} \\
\boldsymbol{a}_{2} \\
\vdots \\
\boldsymbol{a}_{k}
\end{array}\right) .
$$

For matrices $A, B$ and $C$, the identity $\operatorname{vec}(A B C)=\left(C^{\top} \otimes A\right) \operatorname{vec}(B)$ holds (see, for example, Horn and Johnson [6]).

\subsection{Two-dimensional quasi-birth-and-death process}

Consider a two-dimensional process $\left\{\left(X_{1, n}, X_{2, n}\right)\right\}$ on $\mathbb{Z}_{+}^{2}$ and a background process $\left\{J_{n}\right\}$ on a finite state space $S_{0}$, where we denote by $s_{0}$ the number of elements of $S_{0}$, i.e., $S_{0}=\left\{1,2, \ldots, s_{0}\right\}$. We assume that both $\left\{X_{1, n}\right\}$ and $\left\{X_{2, n}\right\}$ are skip free and that the joint process $\left\{\boldsymbol{Y}_{n}\right\}=\left\{\left(X_{1, n}, X_{2, n}, J_{n}\right)\right\}$ is Markovian. To be precise, $\left\{\boldsymbol{Y}_{n}\right\}$ is a discrete-time Markov chain on the state space $\mathcal{S}=\mathbb{Z}_{+}^{2} \times S_{0}$ and the transition probability matrix

$$
P=\left(p_{\left(x_{1}, x_{2}, j\right),\left(x_{1}^{\prime}, x_{2}^{\prime}, j^{\prime}\right)},\left(x_{1}, x_{2}, j\right),\left(x_{1}^{\prime}, x_{2}^{\prime}, j^{\prime}\right) \in \mathbb{Z}_{+}^{2} \times S_{0}\right),
$$


where $p_{\left(x_{1}, x_{2}, j\right),\left(x_{1}^{\prime}, x_{2}^{\prime}, j^{\prime}\right)}=\mathbb{P}\left(\boldsymbol{Y}_{1}=\left(x_{1}^{\prime}, x_{2}^{\prime}, j^{\prime}\right) \mid \boldsymbol{Y}_{0}=\left(x_{1}, x_{2}, j\right)\right)$, is given in terms of $s_{0} \times s_{0}$ nonnegative block matrices

$$
A_{i, j}, i, j \in \mathbb{H}, \quad A_{i, j}^{(1)}, i \in \mathbb{H}, j \in \mathbb{H}_{+}, \quad A_{i, j}^{(2)}, i \in \mathbb{H}_{+}, j \in \mathbb{H}, \quad A_{i, j}^{(0)}, i, j \in \mathbb{H}_{+},
$$

as follows: for $\left(x_{1}, x_{2}\right),\left(x_{1}^{\prime}, x_{2}^{\prime}\right) \in \mathbb{Z}_{+}^{2}$,

$$
\left(p_{\left(x_{1}, x_{2}, j\right),\left(x_{1}^{\prime}, x_{2}^{\prime}, j^{\prime}\right)}, j, j^{\prime} \in S_{0}\right)= \begin{cases}A_{\Delta x_{1}, \Delta x_{2}}, & \text { if } x_{1} \neq 0, x_{2} \neq 0, \Delta x_{1}, \Delta x_{2} \in \mathbb{H}, \\ A_{\Delta x_{1}, \Delta x_{2},}^{(1)}, & \text { if } x_{1} \neq 0, x_{2}=0, \Delta x_{1} \in \mathbb{H}, \Delta x_{2} \in \mathbb{H}_{+}, \\ A_{\Delta x_{1}, \Delta x_{2}}^{(2)}, & \text { if } x_{1}=0, x_{2} \neq 0, \Delta x_{1} \in \mathbb{H}_{+}, \Delta x_{2} \in \mathbb{H}, \\ A_{\Delta x_{1}, \Delta x_{2},}^{(0)}, & \text { if } x_{1}=x_{2}=0, \Delta x_{1}, \Delta x_{2} \in \mathbb{H}_{+}, \\ O, & \text { otherwise, }\end{cases}
$$

where $\Delta x_{1}=x_{1}^{\prime}-x_{1}$ and $\Delta x_{2}=x_{2}^{\prime}-x_{2}$. The matrices $A_{*, *}, A_{*, *}^{(1)}, A_{*, *}^{(2)}$ and $A_{*, *}^{(0)}$ defined as

$$
A_{*, *}=\sum_{i, j \in \mathbb{H}} A_{i, j}, \quad A_{*, *}^{(1)}=\sum_{i \in \mathbb{H}, j \in \mathbb{H}_{+}} A_{i, j}^{(1)}, \quad A_{*, *}^{(2)}=\sum_{i \in \mathbb{H}_{+}, j \in \mathbb{H}} A_{i, j}^{(2)}, \quad A_{*, *}^{(0)}=\sum_{i, j \in \mathbb{H}_{+}} A_{i, j}^{(0)},
$$

are stochastic. The Markov chain $\left\{\boldsymbol{Y}_{n}\right\}$ is called a discrete-time two-dimensional quasi-birth-anddeath (2D-QBD) process in Ozawa [18], where $\left(X_{1, n}, X_{2, n}\right)$ is called the level and $J_{n}$ the phase.

Remark 2.1. The $2 D-Q B D$ process explained above is a simplified model of that introduced in Ozawa [18]. In order to make the structure of the vector generating function defined later simple, we adopt the simplified model. A general $2 D-Q B D$ process can be reduced to a simplified one with keeping the stationary distribution unchanged: see Miyazawa [16].

We assume the following condition throughout the paper.

Assumption 2.1. The Markov chain $\left\{\boldsymbol{Y}_{n}\right\}$ is irreducible and aperiodic.

We consider three kinds of Markov chain generated from $\left\{\boldsymbol{Y}_{n}\right\}$ by removing one or two boundaries and denote them by $\left\{\tilde{\boldsymbol{Y}}_{n}\right\}=\left\{\left(\tilde{X}_{1, n}, \tilde{X}_{2, n}, \tilde{J}_{n}\right)\right\},\left\{\tilde{\boldsymbol{Y}}_{n}^{(1)}\right\}=\left\{\left(\tilde{X}_{1, n}^{(1)}, \tilde{X}_{2, n}^{(1)}, \tilde{J}_{n}^{(1)}\right)\right\}$ and $\left\{\tilde{\boldsymbol{Y}}_{n}^{(2)}\right\}=$ $\left\{\left(\tilde{X}_{1, n}^{(2)}, \tilde{X}_{2, n}^{(2)}, \tilde{J}_{n}^{(2)}\right)\right\}$, respectively. $\left\{\tilde{\boldsymbol{Y}}_{n}\right\}$ is the Markov chain on the state space $\mathbb{Z}^{2} \times S_{0}$, generated by removing the boundaries on the $x_{1}$ and $x_{2}$-axes, and the transition probability matrix

$$
\tilde{P}=\left(\tilde{p}_{\left(x_{1}, x_{2}, j\right),\left(x_{1}^{\prime}, x_{2}^{\prime}, j^{\prime}\right)},\left(x_{1}, x_{2}, j\right),\left(x_{1}^{\prime}, x_{2}^{\prime}, j^{\prime}\right) \in \mathbb{Z}^{2} \times S_{0}\right)
$$

where $\tilde{p}_{\left(x_{1}, x_{2}, j\right),\left(x_{1}^{\prime}, x_{2}^{\prime}, j^{\prime}\right)}=\mathbb{P}\left(\tilde{\boldsymbol{Y}}_{1}=\left(x_{1}^{\prime}, x_{2}^{\prime}, j^{\prime}\right) \mid \tilde{\boldsymbol{Y}}_{0}=\left(x_{1}, x_{2}, j\right)\right)$ is given as

$$
\left(\tilde{p}_{\left(x_{1}, x_{2}, j\right),\left(x_{1}^{\prime}, x_{2}^{\prime}, j^{\prime}\right)}, j, j^{\prime} \in S_{0}\right)= \begin{cases}A_{\Delta x_{1}, \Delta x_{2}}, & \text { if } \Delta x_{1}, \Delta x_{2} \in \mathbb{H} \\ O, & \text { otherwise }\end{cases}
$$

where $\Delta x_{1}=x_{1}^{\prime}-x_{1}$ and $\Delta x_{2}=x_{2}^{\prime}-x_{2}$. The Markov chain $\left\{\tilde{\boldsymbol{Y}}_{n}\right\}$ is a two-dimensional skip-free random walk on $\mathbb{Z}^{2}$ whose transition probabilities are modulated depending on the phase state $J_{n}$. From the definition of $\left\{\tilde{\boldsymbol{Y}}_{n}\right\}$, we see that it is governed only by the block matrices $A_{k, l}, k, l \in \mathbb{H}$. Hence, we call the Markov chain $\left\{\tilde{\boldsymbol{Y}}_{n}\right\}$ a Markov chain generated by $\left\{A_{k, l}, k, l \in \mathbb{H}\right\}$. We adopt the following definition. 
Definition 2.1. We say that the set of block matrices, $\left\{A_{k, l}, k, l \in \mathbb{H}\right\}$, is irreducible (resp. aperiodic) if the Markov chain generated by the set of block matrices is irreducible (resp. aperiodic).

$\left\{\tilde{\boldsymbol{Y}}_{n}^{(1)}\right\}$ is the Markov chain on the state space $\mathbb{Z} \times \mathbb{Z}_{+} \times S_{0}$, generated by removing the boundary on the $x_{2}$-axes, and the transition probability matrix

$$
\tilde{P}^{(1)}=\left(\tilde{p}_{\left(x_{1}, x_{2}, j\right),\left(x_{1}^{\prime}, x_{2}^{\prime}, j^{\prime}\right)}^{(1)},\left(x_{1}, x_{2}, j\right),\left(x_{1}^{\prime}, x_{2}^{\prime}, j^{\prime}\right) \in \mathbb{Z} \times \mathbb{Z}_{+} \times S_{0}\right),
$$

where $\tilde{p}_{\left(x_{1}, x_{2}, j\right),\left(x_{1}^{\prime}, x_{2}^{\prime}, j^{\prime}\right)}^{(1)}=\mathbb{P}\left(\tilde{\boldsymbol{Y}}_{1}^{(1)}=\left(x_{1}^{\prime}, x_{2}^{\prime}, j^{\prime}\right) \mid \tilde{\boldsymbol{Y}}_{0}^{(1)}=\left(x_{1}, x_{2}, j\right)\right)$ is given as

$$
\left(\tilde{p}_{\left(x_{1}, x_{2}, j\right),\left(x_{1}^{\prime}, x_{2}^{\prime}, j^{\prime}\right)}^{(1)}, j, j^{\prime} \in S_{0}\right)= \begin{cases}A_{\Delta x_{1}, \Delta x_{2},} & \text { if } x_{2} \neq 0, \Delta x_{1}, \Delta x_{2} \in \mathbb{H}, \\ A_{\Delta x_{1}, \Delta x_{2}}^{(1)}, & \text { if } x_{2}=0, \Delta x_{1} \in \mathbb{H}, \Delta x_{2} \in \mathbb{H}_{+}, \\ O, & \text { otherwise }\end{cases}
$$

where $\Delta x_{1}=x_{1}^{\prime}-x_{1}$ and $\Delta x_{2}=x_{2}^{\prime}-x_{2} .\left\{\tilde{\boldsymbol{Y}}_{n}^{(2)}\right\}$ is the Markov chain on the state space $\mathbb{Z}_{+} \times \mathbb{Z} \times S_{0}$, generated by removing the boundary on the $x_{1}$-axes, and the transition probability matrix $\tilde{P}^{(2)}$ is analogously given. $\left\{\tilde{\boldsymbol{Y}}_{n}^{(1)}\right\}$ is the Markov chain generated by $\left\{\left\{A_{k, l}, k, l \in \mathbb{H}\right\},\left\{A_{k, l}^{(1)}, k \in \mathbb{H}, j \in\right.\right.$ $\left.\left.\mathbb{H}_{+}\right\}\right\}$, and $\left\{\tilde{\boldsymbol{Y}}_{n}^{(2)}\right\}$ is that generated by $\left\{\left\{A_{k, l}, k, l \in \mathbb{H}\right\},\left\{A_{k, l}^{(2)}, k \in \mathbb{H}_{+}, j \in \mathbb{H}\right\}\right\}$. We also adopt the following definition.

Definition 2.2. We say that the set of block matrices, $\left\{\left\{A_{k, l}, k, l \in \mathbb{H}\right\},\left\{A_{k, l}^{(1)}, k \in \mathbb{H}, j \in \mathbb{H}_{+}\right\}\right\}$, is irreducible (resp. aperiodic) if the Markov chain generated by the set of block matrices is irreducible (resp. aperiodic). Irreducibleness and aperiodicity of $\left\{\left\{A_{k, l}, k, l \in \mathbb{H}\right\},\left\{A_{k, l}^{(2)}, k \in \mathbb{H}_{+}, j \in \mathbb{H}\right\}\right\}$ are analogously defined.

Hereafter, we assume the following condition throughout the paper.

Assumption 2.2. The sets of block matrices, $\left\{A_{k, l}, k, l \in \mathbb{H}\right\},\left\{\left\{A_{k, l}, k, l \in \mathbb{H}\right\},\left\{A_{k, l}^{(1)}, k \in \mathbb{H}, j \in\right.\right.$ $\left.\left.\mathbb{H}_{+}\right\}\right\}$and $\left\{\left\{A_{k, l}, k, l \in \mathbb{H}\right\},\left\{A_{k, l}^{(2)}, k \in \mathbb{H}_{+}, j \in \mathbb{H}\right\}\right\}$ are irreducible and aperiodic.

Since $A_{*, *}$ is the transition probability matrix of the background process $\left\{\tilde{J}_{n}\right\}$ of $\left\{\tilde{\boldsymbol{Y}}_{n}\right\}$, we immediately obtain the following proposition.

Proposition 2.1. Under Assumption 2.2, $A_{*, *}$ is irreducible and aperiodic.

$A_{*, *}$ is, therefore, positive recurrent and ergodic since its dimension is finite. We denote by $\boldsymbol{\pi}_{*, *}$ the stationary distribution of $A_{*, *}$.

\subsection{Stationary condition}

According to Ozawa [19], we state the condition on which the 2d-QBD process is positive recurrent. Before doing it, we define so-called induced Markov chains and the mean drift vectors derived from the induced Markov chains (see Fayolle et al. 2]). Since the 2D-QBD process is a kind of twodimensional random walk, there exist three induced Markov chains: $\mathcal{L}^{\{1,2\}}, \mathcal{L}^{\{1\}}$ and $\mathcal{L}^{\{2\}} . \mathcal{L}^{\{1,2\}}$ is the phase process $\left\{\tilde{J}_{n}\right\}$ of $\left\{\tilde{\boldsymbol{Y}}_{n}\right\}$ and it is a Markov chain governed by the transition probability matrix $A_{*, *}$. The mean drift vector $\boldsymbol{a}^{\{1,2\}}=\left(a_{1}^{\{1,2\}}, a_{2}^{\{1,2\}}\right)$ derived from $\mathcal{L}^{\{1,2\}}$ is given as

$$
a_{1}^{\{1,2\}}=\boldsymbol{\pi}_{*, *}\left(-A_{-1, *}+A_{1, *}\right) \mathbf{1}, \quad a_{2}^{\{1,2\}}=\boldsymbol{\pi}_{*, *}\left(-A_{*,-1}+A_{*, 1}\right) \mathbf{1}
$$


where for $k \in \mathbb{H}, A_{k, *}=\sum_{l \in \mathbb{H}} A_{k, l}$ and $A_{*, k}=\sum_{l \in \mathbb{H}} A_{l, k}$. Define block tri-diagonal transition probability matrices $A_{*}^{(1)}$ and $A_{*}^{(2)}$ as

$$
A_{*}^{(1)}=\left(\begin{array}{ccccc}
A_{*, 0}^{(1)} & A_{*, 1}^{(1)} & & & \\
A_{*,-1} & A_{*, 0} & A_{*, 1} & & \\
& A_{*,-1} & A_{*, 0} & A_{*, 1} & \\
& & \ddots & \ddots & \ddots
\end{array}\right), A_{*}^{(2)}=\left(\begin{array}{ccccc}
A_{0, *}^{(2)} & A_{1, *}^{(2)} & & & \\
A_{-1, *} & A_{0, *} & A_{1, *} & & \\
& A_{-1, *} & A_{0, *} & A_{1, *} & \\
& & \ddots & \ddots & \ddots
\end{array}\right)
$$

where for $k \in \mathbb{H}, A_{*, k}^{(1)}=\sum_{l \in \mathbb{H}} A_{l, k}^{(1)}$ and $A_{k, *}^{(2)}=\sum_{l \in \mathbb{H}} A_{k, l}^{(2)}$. We immediately obtain the following proposition.

Proposition 2.2. Under Assumption 2.2, $A_{*}^{(1)}$ and $A_{*}^{(2)}$ are irreducible and aperiodic.

$\mathcal{L}^{\{1\}}$ (resp. $\left.\mathcal{L}^{\{2\}}\right)$ is a partial process $\left\{\left(\tilde{X}_{2, n}^{(1)}, \tilde{J}_{n}^{(1)}\right)\right\}$ of $\left\{\tilde{\boldsymbol{Y}}_{n}^{(1)}\right\}\left(\operatorname{resp} .\left\{\left(\tilde{X}_{1, n}^{(2)}, \tilde{J}_{n}^{(2)}\right)\right\}\right.$ of $\left.\left\{\tilde{\boldsymbol{Y}}_{n}^{(2)}\right\}\right)$ and it is a Markov chain governed by $A_{*}^{(1)}$ (resp. $A_{*}^{(2)}$ ). Since $\mathcal{L}^{\{1\}}$ (resp. $\mathcal{L}^{\{2\}}$ ) is one-dimensional QBD process, it is positive recurrent if and only if $a_{2}^{\{1,2\}}<0$ (resp. $a_{1}^{\{1,2\}}<0$ ). Denote by $\boldsymbol{\pi}_{*}^{(1)}=$ $\left(\boldsymbol{\pi}_{*, k}^{(1)}, k \in \mathbb{Z}_{+}\right)$and $\boldsymbol{\pi}_{*}^{(2)}=\left(\boldsymbol{\pi}_{*, k}^{(2)}, k \in \mathbb{Z}_{+}\right)$the stationary distributions of $\mathcal{L}^{\{1\}}$ and $\mathcal{L}^{\{2\}}$, respectively, if they exist. Then, the mean increment vectors $\boldsymbol{a}^{\{1\}}=\left(a_{1}^{\{1\}}, a_{2}^{\{1\}}\right)$ and $\boldsymbol{a}^{\{2\}}=\left(a_{1}^{\{2\}}, a_{2}^{\{2\}}\right)$ derived from $\mathcal{L}^{\{1\}}$ and $\mathcal{L}^{\{2\}}$ are given by

$$
\begin{aligned}
& a_{1}^{\{1\}}=\boldsymbol{\pi}_{*, 0}^{(1)}\left(-A_{-1,0}^{(1)}-A_{-1,1}^{(1)}+A_{1,0}^{(1)}+A_{1,1}^{(1)}\right) \mathbf{1}+\boldsymbol{\pi}_{*, 1}^{(1)}\left(I-R_{*}^{(1)}\right)^{-1}\left(-A_{-1, *}+A_{1, *}\right) \mathbf{1}, \\
& a_{2}^{\{1\}}=0, \quad a_{1}^{\{2\}}=0, \\
& a_{2}^{\{2\}}=\boldsymbol{\pi}_{*, 0}^{(2)}\left(-A_{0,-1}^{(2)}-A_{1,-1}^{(2)}+A_{0,1}^{(2)}+A_{1,1}^{(2)}\right) \mathbf{1}+\boldsymbol{\pi}_{*, 1}^{(2)}\left(I-R_{*}^{(2)}\right)^{-1}\left(-A_{*,-1}+A_{*, 1}\right) \mathbf{1},
\end{aligned}
$$

where $R_{*}^{(1)}$ and $R_{*}^{(2)}$ are the rate matrix of $\mathcal{L}^{\{1\}}$ and $\mathcal{L}^{\{2\}}$, respectively. The condition on which $\left\{\boldsymbol{Y}_{n}\right\}$ is positive recurrent or transient is given by the following lemma.

Lemma 2.1 (Theorem 3.4 of Ozawa [19]). When $a_{1}^{\{1,2\}}<0$ and $a_{2}^{\{1,2\}}<0$, the 2D-QBD process $\left\{\boldsymbol{Y}_{n}\right\}$ is positive recurrent if $a_{1}^{\{1\}}<0$ and $a_{2}^{\{2\}}<0$, and it is transient if either $a_{1}^{\{1\}}>0$ or $a_{2}^{\{2\}}>0$. When $a_{1}^{\{1,2\}}>0$ and $a_{2}^{\{1,2\}}<0$, the $2 D-Q B D$ process is positive recurrent if $a_{1}^{\{1\}}<0$, and it is transient if $a_{1}^{\{1\}}>0$. When $a_{1}^{\{1,2\}}<0$ and $a_{2}^{\{1,2\}}>0$, the $2 D-Q B D$ process is positive recurrent if $a_{2}^{\{2\}}<0$, and it is transient if $a_{2}^{\{2\}}>0$. When $a_{1}^{\{1,2\}}>0$ and $a_{2}^{\{1,2\}}>0$, the 2D-QBD process is transient.

In order for the 2D-QBD $\left\{\boldsymbol{Y}_{n}\right\}$ to be positive recurrent, we assume the following condition throughout the paper.

Assumption 2.3. If $a_{1}^{\{1,2\}}<0$ and $a_{2}^{\{1,2\}}<0$, then $a_{1}^{\{1\}}<0$ and $a_{2}^{\{2\}}<0$; if $a_{1}^{\{1,2\}}>0$ and $a_{2}^{\{1,2\}}<0$, then $a_{1}^{\{1\}}<0$; if $a_{1}^{\{1,2\}}<0$ and $a_{2}^{\{1,2\}}>0$, then $a_{2}^{\{2\}}<0$.

Denote by $\boldsymbol{\nu}=\left(\nu_{k, l, j},(k, l, j) \in \mathbb{Z}_{+}^{2} \times S_{0}\right)$ the stationary distribution of $\left\{\boldsymbol{Y}_{n}\right\}$. We also define $\boldsymbol{\nu}_{k, l}$ for $k, l \in \mathbb{Z}_{+}$as $\boldsymbol{\nu}_{k, l}=\left(\nu_{k, l, j}, j \in S_{0}\right)$. In terms of $\boldsymbol{\nu}_{k, l}$, $\boldsymbol{\nu}$ is represented as $\boldsymbol{\nu}=$ $\left(\boldsymbol{\nu}_{k, l},(k, l) \in \mathbb{Z}_{+}^{2}\right)$. 


\subsection{QBD process with a countable phase space}

A QBD process with a countable phase space is a skip-free one-dimensional process on $\mathbb{Z}_{+}$with a background process on a countable state space. The one-dimensional process is the level and the background process the phase. A 2D-QBD process $\left\{\boldsymbol{Y}_{n}\right\}=\left\{\left(X_{1, n}, X_{2, n}, J_{n}\right)\right\}$ can be represented as a QBD process with a countable phase space in two ways: One is $\left\{\boldsymbol{Y}_{n}^{(1)}\right\}=\left\{\left(X_{1, n},\left(X_{2, n}, J_{n}\right)\right)\right\}$, where $X_{1, n}$ is the level and $\left(X_{2, n}, J_{n}\right)$ the phase, and the other $\left\{\boldsymbol{Y}_{n}^{(2)}\right\}=\left\{\left(X_{2, n},\left(X_{1, n}, J_{n}\right)\right)\right\}$, where $X_{2, n}$ is the level and $\left(X_{1, n}, J_{n}\right)$ the phase. Let $P^{(1)}$ and $P^{(2)}$ be the transition probability matrices of $\left\{Y_{n}^{(1)}\right\}$ and $\left\{Y_{n}^{(2)}\right\}$, respectively. They are represented in block form as follows:

$$
P^{(i)}=\left(\begin{array}{ccccc}
B_{0}^{(i)} & B_{1}^{(i)} & & & \\
A_{-1}^{(i)} & A_{0}^{(i)} & A_{1}^{(i)} & & \\
& A_{-1}^{(i)} & A_{0}^{(i)} & A_{1}^{(i)} & \\
& & \ddots & \ddots & \ddots
\end{array}\right), i=1,2,
$$

where each block is given by, for $j=0,1$,

$$
B_{j}^{(1)}=\left(\begin{array}{ccccc}
A_{j, 0}^{(0)} & A_{j, 1}^{(0)} & & & \\
A_{j,-1}^{(2)} & A_{j, 0}^{(2)} & A_{j, 1}^{(2)} & & \\
& A_{j,-1}^{(2)} & A_{j, 0}^{(2)} & A_{j, 1}^{(2)} & \\
& & \ddots & \ddots & \ddots
\end{array}\right), B_{j}^{(2)}=\left(\begin{array}{ccccc}
A_{0, j}^{(0)} & A_{1, j}^{(0)} & & & \\
A_{-1, j}^{(1)} & A_{0, j}^{(1)} & A_{1, j}^{(1)} & & \\
& A_{-1, j}^{(1)} & A_{0, j}^{(1)} & A_{1, j}^{(1)} & \\
& & \ddots & \ddots & \ddots
\end{array}\right),
$$

and for $j=-1,0,1$,

$$
A_{j}^{(1)}=\left(\begin{array}{ccccc}
A_{j, 0}^{(1)} & A_{j, 1}^{(1)} & & & \\
A_{j,-1} & A_{j, 0} & A_{j, 1} & & \\
& A_{j,-1} & A_{j, 0} & A_{j, 1} & \\
& & \ddots & \ddots & \ddots
\end{array}\right), A_{j}^{(2)}=\left(\begin{array}{ccccc}
A_{0, j}^{(2)} & A_{1, j}^{(2)} & & & \\
A_{-1, j} & A_{0, j} & A_{1, j} & & \\
& A_{-1, j} & A_{0, j} & A_{1, j} & \\
& & \ddots & \ddots & \ddots
\end{array}\right) .
$$

The transition probability matrices $A_{*}^{(1)}$ and $A_{*}^{(2)}$ defined in the previous subsection are represented as $A_{*}^{(1)}=\sum_{k \in \mathbb{H}} A_{k}^{(1)}$ and $A_{*}^{(2)}=\sum_{k \in \mathbb{H}} A_{k}^{(2)}$, respectively. Hence, $A_{*}^{(1)}$ (resp. $A_{*}^{(2)}$ ) is also the transition probability matrix of the phase process of $\left\{\boldsymbol{Y}_{n}^{(1)}\right\}$ (resp. $\left\{\boldsymbol{Y}_{n}^{(2)}\right\}$ ) when the level is greater than zero.

Denote by $\boldsymbol{\nu}^{(1)}=\left(\boldsymbol{\nu}_{n}^{(1)}, n \in \mathbb{Z}_{+}\right)$the stationary distribution of $\left\{\boldsymbol{Y}^{(1)}\right\}$ and by $\boldsymbol{\nu}^{(2)}=\left(\boldsymbol{\nu}_{n}^{(2)}, n \in\right.$ $\left.\mathbb{Z}_{+}\right)$that of $\left\{\boldsymbol{Y}^{(2)}\right\}$, where $\boldsymbol{\nu}_{n}^{(1)}=\left(\boldsymbol{\nu}_{n, k}, k \in \mathbb{Z}_{+}\right)$and $\boldsymbol{\nu}_{n}^{(2)}=\left(\boldsymbol{\nu}_{k, n}, k \in \mathbb{Z}_{+}\right)$. It is well known that each of $\boldsymbol{\nu}^{(1)}$ and $\boldsymbol{\nu}^{(2)}$ has a matrix geometric form (see References [17, 21], especially, Tweedie [21] for the case of QBD process with a countable phase space). Let $R^{(1)}$ and $R^{(2)}$ be the rate matrices of $\left\{\boldsymbol{Y}^{(1)}\right\}$ and $\left\{\boldsymbol{Y}^{(2)}\right\}$, respectively. They are given by the minimal nonnegative solutions to the matrix quadratic equations:

$$
R^{(1)}=\left(R^{(1)}\right)^{2} A_{-1}^{(1)}+R^{(1)} A_{0}^{(1)}+A_{1}^{(1)}, \quad R^{(2)}=\left(R^{(2)}\right)^{2} A_{-1}^{(2)}+R^{(2)} A_{0}^{(2)}+A_{1}^{(2)} .
$$

The matrix geometric forms of $\boldsymbol{\nu}^{(1)}$ and $\boldsymbol{\nu}^{(2)}$ are given by

$$
\boldsymbol{\nu}_{n}^{(1)}=\boldsymbol{\nu}_{1}^{(1)}\left(R^{(1)}\right)^{n-1}, \quad \boldsymbol{\nu}_{n}^{(2)}=\boldsymbol{\nu}_{1}^{(2)}\left(R^{(2)}\right)^{n-1}, n \geq 1 .
$$


Since $\boldsymbol{\nu}_{n}^{(1)}$ and $\boldsymbol{\nu}_{n}^{(2)}$ are represented as $\boldsymbol{\nu}_{n}^{(1)}=\left(\boldsymbol{\nu}_{n, k, j}, k \in \mathbb{Z}_{+}, j \in S_{0}\right)$ and $\boldsymbol{\nu}_{n}^{(2)}=\left(\boldsymbol{\nu}_{k, n, j}, k \in \mathbb{Z}_{+}, j \in\right.$ $S_{0}$ ), our aim corresponds to clarifying the asymptotic properties of the stationary distributions $\boldsymbol{\nu}_{n}^{(1)}$ and $\boldsymbol{\nu}_{n}^{(2)}$ as $n$ tends to infinity.

The triplet of block matrices $\left\{A_{-1}^{(1)}, A_{0}^{(1)}, A_{1}^{(1)}\right\}$ (resp. $\left\{A_{-1}^{(2)}, A_{0}^{(2)}, A_{1}^{(2)}\right\}$ ) is the Markov additive kernel of the Markov additive process obtained from $\left\{\boldsymbol{Y}_{n}^{(1)}\right\}$ (resp. $\left\{\boldsymbol{Y}_{n}^{(2)}\right\}$ ). Under Assumption 2.2. the Markov additive kernels $\left\{A_{-1}^{(1)}, A_{0}^{(1)}, A_{1}^{(1)}\right\}$ and $\left\{A_{-1}^{(2)}, A_{0}^{(2)}, A_{1}^{(2)}\right\}$ are 1-arithmetic, where the kernel is said to be 1-arithmetic if for some $k \geq 0$ and $j \in S_{0}$, the greatest common divisor of $\left\{n_{1}+\cdots+n_{l} ;\left[A_{n_{1}}^{(i)} A_{n_{2}}^{(i)} \cdots A_{n_{l}}^{(i)}\right]_{(k, j),(k, j)}>0, n_{1}, \ldots, n_{l} \in \mathbb{H}, l \geq 1\right\}$ is one (see, for example, Miyazawa and Zhao [13]). Since the Markov additive kernels are 1-arithmetic, $R^{(1)}$ and $R^{(2)}$ are aperiodic (see Remark 4.4 of Miyazawa and Zhao [13]). According to Ozawa [18, we assume the following condition throughout the paper.

Assumption 2.4. The rate matrices $R^{(1)}$ and $R^{(2)}$ are irreducible.

\subsection{Directional decay rates of the stationary distribution}

Here we summarize the results of Ozawa [18. Let $z_{1}$ and $z_{2}$ be positive numbers. Then, $C\left(z_{1}, z_{2}\right)$ is nonnegative and, by Assumption 2.2, it is irreducible and aperiodic. Let $\chi\left(z_{1}, z_{2}\right)$ be the PerronFrobenius eigenvalue of $C\left(z_{1}, z_{2}\right)$, i.e., $\chi\left(z_{1}, z_{2}\right)=\operatorname{spr}\left(C\left(z_{1}, z_{2}\right)\right)$. The modulus of every eigenvalue of $C\left(z_{1}, z_{2}\right)$ except $\chi\left(z_{1}, z_{2}\right)$ is strictly less than $\operatorname{spr}\left(C\left(z_{1}, z_{2}\right)\right)$. According to Kingman [7], we say that a positive function $f(x, y)$ is superconvex in $(x, y)$ if $\log f(x, y)$ is convex in $(x, y)$; a superconvex function is also a convex function. We have the following proposition.

Proposition 2.3 (Proposition 3.1 of Ozawa [18]). $\chi\left(e^{s_{1}}, e^{s_{2}}\right)$ is superconvex (hence convex) in $\left(s_{1}, s_{2}\right) \in \mathbb{R}^{2}$.

Define a closed set $\bar{\Gamma}$ as

$$
\bar{\Gamma}=\left\{\left(s_{1}, s_{2}\right) \in \mathbb{R}^{2}: \chi\left(e^{s_{1}}, e^{s_{2}}\right) \leq 1\right\} .
$$

Since $\chi(1,1)=\chi\left(e^{0}, e^{0}\right)=1, \bar{\Gamma}$ contains the point of $(0,0)$ and thus it is not empty. By Proposition 2.3, $\bar{\Gamma}$ is a convex set. The following property holds for $\bar{\Gamma}$.

Lemma 2.2. Under Assumption 2.2, $\bar{\Gamma}$ is bounded.

We give the proof of the lemma in Appendix $\mathrm{A}$. For $i \in\{1,2\}$, define the lower and upper extreme values of $\bar{\Gamma}$ with respect to $s_{i}$, denoted by $\underline{s}_{i}^{*}$ and $\bar{s}_{i}^{*}$, as

$$
\underline{s}_{i}^{*}=\min _{\left(s_{1}, s_{2}\right) \in \bar{\Gamma}} s_{i}, \quad \bar{s}_{i}^{*}=\max _{\left(s_{1}, s_{2}\right) \in \bar{\Gamma}} s_{i}
$$

where $-\infty<\underline{s}_{i}^{*} \leq 0$ and $0 \leq \bar{s}_{i}^{*}<\infty$. For $i \in\{1,2\}$, define $\underline{z}_{i}^{*}$ and $\bar{z}_{i}^{*}$ as $\underline{z}_{i}^{*}=e^{\underline{s}_{i}^{*}}$ and $\bar{z}_{i}^{*}=e^{\bar{s}_{i}^{*}}$, respectively, where $0<\underline{z}_{i}^{*} \leq 1$ and $1 \leq \bar{z}_{i}^{*}<\infty$. Since $\bar{\Gamma} \subset \mathbb{R}^{2}$ is a bounded convex set and $\chi\left(z_{1}, e^{s_{2}}\right)$ is convex in $s_{2} \in \mathbb{R}$, we see that, for each $z_{1} \in\left(\underline{z}_{1}^{*}, \bar{z}_{1}^{*}\right)$, equation $\chi\left(z_{1}, e^{s_{2}}\right)=1$ has just two real solutions in $s_{2} \in \mathbb{R}$. Furthermore, we also see that, for $z_{1}=\underline{z}_{1}^{*}$ or $\bar{z}_{1}^{*}$, equation $\chi\left(z_{1}, e^{s_{2}}\right)=1$ has just one real solution in $s_{2} \in \mathbb{R}$. This analogously holds for $\chi\left(e^{s_{1}}, z_{2}\right)$. Hence, we immediately obtain the following proposition (see Proposition 3.9 of Ozawa [18]). 
Proposition 2.4. For each $z_{1} \in\left(\underline{z}_{1}^{*}, \bar{z}_{1}^{*}\right)$ (resp. $z_{2} \in\left(\underline{z}_{2}^{*}, \bar{z}_{2}^{*}\right)$ ), equation $\chi\left(z_{1}, z_{2}\right)=1$ has just two different real solutions $\underline{\zeta}_{2}\left(z_{1}\right)$ and $\bar{\zeta}_{2}\left(z_{1}\right)$ (resp. $\underline{\zeta}_{1}\left(z_{2}\right)$ and $\bar{\zeta}_{1}\left(z_{2}\right)$ ), where $\underline{\zeta}_{2}\left(z_{1}\right)<\bar{\zeta}_{2}\left(z_{1}\right)$ (resp. $\left.\underline{\zeta}_{1}\left(z_{2}\right)<\bar{\zeta}_{1}\left(z_{2}\right)\right)$. For $z_{1}=\underline{z}_{1}^{*}$ or $\bar{z}_{1}^{*}$ (resp. $z_{2}=\underline{z}_{2}^{*}$ or $\left.\bar{z}_{2}^{*}\right)$, it has just one real solution $\underline{\zeta}_{2}\left(z_{1}\right)=\bar{\zeta}_{2}\left(z_{1}\right)$ (resp. $\underline{\zeta}_{1}\left(z_{2}\right)=\bar{\zeta}_{1}\left(z_{2}\right)$ ). If $z_{1} \notin\left[\underline{z}_{1}^{*}, \bar{z}_{1}^{*}\right]$ (resp. $z_{2} \notin\left[\underline{z}_{2}^{*}, \bar{z}_{2}^{*}\right]$ ), it has no real solutions.

Consider the following matrix quadratic equations of $X$ :

$$
\begin{aligned}
& A_{*,-1}(z)+A_{*, 0}(z) X+A_{*, 1}(z) X^{2}=X, \\
& A_{-1, *}(z)+A_{0, *}(z) X+A_{1, *}(z) X^{2}=X,
\end{aligned}
$$

where, for $i \in \mathbb{H}$,

$$
A_{*, i}(z)=\sum_{j \in \mathbb{H}} A_{j, i} z^{j}, \quad A_{i, *}(z)=\sum_{j \in \mathbb{H}} A_{i, j} z^{j} .
$$

Denote by $G_{1}(z)$ and $G_{2}(z)$ the minimum nonnegative solutions to matrix equations (2.4) and (2.5), respectively, if they exist. Furthermore, consider the following matrix quadratic equations of $X$ :

$$
\begin{aligned}
& X^{2} A_{*,-1}(z)+X A_{*, 0}(z)+A_{*, 1}(z)=X \\
& X^{2} A_{-1, *}(z)+X A_{0, *}(z)+A_{1, *}(z)=X .
\end{aligned}
$$

Denote by $R_{1}(z)$ and $R_{2}(z)$ the minimum nonnegative solutions to matrix equations (2.6) and (2.7), respectively, if they exist. Since $\bar{\Gamma}$ is convex, we see that, for every $s_{1} \in\left[\underline{s}_{1}^{*}, \bar{s}_{1}^{*}\right]$, there exists at least one real number $s_{2}$ satisfying $\chi\left(e^{s_{1}}, e^{s_{2}}\right) \leq 1$. This analogously holds for every $s_{2} \in\left[\underline{s}_{2}^{*}, \bar{s}_{2}^{*}\right]$. Hence, we obtain the next lemma.

Lemma 2.3 (Lemma 3.4 of Ozawa [18). For $z \in \mathbb{R}_{+} \backslash\{0\}$, the minimum nonnegative solutions $G_{1}(z)$ and $R_{1}(z)$ (resp. $G_{2}(z)$ and $R_{2}(z)$ ) to matrix equations (2.4) and (2.6) (resp. equations (2.5) and (2.7)) exist if and only if $z \in\left[\underline{z}_{1}^{*}, \bar{z}_{1}^{*}\right]$ (resp. $z \in\left[\underline{z}_{2}^{*}, \bar{z}_{2}^{*}\right]$ ).

$G_{1}(z), R_{1}(z), G_{2}(z)$ and $R_{2}(z)$ satisfy the following property.

Proposition 2.5 (Remark 3.5 of Ozawa [18]). For $z_{1} \in\left[\underline{z}_{1}^{*}, \bar{z}_{1}^{*}\right]$ and $z_{2} \in\left[\underline{z}_{2}^{*}, \bar{z}_{2}^{*}\right]$,

$$
\begin{array}{ll}
\operatorname{spr}\left(G_{1}\left(z_{1}\right)\right)=\underline{\zeta}_{2}\left(z_{1}\right), & \operatorname{spr}\left(R_{1}\left(z_{1}\right)\right)=\bar{\zeta}_{2}\left(z_{1}\right)^{-1}, \\
\operatorname{spr}\left(G_{2}\left(z_{2}\right)\right)=\underline{\zeta}_{1}\left(z_{2}\right), & \operatorname{spr}\left(R_{2}\left(z_{2}\right)\right)=\bar{\zeta}_{1}\left(z_{2}\right)^{-1} .
\end{array}
$$

Define matrix functions $C_{1}(z, X)$ and $C_{2}(X, z)$ as

$$
C_{1}(z, X)=A_{*, 0}^{(1)}(z)+A_{*, 1}^{(1)}(z) X, \quad C_{2}(X, z)=A_{0, *}^{(2)}(z)+A_{1, *}^{(2)}(z) X,
$$

where $X$ is an $s_{0} \times s_{0}$ matrix and, for $i \in \mathbb{H}_{+}$,

$$
A_{*, i}^{(1)}(z)=\sum_{j \in \mathbb{H}} A_{j, i}^{(1)} z^{j}, \quad A_{i, *}^{(2)}(z)=\sum_{j \in \mathbb{H}} A_{i, j}^{(2)} z^{j} .
$$

Define $\psi_{1}(z)$ and $\psi_{2}(z)$ as

$$
\psi_{1}(z)=\operatorname{spr}\left(C_{1}\left(z, G_{1}(z)\right)\right), \quad \psi_{2}(z)=\operatorname{spr}\left(C_{2}\left(G_{2}(z), z\right)\right) .
$$

By Propositions 3.5 and 3.6 of Ozawa [18] and their proofs, we obtain the following proposition. 
Proposition 2.6. For $i \in\{1,2\}, \psi_{i}\left(e^{s}\right)$ is superconvex (hence convex) in $s \in\left[\underline{s}_{i}^{*}, \bar{s}_{i}^{*}\right]$.

Next, we introduce points $\left(\theta_{1}^{(c)}, \theta_{2}^{(c)}\right)$ and $\left(\eta_{1}^{(c)}, \eta_{2}^{(c)}\right)$ on the closed curve $\chi\left(e^{s_{1}}, e^{s_{2}}\right)=1$, as follows:

$$
\begin{array}{ll}
\theta_{1}^{(c)}=\max \left\{s_{1} \in\left[\underline{s}_{1}^{*}, \bar{s}_{1}^{*}\right]: \psi_{1}\left(e^{s_{1}}\right) \leq 1\right\}, & \theta_{2}^{(c)}=\log \underline{\zeta}_{2}\left(e^{\theta_{1}^{(c)}}\right), \\
\eta_{2}^{(c)}=\max \left\{s_{2} \in\left[\underline{s}_{2}^{*}, \bar{s}_{2}^{*}\right]: \psi_{2}\left(e^{s_{2}}\right) \leq 1\right\}, & \eta_{1}^{(c)}=\log \underline{\zeta}_{1}\left(e^{\eta_{2}^{(c)}}\right) .
\end{array}
$$

Furthermore, define $\bar{\theta}_{2}^{(c)}$ and $\bar{\eta}_{1}^{(c)}$ as

$$
\bar{\theta}_{2}^{(c)}=\log \bar{\zeta}_{2}\left(e^{\theta_{1}^{(c)}}\right), \quad \bar{\eta}_{1}^{(c)}=\log \bar{\zeta}_{1}\left(e^{\eta_{2}^{(c)}}\right)
$$

Since $\bar{\Gamma}$ is a convex set, we can classify the possible configuration of points $\left(\theta_{1}^{(c)}, \theta_{2}^{(c)}\right)$ and $\left(\eta_{1}^{(c)}, \eta_{2}^{(c)}\right)$ in the following manner.

Type I: $\eta_{1}^{(c)}<\theta_{1}^{(c)}$ and $\theta_{2}^{(c)}<\eta_{2}^{(c)}, \quad$ Type II: $\eta_{1}^{(c)}<\theta_{1}^{(c)}$ and $\eta_{2}^{(c)} \leq \theta_{2}^{(c)}$,

Type III: $\theta_{1}^{(c)} \leq \eta_{1}^{(c)}$ and $\theta_{2}^{(c)}<\eta_{2}^{(c)}$.

Define the directional decay rates of the stationary distribution of the 2D-QBD process, denoted by $\xi_{1}$ and $\xi_{2}$, as

$$
\begin{array}{ll}
\xi_{1}=-\lim _{n \rightarrow \infty} \frac{1}{n} \log \nu_{n, j, k} & \text { for any } j \text { and } k, \\
\xi_{2}=-\lim _{n \rightarrow \infty} \frac{1}{n} \log \nu_{i, n, k} & \text { for any } i \text { and } k .
\end{array}
$$

Then, $\xi_{1}$ and $\xi_{2}$ are given as follows.

Lemma 2.4 (Corollary 4.3 of Ozawa [18]). Under Assumptions 2.1 to 2.4, the directional decay rates $\xi_{1}$ and $\xi_{2}$ exist and they are given by

$$
\left(\xi_{1}, \xi_{2}\right)= \begin{cases}\left(\theta_{1}^{(c)}, \eta_{2}^{(c)}\right), & \text { Tye I, } \\ \left(\bar{\eta}_{1}^{(c)}, \eta_{2}^{(c)}\right), & \text { Type II } \\ \left(\theta_{1}^{(c)}, \bar{\theta}_{2}^{(c)}\right), & \text { Type III. }\end{cases}
$$

Hence, under Assumptions 2.1 to 2.4, the directional geometric decay rate $r_{1}$ in $x_{1}$-coordinate and $r_{2}$ in $x_{2}$-coordinate exist, and they are given by $r_{1}=e^{\xi_{1}}$ and $r_{2}=e^{\xi_{2}}$.

\subsection{Main results}

We assume the following technical condition.

Assumption 2.5. All the eigenvalues of $G_{1}\left(r_{1}\right)$ are distinct. Also, those of $G_{2}\left(r_{2}\right)$ are distinct.

Remark 2.2. Assumption 2.5 is not necessary in Section [3. In Section 4, another assumption (Assumption 4.1) will be introduced instead of Assumption [2.5. Assumption 4.1 is a necessary condition for Assumption 2.5. In Section 5, we rely on Assumption 2.5. 
The exact asymptotic formula $h_{1}(k)$ of the stationary distribution in $x_{1}$-coordinate and $h_{2}(k)$ in $x_{2}$-coordinate, where

$$
\lim _{k \rightarrow \infty} \frac{\boldsymbol{\nu}_{k, 0}}{h_{1}(k)}=\text { nonzero constant vector }, \quad \lim _{k \rightarrow \infty} \frac{\boldsymbol{\nu}_{0, k}}{h_{2}(k)}=\text { nonzero constant vector },
$$

are given as follows.

Theorem 2.1 (Main results). Under Assumptions 2.1 through 2.5, in the case of Type I, the exact asymptotic formulae are given by

$$
h_{1}(k)=\left\{\begin{array}{ll}
r_{1}^{-k}, & \psi_{1}\left(\bar{z}_{1}^{*}\right)>1, \\
k^{-\frac{1}{2}\left(\bar{z}_{1}^{*}\right)^{-k},} & \psi_{1}\left(\bar{z}_{1}^{*}\right)=1, \\
k^{-\frac{1}{2}\left(2 l_{1}+1\right)}\left(\bar{z}_{1}^{*}\right)^{-k}, & \psi_{1}\left(\bar{z}_{1}^{*}\right)<1,
\end{array} \quad h_{2}(k)= \begin{cases}r_{2}^{-k}, & \psi_{2}\left(\bar{z}_{2}^{*}\right)>1, \\
k^{-\frac{1}{2}}\left(\bar{z}_{2}^{*}\right)^{-k}, & \psi_{2}\left(\bar{z}_{2}^{*}\right)=1, \\
k^{-\frac{1}{2}\left(2 l_{2}+1\right)}\left(\bar{z}_{2}^{*}\right)^{-k}, & \psi_{2}\left(\bar{z}_{2}^{*}\right)<1,\end{cases}\right.
$$

where $l_{1}$ and $l_{2}$ are some positive integers. In the case of Type II, they are given by

$$
h_{1}(k)=\left\{\begin{array}{ll}
r_{1}^{-k}, & \eta_{2}^{(c)}<\theta_{2}^{(c)}, \\
k r_{1}^{-k}, & \eta_{2}^{(c)}=\theta_{2}^{(c)} \text { and } \psi_{1}\left(\bar{z}_{1}^{*}\right)>1, \\
\left(\bar{z}_{1}^{*}\right)^{-k}, & \eta_{2}^{(c)}=\theta_{2}^{(c)} \text { and } \psi_{1}\left(\bar{z}_{1}^{*}\right)=1, \\
k^{-\frac{1}{2}}\left(\bar{z}_{1}^{*}\right)^{-k}, & \eta_{2}^{(c)}=\theta_{2}^{(c)} \text { and } \psi_{1}\left(\bar{z}_{1}^{*}\right)<1,
\end{array} \quad h_{2}(k)=r_{2}^{-k} .\right.
$$

In the case of Type III, the are given by

$$
h_{1}(k)=r_{1}^{-k}, \quad h_{2}(k)= \begin{cases}r_{2}^{-k}, & \theta_{1}^{(c)}<\eta_{1}^{(c)}, \\ k r_{2}^{-k}, & \theta_{1}^{(c)}=\eta_{1}^{(c)} \text { and } \psi_{2}\left(\bar{z}_{2}^{*}\right)>1, \\ \left(\bar{z}_{2}^{*}\right)^{-k}, & \theta_{1}^{(c)}=\eta_{1}^{(c)} \text { and } \psi_{2}\left(\bar{z}_{2}^{*}\right)=1, \\ k^{-\frac{1}{2}}\left(\bar{z}_{2}^{*}\right)^{-k}, & \theta_{1}^{(c)}=\eta_{1}^{(c)} \text { and } \psi_{2}\left(\bar{z}_{2}^{*}\right)<1 .\end{cases}
$$

This theorem will be proved in Section 5 .

\section{Generating functions and key expressions}

\subsection{Vector generating function}

Let $\varphi(z, w)$ be the vector generating function defined as

$$
\boldsymbol{\varphi}(z, w)=\sum_{i=0}^{\infty} \sum_{j=0}^{\infty} \boldsymbol{\nu}_{i, j} z^{i} w^{j}
$$

This vector generating function satisfies

$$
\boldsymbol{\varphi}(z, w)=\nu_{0,0}+\boldsymbol{\varphi}_{1}(z)+\boldsymbol{\varphi}_{2}(w)+\boldsymbol{\varphi}_{+}(z, w)
$$

where

$$
\boldsymbol{\varphi}_{1}(z)=\sum_{i=1}^{\infty} \boldsymbol{\nu}_{i, 0} z^{i}, \quad \boldsymbol{\varphi}_{2}(w)=\sum_{j=1}^{\infty} \boldsymbol{\nu}_{0, j} w^{j}, \quad \boldsymbol{\varphi}_{+}(z, w)=\sum_{i=1}^{\infty} \sum_{j=1}^{\infty} \boldsymbol{\nu}_{i, j} z^{i} w^{j}
$$


We define the following matrix functions:

$$
\begin{aligned}
& C(z, w)=\sum_{i \in \mathbb{H}} \sum_{j \in \mathbb{H}} A_{i, j} z^{i} w^{j}, \quad C_{0}(z, w)=\sum_{i \in \mathbb{H}_{+}} \sum_{j \in \mathbb{H}_{+}} A_{i, j}^{(0)} z^{i} w^{j}, \\
& C_{1}(z, w)=\sum_{i \in \mathbb{H}} \sum_{j \in \mathbb{H}_{+}} A_{i, j}^{(1)} z^{i} w^{j}, \quad C_{2}(z, w)=\sum_{i \in \mathbb{H}_{+}} \sum_{j \in \mathbb{H}} A_{i, j}^{(2)} z^{i} w^{j} .
\end{aligned}
$$

From the stationary equation $\boldsymbol{\nu}=\boldsymbol{\nu} P$, if $\boldsymbol{\varphi}(z, w)$ is finite, we obtain

$$
\begin{aligned}
& \boldsymbol{\varphi}(z, w) \\
& \quad=\boldsymbol{\nu}_{0,0} C_{0}(z, w)+\boldsymbol{\varphi}_{1}(z) C_{1}(z, w)+\boldsymbol{\varphi}_{2}(w) C_{2}(z, w)+\boldsymbol{\varphi}_{+}(z, w) C(z, w) .
\end{aligned}
$$

\subsection{Convergence domain}

Define the convergence domain $\mathcal{D}$ of the vector generating function $\varphi(z, w)$ as

$$
\mathcal{D}=\text { the interior of }\left\{(z, w) \in \mathbb{R}_{+}^{2}: \varphi(z, w)<\infty\right\} .
$$

We obtain a sufficiently large subset of the convergence domain $\mathcal{D}$. The radius of convergence of $\boldsymbol{\varphi}_{1}(z)$ and that of $\boldsymbol{\varphi}_{2}(z)$ are given in terms of $r_{1}$ and $r_{2}$, as follows.

Proposition 3.1. The radius of convergence of the vector generating function $\varphi_{1}(z)\left(r e s p . \varphi_{2}(z)\right)$ is given by $r_{1}$ (resp. $r_{2}$ ), i.e., for $i \in\{1,2\}$,

$$
\sup \left\{z \in \mathbb{R}_{+}: \varphi_{i}(z)<\infty\right\}=r_{i}
$$

Since this proposition is immediately obtained from Lemma 2.4 and Cauchy's criterion, we omit the proof.

Remark 3.1. From Proposition 3.1, we see that if $z>r_{1}$ or $w>r_{2}$, then at least one entry of $\boldsymbol{\varphi}(z, w)$ is divergent.

Define domains $\mathcal{D}_{0}$ and $\mathcal{D}_{1}$ as

$$
\begin{aligned}
& \mathcal{D}_{0}=\left\{(z, w) \in \mathbb{R}_{+}^{2}: 0<z<r_{1}, 0<w<r_{2}, \operatorname{spr}(C(z, w))<1\right\} \\
& \mathcal{D}_{1}=\left\{(z, w) \in \mathbb{R}_{+}^{2}: \text { there exists }\left(z_{1}^{\prime}, z_{2}^{\prime}\right) \in \mathcal{D}_{0} \text { such that }(z, w)<\left(z_{1}^{\prime}, z_{2}^{\prime}\right)\right\} .
\end{aligned}
$$

Then, we have the following lemma (see Kobayashi et al. [9]).

Lemma 3.1. $\mathcal{D}_{0} \subset \mathcal{D}_{1} \subset \mathcal{D}$.

This $\mathcal{D}_{1}$ is the desired subset of $\mathcal{D}$

Remark 3.2. $\mathcal{D}_{1}$ is probably identical to $\mathcal{D}$. This point is left as a further study. 


\subsection{Matrix-type vector generating functions and key expressions}

In order to investigate singularity of the generating functions $\varphi_{1}(z)$ and $\varphi_{2}(z)$, we need key expressions for $\varphi_{1}(z)$ and $\varphi_{2}(z)$ corresponding to equation (29) in Kobayashi and Miyazawa [8]. Therefore, we consider vector generating functions one of whose variables is a matrix. Here, we explain only about $\varphi_{1}(z)$ since results for $\varphi_{2}(z)$ are similar to those for $\varphi_{1}(z)$.

Define the following vector generating function:

$$
\boldsymbol{\varphi}(z, X)=\sum_{i=0}^{\infty} \sum_{j=0}^{\infty} \boldsymbol{\nu}_{i, j} z^{i} X^{j},
$$

where $z$ is a scalar and $X$ is an $s_{0} \times s_{0}$ matrix. We have

$$
\boldsymbol{\varphi}(z, X)=\varphi_{+}(z, X)+\varphi_{1}(z)+\varphi_{2}(X)+\nu_{0,0},
$$

where

$$
\boldsymbol{\varphi}_{+}(z, X)=\sum_{i=1}^{\infty} \sum_{j=1}^{\infty} \boldsymbol{\nu}_{i, j} z^{i} X^{j}, \quad \boldsymbol{\varphi}_{2}(X)=\sum_{j=1}^{\infty} \boldsymbol{\nu}_{0, j} X^{j} .
$$

If $\boldsymbol{\varphi}(z, X)<\infty$, we obtain from the stationary equation $\boldsymbol{\nu} P=\boldsymbol{\nu}$ that

$$
\begin{aligned}
\boldsymbol{\varphi}(z, X)=\sum_{i=1}^{\infty} & \sum_{j=1}^{\infty} \boldsymbol{\nu}_{i, j} z^{i} \hat{C}(z, X) X^{j-1}+\boldsymbol{\varphi}_{1}(z) C_{1}(z, X) \\
& +\sum_{j=1}^{\infty} \boldsymbol{\nu}_{0, j} \hat{C}_{2}(z, X) X^{j-1}+\boldsymbol{\nu}_{0,0} C_{0}(z, X),
\end{aligned}
$$

where

$$
\begin{array}{ll}
\hat{C}(z, X)=\sum_{j \in \mathbb{H}} A_{*, j}(z) X^{j+1}, & \hat{C}_{2}(z, X)=\sum_{j \in \mathbb{H}} A_{*, j}^{(2)}(z) X^{j+1}, \\
C_{0}(z, X)=\sum_{j \in \mathbb{H}_{+}} A_{*, j}^{(0)}(z) X^{j}, & C_{1}(z, X)=\sum_{j \in \mathbb{H}_{+}} A_{*, j}^{(1)}(z) X^{j},
\end{array}
$$

and for $j \in \mathbb{H}$ and $j^{\prime} \in \mathbb{H}_{+}$,

$$
\begin{aligned}
& A_{*, j}(z)=\sum_{i \in \mathbb{H}} A_{i, j} z^{i}, \quad A_{*, j}^{(2)}(z)=\sum_{i \in \mathbb{H}_{+}} A_{i, j}^{(2)} z^{i}, \quad A_{*, j^{\prime}}^{(1)}(z)=\sum_{i \in \mathbb{H}} A_{i, j}^{(1)} z^{i}, \\
& A_{*, j^{\prime}}^{(0)}(z)=\sum_{i \in \mathbb{H}_{+}} A_{i, j}^{(0)} z^{i} .
\end{aligned}
$$

Note that $C_{1}(z, X), A_{*, j}(z)$ and $A_{*, j}^{(1)}(z)$ have already been defined in Section 2, With respect to the convergence domain of $\varphi(z, X)$, we give the following lemma.

Lemma 3.2. For $n \in \mathbb{Z}_{+}$, let $\boldsymbol{a}_{n}$ be an $1 \times m$ complex vector and define a vector series $\phi(w)$ as $\phi(w)=\sum_{n=0}^{\infty} \boldsymbol{a}_{n} w^{n}$. Furthermore, for an $m \times m$ complex matrix $X$, define $\phi(X)$ as $\phi(X)=$ $\sum_{n=0}^{\infty} \boldsymbol{a}_{n} X^{n}$. Assume that $\phi(w)$ converges absolutely for all $w \in \mathbb{C}$ such that $|w|<r$ for some $r>0$. Then, if $\operatorname{spr}(X)<r, \phi(X)$ also converges absolutely. 
Since the proof of this lemma is elementary, we give it in Appendix B. By Lemma 3.1, $\varphi(z, w)$ converges absolutely for every $(z, w) \in \mathbb{C}^{2}$ such that $(|z|,|w|) \in \mathcal{D}_{1}$. Hence, by Lemma 3.2, we immediately obtain a criterion for convergence of $\varphi(z, X)$, as follows.

Proposition 3.2. Let $z$ be a complex number and $X$ an $s_{0} \times s_{0}$ complex matrix. Then, $\varphi(z, X)$ converges absolutely if $(|z|, \operatorname{spr}(X)) \in \mathcal{D}_{1}$.

The desired key expression for $\varphi_{1}(z)$ is given as follows.

Lemma 3.3. For $z \in\left[\underline{z}_{1}^{*}, \bar{z}_{1}^{*}\right]$, if $\left(z, \underline{\zeta}_{2}(z)\right) \in \mathcal{D}_{1}$, then we have

$$
\boldsymbol{\varphi}_{1}(z)=\left\{\boldsymbol{\varphi}_{2}^{\hat{C}_{2}}\left(z, G_{1}(z)\right)-\boldsymbol{\varphi}_{2}\left(G_{1}(z)\right)+\boldsymbol{\nu}_{0,0}\left(C_{0}\left(z, G_{1}(z)\right)-I\right)\right\}\left(I-C_{1}\left(z, G_{1}(z)\right)\right)^{-1},
$$

where

$$
\boldsymbol{\varphi}_{2}^{\hat{C}_{2}}(z, X)=\sum_{j=1}^{\infty} \boldsymbol{\nu}_{0, j} \hat{C}_{2}(z, X) X^{j-1}
$$

Proof. By Lemma 2.3 , for $z \in\left[\underline{z}_{1}^{*}, \bar{z}_{1}^{*}\right]$, the nonnegative matrix $G_{1}(z)$ exists. Since

$$
\left(z, \operatorname{spr}\left(G_{1}(z)\right)=\left(z, \underline{\zeta}_{2}(z)\right) \in \mathcal{D}_{1},\right.
$$

we have, by Proposition 3.2, $\varphi\left(z, G_{1}(z)\right)<\infty$. $G_{1}(z)$ satisfies equation (2.4), which corresponds to $\hat{C}(z, X)=X$, and we obtain, from equation (3.4),

$$
\boldsymbol{\varphi}\left(z, G_{1}(z)\right)=\boldsymbol{\varphi}_{+}\left(z, G_{1}(z)\right)+\boldsymbol{\varphi}_{1}(z) C_{1}\left(z, G_{1}(z)\right)+\boldsymbol{\varphi}_{2}^{\hat{C}_{2}}\left(z, G_{1}(z)\right)+\boldsymbol{\nu}_{0,0} C_{0}\left(z, G_{1}(z)\right) .
$$

Hence, from this equation and equation (3.3), we obtain

$$
\boldsymbol{\varphi}_{1}(z)\left(I-C_{1}\left(z, G_{1}(z)\right)\right)=\boldsymbol{\varphi}_{2}^{\hat{C}_{2}}\left(z, G_{1}(z)\right)-\boldsymbol{\varphi}_{2}\left(G_{1}(z)\right)+\boldsymbol{\nu}_{0,0}\left(C_{0}\left(z, G_{1}(z)\right)-I\right) .
$$

If $\left(z, \underline{\zeta}_{2}(z)\right) \in \mathcal{D}_{1}$, then $z<r_{1}$ and we have $\psi_{1}(z)=\operatorname{spr}\left(C_{1}\left(z, G_{1}(z)\right)<1\right.$. Hence, $I-C_{1}\left(z, G_{1}(z)\right)$ is nonsingular and we obtain expression (3.5).

A similar expression holds for $\varphi_{2}(z)$ and it is given as follows.

Proposition 3.3. For $w \in\left[\underline{z}_{2}^{*}, \bar{z}_{2}^{*}\right]$, if $\left(\underline{\zeta}_{1}(w), w\right) \in \mathcal{D}_{1}$, then we have

$$
\boldsymbol{\varphi}_{2}(w)=\left\{\boldsymbol{\varphi}_{1}^{\hat{C}_{1}}\left(G_{2}(w), w\right)-\boldsymbol{\varphi}_{1}\left(G_{2}(w)\right)+\boldsymbol{\nu}_{0,0}\left(C_{0}\left(G_{2}(w), w\right)-I\right)\right\}\left(I-C_{2}\left(G_{2}(w), w\right)\right)^{-1}
$$

where

$$
\boldsymbol{\varphi}_{1}^{\hat{C}_{1}}(X, w)=\sum_{i=1}^{\infty} \boldsymbol{\nu}_{i, 0} \hat{C}_{1}(X, w) X^{j-1} \quad \text { and } \quad \hat{C}_{1}(X, w)=\sum_{i \in \mathbb{H}} A_{i, *}^{(1)}(w) X^{j+1}
$$

$\boldsymbol{\varphi}_{1}(X), C_{0}(X, w)$ and $C_{2}(X, w)$ are defined in a manner similar to that used for defining $\varphi_{2}(X)$, $C_{0}(z, X)$ and $C_{1}(z, X)$. 


\section{Analytic extension of the G-matrix}

In order to investigate singularities of $\varphi_{1}(z)$ by using expression (3.5), we must extend $G_{1}(z)$ to complex variable $z$ and clarify singularities of $G_{1}(z)$. We carry out it in two steps: first, $G_{1}(z)$ is redefined as a series of matrices, which converges absolutely in a certain annular domain and next, it is analytically continued via the matrix quadratic equation (2.4). Since $G_{2}(z)$ can analogously be extended, we explain only about $G_{1}(z)$ in this section.

\subsection{First definition of $G_{1}(z)$}

For $z \in \mathbb{C}$, we redefine $G_{1}(z)$ in a manner similar to that used for defining the so-called $G$-matrix of a QBD processes (see Neuts [17]). For the purpose, we use the following sets of index sequences: for $n \geq 1$ and for $m \geq 1$,

$$
\begin{aligned}
& \mathscr{I}_{n}=\left\{\boldsymbol{i}_{(n)} \in \mathbb{H}^{n}: \sum_{l=1}^{k} i_{l} \geq 0 \text { for } k \in\{1,2, \ldots, n-1\} \text { and } \sum_{l=1}^{n} i_{l}=0\right\}, \\
& \mathscr{I}_{D, m, n}=\left\{\boldsymbol{i}_{(n)} \in \mathbb{H}^{n}: \sum_{l=1}^{k} i_{l} \geq-m+1 \text { for } k \in\{1,2, \ldots, n-1\} \text { and } \sum_{l=1}^{n} i_{l}=-m\right\}, \\
& \mathscr{I}_{U, m, n}=\left\{\boldsymbol{i}_{(n)} \in \mathbb{H}^{n}: \sum_{l=1}^{k} i_{l} \geq 1 \text { for } k \in\{1,2, \ldots, n-1\} \text { and } \sum_{l=1}^{n} i_{l}=m\right\},
\end{aligned}
$$

where $\boldsymbol{i}_{(n)}=\left(i_{1}, i_{2}, \ldots, i_{n}\right)$. For $n \geq 1$, let $Q_{11}^{(n)}(z), D_{1}^{(n)}(z)$ and $U_{1}^{(n)}(z)$ be defined as

$$
\begin{aligned}
Q_{11}^{(n)}(z) & =\sum_{\boldsymbol{i}_{(n)} \in \mathscr{I}_{n}} A_{*, i_{1}}(z) A_{*, i_{2}}(z) \cdots A_{*, i_{n}}(z), \\
D_{1}^{(n)}(z) & =\sum_{\boldsymbol{i}_{(n)} \in \mathscr{I}_{D, 1, n}} A_{*, i_{1}}(z) A_{*, i_{2}}(z) \cdots A_{*, i_{n}}(z), \\
U_{1}^{(n)}(z) & =\sum_{\boldsymbol{i}_{(n)} \in \mathscr{I}_{U, 1, n}} A_{*, i_{1}}(z) A_{*, i_{2}}(z) \cdots A_{*, i_{n}}(z),
\end{aligned}
$$

and let $N_{1}(z), R_{1}(z)$ and $G_{1}(z)$ be defined as

$$
N_{1}(z)=\sum_{n=0}^{\infty} Q_{11}^{(n)}(z), \quad G_{1}(z)=\sum_{n=1}^{\infty} D_{1}^{(n)}(z), \quad R_{1}(z)=\sum_{n=1}^{\infty} U_{1}^{(n)}(z),
$$

where $Q_{11}^{(0)}(z)=I$. Let $Q(z)$ be defined as

$$
Q(z)=\left(\begin{array}{cccc}
A_{*, 0}(z) & A_{*, 1}(z) & & \\
A_{*,-1}(z) & A_{*, 0}(z) & A_{*, 1}(z) & \\
& A_{*,-1}(z) & A_{*, 0}(z) & A_{*, 1}(z) \\
& \ddots & \ddots & \ddots
\end{array}\right)
$$

and denote by $\tilde{Q}(z)$ the fundamental matrix of $Q(z)$, i.e., $\tilde{Q}(z)=\sum_{n=0}^{\infty} Q(z)^{n}$. Then, for $n \geq 0$, $Q_{11}^{(n)}$ is the $(1,1)$-block of $Q(z)^{n}$ and $N_{1}(z)$ is that of $\tilde{Q}(z)$. When $z=1$, the $(i, j)$-entry of $D_{1}^{(n)}(1)$ 
is the probability that a QBD process starting in phase $i$ of level $l$ enters level $l-1$ for the first time just after $n$ steps and the phase at that time is $j$. Hence, $G_{1}(1)$ is the G-matrix of the QBD process in a usual sense. Furthermore, the $(i, j)$-entry of $U_{1}^{(n)}(1)$ is the probability that the QBD process starting in phase $i$ of level $l$ stays in phase $j$ of level $l+1$ just after $n$ steps and it does not enter any level lower than or equal to $l$ until that time. Hence, $R_{1}(1)$ is the rate matrix of the QBD process in a usual sense. $N_{1}(z), G_{1}(z)$ and $R_{1}(z)$ satisfy the following properties.

Lemma 4.1. For $z \in \mathbb{C}\left[\underline{z}_{1}^{*}, \bar{z}_{1}^{*}\right]$, the following statements hold.

(i) $N_{1}(z), G_{1}(z)$ and $R_{1}(z)$ converge absolutely and satisfy

$$
|N(z)| \leq N(|z|), \quad\left|G_{1}(z)\right| \leq G_{1}(|z|), \quad\left|R_{1}(z)\right| \leq R_{1}(|z|) .
$$

(ii) $G_{1}(z)$ and $R_{1}(z)$ are represented in terms of $N_{1}(z)$, as follows.

$$
G_{1}(z)=N_{1}(z) A_{*,-1}(z), \quad R_{1}(z)=A_{*, 1}(z) N_{1}(z) .
$$

(iii) $G_{1}(z)$ and $R_{1}(z)$ satisfy the following matrix quadratic equations:

$$
\begin{aligned}
& A_{*,-1}(z)+A_{*, 0}(z) G_{1}(z)+A_{*, 1}(z) G_{1}(z)^{2}=G_{1}(z), \\
& R_{1}(z)^{2} A_{*,-1}(z)+R_{1}(z) A_{*, 0}(z)+A_{*, 1}(z)=R_{1}(z) .
\end{aligned}
$$

(iv) Define $H_{1}(z)$ as $H_{1}(z)=A_{*, 0}(z)+A_{*, 1}(z) N_{1}(z) A_{*,-1}(z)$, then $N_{1}(z)$ satisfies

$$
\left(I-H_{1}(z)\right) N_{1}(z)=I \text {. }
$$

(v) For nonzero $w \in \mathbb{C}, I-C(z, w)$ satisfy the following factorization (e.g., see Lemma 3.1 of Miyazawa and Zhao [13]).

$$
I-C(z, w)=\left(w^{-1} I-R_{1}(z)\right)\left(I-H_{1}(z)\right)\left(w I-G_{1}(z)\right) .
$$

Since the proof of this lemma is elementary, we give it in Appendix C. From statement (iv) of Lemma 4.1, we have

$$
\operatorname{det}\left(I-H_{1}(z)\right) \operatorname{det} N_{1}(z)=1
$$

and this leads us to the following fact.

Corollary 4.1. For $z \in \mathbb{C}\left[\underline{z}_{1}^{*}, \bar{z}_{1}^{*}\right]$, both $I-H_{1}(z)$ and $N_{1}(z)$ are nonsingular and they satisfy $N_{1}(z)=\left(I-H_{1}(z)\right)^{-1}$.

Remark 4.1. For $w \in \mathbb{C}\left[\underline{z}_{2}^{*}, \bar{z}_{2}^{*}\right], R_{2}(w)$ and $G_{2}(w)$ can analogously be redefined and they satisfy

$$
I-C(z, w)=\left(z^{-1} I-R_{2}(w)\right)\left(I-H_{2}(w)\right)\left(z I-G_{2}(w)\right),
$$

where $H_{2}(w)=A_{0, *}(w)+A_{1, *}(w) G_{2}(w)$. Furthermore, $I-H_{2}(w)$ is nonsingular and $N_{2}(w)$ corresponding to $N_{1}(z)$ is given by $N_{2}(w)=\left(I-H_{2}(z)\right)^{-1}$. We will use these facts later. 
From the definition of $D_{1}^{(n)}(z)$, it can be seen that each entry of $D_{1}^{(n)}(z)$ is a Laurent polynomial in $z$ and hence, we can represent $D_{1}^{(n)}(z)$ as

$$
D_{1}^{(n)}(z)=\sum_{k=-n}^{n} D_{1, k}^{(n)} z^{k}
$$

where each $D_{1, k}^{(n)}$ is a nonnegative square matrix. Note that $G_{1}(z)$ converges absolutely for $z \in$ $\mathbb{C}\left[\underline{z}_{1}^{*}, \bar{z}_{1}^{*}\right]$. Hence, using the representation of $D_{1}^{(n)}(z)$ above, we obtain

$$
G_{1}(z)=\sum_{n=1}^{\infty} \sum_{k=-n}^{n} D_{1, k}^{(n)} z^{k}=\sum_{k=-\infty}^{\infty} z^{k} \sum_{n=\max \{|k|, 1\}}^{\infty} D_{1, k}^{(n)} .
$$

This is a Laurent expansion of $G_{1}(z)$, and since $G_{1}(z)$ is absolutely convergent in $\mathbb{C}\left[\underline{z}_{1}^{*}, \bar{z}_{1}^{*}\right]$, we obtain the following lemma (see, for example, Section II.1 of Markushevich [12]).

Lemma 4.2. $G_{1}(z)$ is analytic in the open annular domain $\mathbb{C}\left(\underline{z}_{1}^{*}, \bar{z}_{1}^{*}\right)$.

\subsection{Eigenvalues of $G_{1}(z)$}

To obtain the Jordan canonical form of $G_{1}(z)$ in the following subsection, we identify the eigenvalues of $G_{1}(z)$ and clarify their properties. The Jordan canonical forms of usual G-matrices have extensively been studied in Gail et al. [3]. For $z, w \in \mathbb{C}$, define an $s_{0}$-dimensional square matrix function $L(z, w)$ as

$$
L(z, w)=z w(C(z, w)-I)=z A_{*,-1}(z)+z\left(A_{*, 0}(z)-I\right) w+z A_{*, 1}(z) w^{2},
$$

and denote by $\phi(z, w)$ the determinant of $L(z, w)$, i.e., $\phi(z, w)=\operatorname{det} L(z, w) . L(z, w)$ is a matrix polynomial in $w$ with degree 2 and every entry of $L(z, w)$ is a polynomial in $z$ and $w . \phi(z, w)$ is also a polynomial in $z$ and $w$. Denote by $m$ the degree of $\phi(z, w)$ in $w$, where $s_{0} \leq m \leq 2 s_{0} . \phi(z, w)$ is represented as

$$
\phi(z, w)=\sum_{k=0}^{m} p_{k}(z) w^{k},
$$

where for $k \in\{0,1, \ldots, m\}, p_{k}(z)$ is a polynomial in $z$ and $p_{m}(z)$ is not identically zero. For $z \in \mathbb{C}$ such that $p_{m}(z) \neq 0$, let $\alpha_{1}(z), \alpha_{2}(z), \ldots, \alpha_{m}(z)$ be the solutions to $\phi(z, w)=0$, counting multiplicities, i.e., $\phi\left(z, \alpha_{k}(z)\right)=0$ for $k \in\{1,2, \ldots, m\}$. For nonzero $z, w \in \mathbb{C}$, let $\lambda_{1}^{C}(z, w), \lambda_{2}^{C}(z, w)$, $\ldots, \lambda_{s_{0}}^{C}(z, w)$ be the eigenvalues of $C(z, w)$, counting multiplicities. Recall that, for $z \in\left(\underline{z}_{1}^{*}, \bar{z}_{1}^{*}\right)$, equation $\chi(z, w)=\operatorname{spr}(C(z, w))=1$ has two different real solutions $\underline{\zeta}_{2}(z)<\bar{\zeta}_{2}(z)$, and for $z=\underline{z}_{1}^{*}$

or $\bar{z}_{1}^{*}$, it has just one real solution $\underline{\zeta}_{2}(z)=\bar{\zeta}_{2}(z)$. For $z \in \mathbb{C}\left[\underline{z}_{1}^{*}, \bar{z}_{1}^{*}\right]$, let $\lambda_{1}^{G_{1}}(z), \lambda_{2}^{G_{1}}(z), \ldots, \lambda_{s_{0}}^{G_{1}}(z)$ be the eigenvalues of $G_{1}(z)$, counting multiplicities, and $\lambda_{1}^{R_{1}}(z), \lambda_{2}^{R_{1}}(z), \ldots, \lambda_{s_{0}}^{R_{1}}(z)$ those of $R_{1}(z)$. Then, we have the following lemma.

Lemma 4.3. Let $z$ be a number in $\mathbb{C}\left(\underline{z}_{1}^{*}, \bar{z}_{1}^{*}\right]$ and $m=m(z)$ be the degree of $\phi(z, w)$ in $w$.

(i) When $z \neq \bar{z}_{1}^{*}$, there exist a positive number $\varepsilon$ and just $s_{0}$ solutions to $\phi(z, w)=0$, say $\alpha_{1}(z), \alpha_{2}(z), \ldots, \alpha_{s_{0}}(z)$, counting multiplicities, such that $\left|\alpha_{l}(z)\right|<\underline{\zeta}_{2}(|z|)+\varepsilon$ for all $l \in$ $\left\{1,2, \ldots, s_{0}\right\}$. If $z$ is not a real number, $\varepsilon$ can be set at zero. Furthermore, the other $\left(m-s_{0}\right)$ solutions to $\phi(z, w)=0$, say $\alpha_{s_{0}+1}(z), \alpha_{s_{0}+2}(z), \ldots, \alpha_{m}(z)$, counting multiplicities, satisfy $\left|\alpha_{l}(z)\right| \geq \bar{\zeta}_{2}(|z|)$ for all $l \in\left\{s_{0}+1, s_{0}+2, \ldots, m\right\}$. 
(ii) When $z=\bar{z}_{1}^{*}$, assume that the multiplicity of the solution $\alpha_{s_{0}}(z)=\underline{\zeta}_{2}\left(\bar{z}_{1}^{*}\right)$ as a zero of $\phi(z, w)$ is two. Then, there exist just $s_{0}-1$ solutions to $\phi(z, w)=0$, say $\alpha_{1}(z), \alpha_{2}(z), \ldots, \alpha_{s_{0}-1}(z)$, counting multiplicities, such that $\left|\alpha_{l}(z)\right|<\underline{\zeta}_{2}\left(\bar{z}_{1}^{*}\right)$ for all $l \in\left\{1,2, \ldots, s_{0}-1\right\}$. Furthermore, there exists a solution to $\phi(z, w)=0$, say $\alpha_{s_{0}+1}(z)$, such that $\alpha_{s_{0}+1}(z)=\underline{\zeta}_{2}\left(\bar{z}_{1}^{*}\right)\left(=\bar{\zeta}_{2}\left(\bar{z}_{1}^{*}\right)\right)$. The other $\left(m-s_{0}-1\right)$ solutions to $\phi(z, w)=0$, say $\alpha_{s_{0}+2}(z), \alpha_{s_{0}+3}(z), \ldots, \alpha_{m}(z)$, counting multiplicities, satisfy $\left|\alpha_{l}(z)\right|>\bar{\zeta}_{2}\left(\left|\bar{z}_{1}^{*}\right|\right)$ for all $l \in\left\{s_{0}+2, s_{0}+3, \ldots, m\right\}$.

(iii) In both the cases (i) and (ii), the set of the first $s_{0}$ solutions corresponds to the set of the eigenvalues of $G(z)$, i.e.,

$$
\left\{\alpha_{1}(z), \alpha_{2}(z), \ldots, \alpha_{s_{0}}(z)\right\}=\left\{\lambda_{1}^{G_{1}}(z), \lambda_{2}^{G_{1}}(z), \ldots, \lambda_{s_{0}}^{G_{1}}(z)\right\},
$$

and the set of the other $\left(m-s_{0}\right)$ solutions to $\phi(z, w)=0$ corresponds to the set of the reciprocals of the nonzero eigenvalues of $R_{1}(z)$, i.e.,

$$
\left\{\alpha_{s_{0}+1}(z), \alpha_{s_{0}+2}(z), \ldots, \alpha_{m}(z)\right\}=\left\{\lambda^{-1}: \lambda \in\left\{\lambda_{1}^{R_{1}}(z), \lambda_{2}^{R_{1}}(z), \ldots, \lambda_{s_{0}}^{R_{1}}(z)\right\}, \lambda \neq 0\right\} .
$$

Before proving Lemma 4.3, we present several propositions.

Proposition 4.1. Under Assumption 2.2, for $z, w \in \mathbb{C}$ such that $z \neq 0$ and $w \neq 0$, if $|z| \neq z$ or $|w| \neq w$, then $\operatorname{spr}(C(z, w))<\operatorname{spr}(C(|z|,|w|)$.

Since the proof of this proposition is elementary, we give it in Appendix D.

Proposition 4.2. The following statements hold.

(i) If $z \in \mathbb{C}\left(\underline{z}_{1}^{*}, \bar{z}_{1}^{*}\right)$, then for every $l \in\left\{1,2, \ldots, s_{0}\right\},\left|\lambda_{l}^{G_{1}}(z)\right| \leq \underline{\zeta}_{2}(|z|)$ and $\left|\lambda_{l}^{R_{1}}(z)\right| \leq \bar{\zeta}_{2}(|z|)^{-1}$, where $\underline{\zeta}_{2}(|z|)<\bar{\zeta}_{2}(|z|)$.

(ii) If $|z|=\bar{z}_{1}^{*}$ and $z \neq \bar{z}_{1}^{*}$, then for every $l \in\left\{1,2, \ldots, s_{0}\right\},\left|\lambda_{l}^{G_{1}}(z)\right|<\underline{\zeta}_{2}\left(\bar{z}_{1}^{*}\right)$ and $\left|\lambda_{l}^{R_{1}}(z)\right|<$ $\bar{\zeta}_{2}\left(\bar{z}_{1}^{*}\right)^{-1}$, where $\underline{\zeta}_{2}\left(\bar{z}_{1}^{*}\right)=\bar{\zeta}_{2}\left(\bar{z}_{1}^{*}\right)$.

(iii) Consider the case where $z=\bar{z}_{1}^{*}$ and assume that $\lambda_{s_{0}}^{G_{1}}(z)=\underline{\zeta}_{2}\left(\bar{z}_{1}^{*}\right)$ and $\lambda_{s_{0}}^{R_{1}}(z)=\bar{\zeta}_{2}\left(\bar{z}_{1}^{*}\right)^{-1}$. Furthermore, assume that the algebraic multiplicity of $\lambda_{s_{0}}^{G_{1}}(z)$ and that of $\lambda_{s_{0}}^{R_{1}}(z)$ are one. Then, for every $l \in\left\{1,2, \ldots, s_{0}-1\right\},\left|\lambda_{l}^{G_{1}}(z)\right|<\underline{\zeta}_{2}\left(\bar{z}_{1}^{*}\right)$ and $\left|\lambda_{l}^{R_{1}}(z)\right|<\bar{\zeta}_{2}\left(\bar{z}_{1}^{*}\right)^{-1}$, where $\underline{\zeta}_{2}\left(\bar{z}_{1}^{*}\right)=\bar{\zeta}_{2}\left(\bar{z}_{1}^{*}\right)$.

Proof. By Lemma 4.1-(i), we have, for $z \in \mathbb{C}\left[\underline{z}^{*}, \bar{z}_{1}^{*}\right]$,

$$
\begin{aligned}
& \operatorname{spr}\left(G_{1}(z)\right) \leq \operatorname{spr}\left(\left|G_{1}(z)\right|\right) \leq \operatorname{spr}\left(G_{1}(|z|)\right)=\underline{\zeta}_{2}(|z|) \\
& \operatorname{spr}\left(R_{1}(z)\right) \leq \operatorname{spr}\left(\left|R_{1}(z)\right|\right) \leq \operatorname{spr}\left(R_{1}(|z|)\right)=\bar{\zeta}_{2}(|z|)^{-1} .
\end{aligned}
$$

From these formulae and the fact that if $z \in \mathbb{C}\left(\underline{z}^{*}, \bar{z}_{1}^{*}\right)$, then $\underline{\zeta}_{2}(|z|)<\bar{\zeta}_{2}(|z|)$, we obtain Statement (i).

By Lemma 4.1-(v), we have, for $z \in \mathbb{C}\left[\underline{z}_{1}^{*}, \bar{z}_{1}^{*}\right]$,

$$
\phi(z, w)=(-1)^{s_{0}} z^{s_{0}} w^{s_{0}} f_{H}(z) \operatorname{det}\left(w^{-1} I-R_{1}(z)\right) \operatorname{det}\left(w I-G_{1}(z)\right),
$$


where $f_{H}(z)=\operatorname{det}\left(I-H_{1}(z)\right)$ and, by Corollary 4.1, we see $f_{H}(z) \neq 0$. Let $m$ be the degree of the polynomial $\phi(z, w)$ in $w$. Then, $\phi(z, w)$ has just $m$ zeros, say $\alpha_{1}(z), \alpha_{2}(z), \ldots, \alpha_{m}(z)$, counting multiplicities, and we have

$$
\phi(z, w)=p_{m}(z) \prod_{k=1}^{m}\left(w-\alpha_{k}(z)\right)
$$

where $p_{m}(z) \neq 0$. The eigenvalues of $G_{1}(z)$ (resp. $\left.R_{1}(z)\right)$ are the zeros of the characteristic poly$\operatorname{nomial} \operatorname{det}\left(w I-G_{1}(z)\right)$ in $w\left(\operatorname{resp} \cdot \operatorname{det}\left(w^{-1} I-R_{1}(z)\right)\right.$ in $\left.w^{-1}\right)$, and we have

$$
\operatorname{det}\left(w I-G_{1}(z)\right)=\prod_{l=1}^{s_{0}}\left(w-\lambda_{l}^{G_{1}}(z)\right), \quad \operatorname{det}\left(w^{-1} I-R_{1}(z)\right)=\prod_{l=1}^{s_{0}}\left(w^{-1}-\lambda_{l}^{R_{1}}(z)\right) .
$$

Hence, we obtain

$$
p_{m}(z) \prod_{k=1}^{m}\left(w-\alpha_{k}(z)\right)=(-1)^{s_{0}} z^{s_{0}} f_{H}(z)\left(\prod_{l=1}^{s_{0}}\left(w-\lambda_{l}^{G_{1}}(z)\right)\right)\left(\prod_{l=1}^{s_{0}}\left(1-w \lambda_{l}^{R_{1}}(z)\right)\right) .
$$

Both sides of equation (4.15) are polynomials in $w$. Hence, the degree of the right hand side of the equation must be $m$, and $2 s_{0}-m$ eigenvalues of $R_{1}(z)$ must be zero. Therefore, without loss of generality, we assume that $\lambda_{l}^{R_{1}}(z)=0$ for $l \in\left\{m-s_{0}+1, m-s_{0}+2, \ldots, 2 s_{0}\right\}$. Then, for $l \in\left\{1,2, \ldots, m-s_{0}\right\}, \lambda_{l}^{R_{1}}(z) \neq 0$ and equation (4.15) becomes

$$
\begin{aligned}
\phi(z, w) & =p_{m}(z) \prod_{k=1}^{m}\left(w-\alpha_{k}(z)\right) \\
& =p_{m}(z)\left(\prod_{l=1}^{s_{0}}\left(w-\lambda_{l}^{G_{1}}(z)\right)\right)\left(\prod_{l=1}^{m-s_{0}}\left(w-\lambda_{l}^{R_{1}}(z)^{-1}\right)\right),
\end{aligned}
$$

where

$$
p_{m}(z)=(-1)^{s_{0}} z^{s_{0}} f_{H}(z)\left(\prod_{l=1}^{m-s_{0}}\left(-\lambda_{l}^{R_{1}}(z)\right)\right) \neq 0 .
$$

By Proposition 4.1, for $|z|=\bar{z}_{1}^{*}$ such that $z \neq \bar{z}_{1}^{*}$ and for $|w|=\underline{\zeta}_{2}\left(\bar{z}_{1}^{*}\right)$, we obtain

$$
\operatorname{spr}(C(z, w))<\operatorname{spr}\left(C\left(\bar{z}_{1}^{*}, \underline{\zeta}_{2}\left(\bar{z}_{1}^{*}\right)\right)=1 .\right.
$$

This implies that $\operatorname{det}(C(z, w)-I) \neq 0$ and $\phi(z, w)=z^{s_{0}} w^{s_{0}} \operatorname{det}(C(z, w)-I) \neq 0$. Hence, from equation (4.16), we see that this $w$ is neither an eigenvalue of $G_{1}(z)$ nor the reciprocal of a nonzero eigenvalue of $R_{1}(z)$. Therefore, from formulae (4.10) and (4.11), we obtain statement (ii).

Under the assumption of statement (iii), we consider the case where $z=\bar{z}_{1}^{*}$. Since the algebraic multiplicity of $\lambda_{s_{0}}^{G_{1}}(z)=\underline{\zeta}_{2}\left(\bar{z}_{1}^{*}\right)$ and that of $\lambda_{s_{0}}^{R_{1}}(z)=\bar{\zeta}_{2}\left(\bar{z}_{1}^{*}\right)^{-1}$ are one, we have, for every $l \in$ $\left\{1,2, \ldots, s_{0}-1\right\}, \lambda_{l}^{G_{1}}(z) \neq \underline{\zeta}_{2}\left(\bar{z}_{1}^{*}\right)$ and $\lambda_{l}^{R_{1}}(z) \neq \bar{\zeta}_{2}\left(\bar{z}_{1}^{*}\right)^{-1}$. Suppose that, for some $l \in\left\{1,2, \ldots, s_{0}-1\right\}$, $\left|\lambda_{l}^{G_{1}}(z)\right|=\underline{\zeta}_{2}\left(\bar{z}_{1}^{*}\right)$. Then, by Proposition 4 .1, we have $\phi\left(z, \lambda_{l}^{G_{1}}(z)\right) \neq 0$ and this contradicts equation (4.16). Hence, for every $l \in\left\{1,2, \ldots, s_{0}-1\right\},\left|\lambda_{l}^{G_{1}}(z)\right| \neq \underline{\zeta}_{2}\left(\bar{z}_{1}^{*}\right)$. Analogously, we have, for every $l \in\left\{1,2, \ldots, s_{0}-1\right\},\left|\lambda_{l}^{R_{1}}(z)\right| \neq \bar{\zeta}_{2}\left(\bar{z}_{1}^{*}\right)^{-1}$. Therefore, from formulae (4.10) and (4.11), we obtain statement (iii) and this completes the proof. 
Proof of Lemma 4.3. Since $p_{m}(z) \neq 0$, we obtain from equation (4.16) that

$$
\prod_{k=1}^{m}\left(w-\alpha_{k}(z)\right)=\left(\prod_{l=1}^{s_{0}}\left(w-\lambda_{l}^{G_{1}}(z)\right)\right)\left(\prod_{l=1}^{m-s_{0}}\left(w-\lambda_{l}^{R_{1}}(z)^{-1}\right)\right) .
$$

This equation implies that the eigenvalues of $G_{1}(z)$ and the reciprocals of the nonzero eigenvalues of $R_{1}(z)$ are the zeros of the polynomial $\phi(z, w)$ in $w$ and vice versa. Therefore, denoting the zeros of $\phi(z, w)$ corresponding to the eigenvalues of $G_{1}(z)$ by $\alpha_{1}(z), \alpha_{2}(z), \ldots, \alpha_{s_{0}}(z)$, we have

$$
\left\{\alpha_{1}(z), \alpha_{2}(z), \ldots, \alpha_{s_{0}}(z)\right\}=\left\{\lambda_{1}^{G_{1}}(z), \lambda_{2}^{G_{1}}(z), \ldots, \lambda_{s_{0}}^{G_{1}}(z)\right\} .
$$

Other zeros of $\phi(z, w), \alpha_{s_{0}+1}(z), \alpha_{s_{0}+2}(z), \ldots, \alpha_{m}(z)$, correspond to the reciprocals of the nonzero eigenvalues of $R_{1}(z)$. Hence, denoting them by $\lambda_{2 s_{0}-m+1}^{R_{1}}(z), \lambda_{2 s_{0}-m+2}^{R_{1}}(z), \ldots, \lambda_{s_{0}}^{R_{1}}(z)$, we have

$$
\begin{aligned}
\left\{\alpha_{s_{0}+1}(z), \alpha_{s_{0}+2}(z), \ldots, \alpha_{m}(z)\right\} & =\left\{\lambda^{-1}: \lambda \in\left\{\lambda_{1}^{R_{1}}(z), \lambda_{2}^{R_{1}}(z), \ldots, \lambda_{s_{0}}^{R_{1}}(z)\right\}, \lambda \neq 0\right\} \\
& =\left\{\lambda_{2 s_{0}-m+1}^{R_{1}}(z)^{-1}, \lambda_{2 s_{0}-m+2}^{R_{1}}(z)^{-1}, \ldots, \lambda_{s_{0}}^{R_{1}}(z)^{-1}\right\} .
\end{aligned}
$$

This complets the proof of statement (iii) of the Lemma.

If $|z| \neq \bar{z}_{1}^{*}$, then by Proposition 4.2 (i), setting $\varepsilon=\left(\bar{\zeta}_{2}(|z|)-\underline{\zeta}_{2}(|z|)\right) / 2$, we have, for every $l \in\left\{1,2, \ldots, s_{0}\right\}$ and $l^{\prime} \in\left\{2 s_{0}-m+1,2 s_{0}-m+2, \ldots, s_{0}\right\}$,

$$
\left|\lambda_{l}^{G_{1}}(z)\right|<\underline{\zeta}_{2}(|z|)+\varepsilon<\bar{\zeta}_{2}(|z|) \leq\left|\lambda_{l^{\prime}}^{R_{1}}(z)\right|^{-1} .
$$

Hence, from statement (iii) of the lemma, we obtain, for every $l \in\left\{1,2, \ldots, s_{0}\right\}$ and $l^{\prime} \in\left\{2 s_{0}-m+\right.$ $\left.1,2 s_{0}-m+2, \ldots, s_{0}\right\}$,

$$
\left|\alpha_{l}(z)\right|<\underline{\zeta}_{2}(|z|)+\varepsilon<\bar{\zeta}_{2}(|z|) \leq\left|\alpha_{l^{\prime}}(z)\right|^{-1} .
$$

When $|z| \neq z$, suppose $\left|\alpha_{l}(z)\right|=\underline{\zeta}_{2}(|z|)$ for some $l \in\left\{1,2, \ldots, s_{0}\right\}$. Then, by Proposition 4.1, we have $\operatorname{spr}\left(C\left(z, \alpha_{l}(z)\right)\right)<\operatorname{spr}\left(C\left(|z|, \zeta_{2}(|z|)\right)\right)=1$, and this contradicts $\phi\left(z, \alpha_{l}(z)\right)=0$. Hence, $\left|\alpha_{l}(z)\right| \neq \underline{\zeta}_{2}(|z|)$ and we see that $\varepsilon$ can be set at 0 when $|z| \neq z$. This completes the proof of statement (i) of the lemma.

If $|z|=\bar{z}_{1}^{*}$ and $z \neq \bar{z}_{1}^{*}$, then, by Proposition 4.2 (ii), setting

$$
\varepsilon=\frac{1}{2}\left(\min _{1 \leq k \leq m-s_{0}}\left|\lambda_{2 s_{0}-m+k}^{R_{1}}(z)\right|^{-1}-\bar{\zeta}_{2}\left(\bar{z}_{1}^{*}\right)\right),
$$

we have, for every $l \in\left\{1,2, \ldots, s_{0}\right\}$ and $l^{\prime} \in\left\{2 s_{0}-m+1,2 s_{0}-m+2, \ldots, s_{0}\right\}$,

$$
\left|\lambda_{l}^{G_{1}}(z)\right|<\bar{\zeta}_{2}\left(\bar{z}_{1}^{*}\right)+\varepsilon<\left|\lambda_{l^{\prime}}^{R_{1}}(z)\right|^{-1} .
$$

Under the assumption of statement (ii), if $z=\bar{z}_{1}^{*}$, we have just two solutions that equal $\underline{\zeta}_{2}\left(\bar{z}_{1}^{*}\right)$ : one is an eigenvalue of $G_{1}(z)$, say $\alpha_{s_{0}}(z)$, and the other that of $R_{1}(z)$, say $\alpha_{s_{0}+1}(z)$. The algebraic multiplicities of those eigenvalues are one. Hence, by Proposition 4.2 -(iii) and statement (iii) of the lemma, we obtain statement (ii) of the lemma. 


\subsection{Analytic continuation of $G_{1}(z)$}

Hereafter, we assume the following technical condition.

Assumption 4.1. For some $z_{0} \in \mathbb{C}\left(\underline{z}_{1}^{*}, \bar{z}_{1}^{*}\right]$, all the eigenvalues of $G_{1}\left(z_{0}\right)$ are distinct.

Although this assumption always holds under Assumption 2.5, we assume it in this section instead of Assumption 2.5] in order to make explanation simple. Under Assumption 4.1, we define a set of points at which the algebraic multiplicity of some eigenvalue of $G_{1}(z)$ is greater than one. Before doing it, we define a notation. Let $f(z, w)$ be an irreducible polynomial in $z$ and $w$ whose degree with respect to $w$ is $k$. Assume the degree $k$ is greater than or equal to one and denote by $a(z)$ the coefficient of $w^{k}$ in $f(z, w)$. This $a(z)$ is a polynomial in $z$ satisfying $a(z) \not \equiv 0$ but it may be a nonzero constant. Define a point set $\Xi(f)$ as

$$
\Xi(f)=\left\{z \in \mathbb{C}: a(z)=0 \text { or }\left(f(z, w)=0 \text { and } f_{w}(z, w)=0 \text { for some } w \in \mathbb{C}\right)\right\},
$$

where $f_{w}(z, w)=(\partial / \partial w) f(z, w)$. Each point in $\Xi(f)$ is called an exceptional point of the algebraic function $w=\alpha(z)$ defined by polynomial equation $f(z, w)=0$, and it is an algebraic singularity of $\alpha(z)$, i.e., a removable singularity, pole or branch point with a finite order. For any point $z \in \mathbb{C} \backslash \Xi(f), f(z, w)=0$ has just $k$ distinct solutions, which correspond to the $k$ branches of the algebraic function.

Without loss of generality, we assume that, for some $n \in \mathbb{N}$ and $l_{1}, l_{2}, \ldots, l_{n} \in \mathbb{N}$, the polynomial $\phi(z, w)=\operatorname{det} L(z, w)$ is factorized as

$$
\phi(z, w)=f_{1}(z, w)^{l_{1}} f_{2}(z, w)^{l_{2}} \cdots f_{n}(z, w)^{l_{n}},
$$

where $f_{k}(z, w), k=1,2, \ldots, n$, are irreducible polynomials in $z$ and $w$ and they are relatively prime. Here we note that, for some $k, f_{k}(z, w)$ may be a polynomial in either $z$ or $w$. Since the field of coefficients of polynomials we consider is $\mathbb{C}$, this factorization is unique. Let $z_{0}$ be the pint given in Assumption 4.1 and $\alpha_{k}\left(z_{0}\right), k=1,2, \ldots, s_{0}$, the solutions to $\phi\left(z_{0}, w\right)=0$ that correspond to the eigenvalues of $G_{1}\left(z_{0}\right)$. By Lemma 4.3, we see that, for every $k \in\left\{1,2, \ldots, s_{0}\right\}$, the solution $\alpha_{k}\left(z_{0}\right)$ is distinct from all other solutions to $\phi\left(z_{0}, w\right)=0$. Hence, for each $k \in\left\{1,2, \ldots, s_{0}\right\}$, there exists a unique $q(k) \in\{1,2, \ldots, n\}$ such that $\alpha_{k}\left(z_{0}\right)$ is a solution to $f_{q(k)}\left(z_{0}, w\right)=0$. Note that there may exist $k$ and $k^{\prime}$ such that $k \neq k^{\prime}$ and $q(k)=q\left(k^{\prime}\right)$. From factorization (4.19), we immediately know that $l_{q(k)}=1$ for $k \in\left\{1,2, \ldots, s_{0}\right\}$. Define a point set $\mathcal{E}_{1}$ as

$$
\mathcal{E}_{1}=\bigcup_{k=1}^{s_{0}} \Xi\left(f_{q(k)}\right) .
$$

Since, for each $k, f_{q(k)}$ is irreducible and not identically zero, the point set $\mathcal{E}_{1}$ is finite. If $z_{0} \in \mathcal{E}_{1}$, then replace $z_{0}$ with another point $z_{0}^{\prime}$ in a neighborhood of $z_{0}$ such that $z_{0}^{\prime} \in \mathbb{C}\left(\underline{z}_{1}^{*}, \bar{z}_{1}^{*}\right] \backslash \mathcal{E}_{1}$ and it satisfies the condition of Assumption 4.1. It is possible because of continuity of the solutions to $\phi\left(z_{0}, w\right)=0$ and finiteness of $\mathcal{E}_{1}$. Hereafter, in that case, we denote $z_{0}^{\prime}$ by $z_{0}$. Note that $\bar{z}_{1}^{*} \in \mathcal{E}_{1}$. This will be explained in Subsection 4.4 (see Lemma 4.6).

For every $k \in\left\{1,2, \ldots, s_{0}\right\}$ and any $z \in \mathbb{C}\left(\underline{z}_{1}^{*}, \bar{z}_{1}^{*}\right] \backslash \mathcal{E}_{1}$, let $\check{\alpha}_{k}(z)$ be the analytic continuation of $\alpha_{k}\left(z_{0}\right)$ along a common path on $\mathbb{C}\left(\underline{z}_{1}^{*}, \bar{z}_{1}^{*}\right] \backslash \mathcal{E}_{1}$, starting from $z_{0}$ and terminating at $z$. We give the following lemma. 
Lemma 4.4. For any $z \in \mathbb{C}\left(\underline{z}_{1}^{*}, \bar{z}_{1}^{*}\right] \backslash \mathcal{E}_{1}$, the set $\left\{\check{\alpha}_{1}(z), \check{\alpha}_{2}(z), \ldots, \check{\alpha}_{s_{0}}(z)\right\}$ is unique, i.e., it does not depend on the path along which all $\check{\alpha}_{k}(z), k=1,2, \ldots, s_{0}$, are simultaneously continued, and it is identical to the set of the eigenvalues of $G_{1}(z)$.

Proof of Lemma 4.4. Note that, for each $k \in\left\{1,2, \ldots, s_{0}\right\}, \check{\alpha}_{k}(z)$ is a branch of the algebraic function given by the polynomial $f_{q(k)}(z, w)$ and it can analytically be continued along any path on $\mathbb{C} \backslash \Xi\left(f_{q(k)}\right)$. Further note that $\bar{z}_{1}^{*} \in \mathcal{E}_{1}$ (see Subsection 4.4). Consider three real valued functions of a complex variable $z$ : $\left|\check{\alpha}_{k}(z)\right|, \underline{\zeta}_{2}(|z|)$ and $\bar{\zeta}_{2}(|z|)$. We have, for $z \in \mathbb{C}\left(\underline{z}_{1}^{*}, \bar{z}_{1}^{*}\right), \underline{\zeta}_{2}(|z|)<\bar{\zeta}_{2}(|z|)$ and, for $z$ such that $|z|=\bar{z}_{1}^{*}, \underline{\zeta}_{2}(|z|)=\bar{\zeta}_{2}(|z|)$. Furthermore, $\check{\alpha}_{k}(z)$ is a solution to $\phi(z, w)=0$ and, by Lemma 4.3, we know that, for $z \in \mathbb{C}\left(\underline{z}_{1}^{*}, \bar{z}_{1}^{*}\right) \backslash \mathcal{E}_{1}, \check{\alpha}_{k}(z)$ satisfies either $\left|\check{\alpha}_{k}(z)\right| \leq \underline{\zeta}_{2}(|z|)$ or $\left|\check{\alpha}_{k}(z)\right| \geq \bar{\zeta}_{2}(|z|)$ and, for $z \in \mathbb{C}$ such that $|z|=\bar{z}_{1}^{*}$ and $z \neq \bar{z}_{1}^{*}, \check{\alpha}_{k}(z)$ satisfies either $\left|\check{\alpha}_{k}(z)\right|<\underline{\zeta}_{2}\left(\bar{z}_{1}^{*}\right)$ or $\left|\check{\alpha}_{k}(z)\right| \geq \bar{\zeta}_{2}\left(\bar{z}_{1}^{*}\right)=\underline{\zeta}_{2}\left(\bar{z}_{1}^{*}\right)$.

Let $z_{s}$ and $z_{t}$ be points in $\in \mathbb{C}\left(\underline{z}_{1}^{*}, \bar{z}_{1}^{*}\right] \backslash \mathcal{E}_{1}$ and assume that if $\left|z_{s}\right| \neq \bar{z}_{1}^{*}$, then $\left|\check{\alpha}_{k}\left(z_{s}\right)\right| \leq$ $\underline{\zeta}_{2}\left(\left|z_{s}\right|\right), k=1,2, \ldots, s_{0}$, and otherwise, $\left|\check{\alpha}_{k}\left(z_{s}\right)\right|<\underline{\zeta}_{2}\left(\bar{z}_{1}^{*}\right), k=1,2, \ldots, s_{0}$. Consider an arbitrary path $\xi_{z_{s}, z_{t}}$ on $\mathbb{C}\left(\underline{z}_{1}^{*}, \bar{z}_{1}^{*}\right] \backslash \mathcal{E}_{1}$, starting from $z_{s}$ and terminating at $z_{t}$. For each $k \in\left\{1,2, \ldots, s_{0}\right\}$, analytically continue $\check{\alpha}_{k}\left(z_{s}\right)$ along the path $\xi_{z_{s}, z_{t}}$. Then, we obtain function $\check{\alpha}_{k}(z)$, which is analytic on $\xi_{z_{s}, z_{t}}$. We show that $\left|\check{\alpha}_{k}\left(z_{t}\right)\right| \leq \underline{\zeta}_{2}\left(\left|z_{t}\right|\right)$ when $\left|z_{t}\right| \neq \bar{z}_{1}^{*}$ and that $\left|\check{\alpha}_{k}\left(z_{t}\right)\right|<\underline{\zeta}_{2}\left(\bar{z}_{1}^{*}\right)$ when $\left|z_{t}\right|=\bar{z}_{1}^{*}$. Suppose it is not the case. Then, we have that $\underline{\zeta}_{2}\left(\left|z_{t}\right|\right)<\bar{\zeta}_{2}\left(\left|z_{t}\right|\right) \leq\left|\check{\alpha}_{k}\left(z_{t}\right)\right|$ when $\left|z_{t}\right| \neq \bar{z}_{1}^{*}$ or that $\underline{\zeta}_{2}\left(\bar{z}_{1}^{*}\right) \leq\left|\check{\alpha}_{k}\left(z_{t}\right)\right|$ when $\left|z_{t}\right|=\bar{z}_{1}^{*}$. In both the cases, since $\left|\check{\alpha}_{k}(z)\right|, \underline{\zeta}_{2}(|z|)$ and $\bar{\zeta}_{2}(|z|)$ are continuous on the path $\xi_{z_{s}, z_{t}}$, there exists a point $z^{\prime} \in \xi_{z_{s}, z_{t}}$ such that $\left|z^{\prime}\right| \neq \bar{z}_{1}^{*}$ and $\underline{\zeta}_{2}\left(\left|z^{\prime}\right|\right)<\left|\check{\alpha}_{k}\left(z^{\prime}\right)\right|<\bar{\zeta}_{2}\left(\left|z^{\prime}\right|\right)$ or that $\left|z^{\prime}\right|=\bar{z}_{1}^{*}$ and $\left|\check{\alpha}_{k}\left(z^{\prime}\right)\right|=\underline{\zeta}_{2}\left(\bar{z}_{1}^{*}\right)$. This contradicts Lemma 4.3 since $z^{\prime} \neq \bar{z}_{1}^{*}$ and $\check{\alpha}_{k}\left(z^{\prime}\right)$ is a solution to $\phi\left(z^{\prime}, w\right)=0$. Hence, we obtain the desired result.

For $z_{t} \in \mathbb{C}\left(\underline{z}_{1}^{*}, \bar{z}_{1}^{*}\right] \backslash \mathcal{E}_{1}$, consider an arbitrary path $\xi_{z_{0}, z_{t}}$ on $\mathbb{C}\left(\underline{z}_{1}^{*}, \bar{z}_{1}^{*}\right] \backslash \mathcal{E}_{1}$. From the fact obtained above, we see that, for every $k \in\left\{1,2, \ldots, s_{0}\right\}$, by analytically continuing $\alpha_{k}\left(z_{0}\right)$ along the path $\xi_{z_{0}, z_{t}}$, we obtain $\check{\alpha}_{k}\left(z_{t}\right)$ satisfying $\left|\check{\alpha}_{k}\left(z_{t}\right)\right| \leq \underline{\zeta}_{2}\left(\left|z_{t}\right|\right)$. Each $\check{\alpha}_{k}\left(z_{t}\right)$ is a solution to $\phi\left(z_{t}, w\right)=0$ and, by Lemma 4.3, the set $\left\{\check{\alpha}_{1}\left(z_{t}\right), \check{\alpha}_{2}\left(z_{t}\right), \ldots, \check{\alpha}_{s_{0}}\left(z_{t}\right)\right\}$ is identical to the set of the eigenvalues of $G_{1}\left(z_{t}\right)$. This also implies that the set $\left\{\check{\alpha}_{1}\left(z_{t}\right), \check{\alpha}_{2}\left(z_{t}\right), \ldots, \check{\alpha}_{s_{0}}\left(z_{t}\right)\right\}$ remains unchained as a set even if we use anther path connecting $z_{0}$ and $z_{t}$ for continuing each $\alpha_{k}\left(z_{0}\right), k=1,2, \ldots, s_{0}$. This completes the proof.

Remark 4.2. For some $k \in\left\{1,2, \ldots, s_{0}\right\}$, there may exist a branch point of $\check{\alpha}_{k}(z)$ in $\mathbb{C}\left(\underline{z}_{1}^{*}, \bar{z}_{1}^{*}\right]$. Lemma 4.4 asserts that even in that case, the set $\left\{\check{\alpha}_{1}(z), \check{\alpha}_{2}(z), \ldots, \check{\alpha}_{s_{0}}(z)\right\}$ takes a single value as a set, for any $z \in \mathbb{C}\left(\underline{z}_{1}^{*}, \bar{z}_{1}^{*}\right] \backslash \mathcal{E}_{1}$.

Remark 4.3. From the proof of Lemma 4.4, we see that, for every $k \in\left\{1,2, \ldots, s_{0}\right\}, \check{\alpha}_{k}(z)$ is bounded in a neighborhood of every point in $\mathcal{E}_{1} \cap \mathbb{C}\left(\underline{z}_{1}^{*}, \bar{z}_{1}^{*}\right)$ since, for every $z \in \mathbb{C}\left(\underline{z}_{1}^{*}, \bar{z}_{1}^{*}\right] \backslash \mathcal{E}_{1}$,

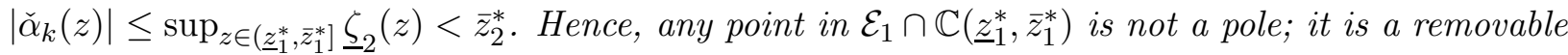
singularity or branch point.

Define a point set $\mathcal{E}_{2}$ as

$$
\mathcal{E}_{2}=\left\{z \in \mathbb{C} \backslash \mathcal{E}_{1}: f_{q(k)}(z, w)=f_{q\left(k^{\prime}\right)}(z, w)=0\right.
$$

for some $k, k^{\prime} \in\left\{1,2, \ldots, s_{0}\right\}$ such that $q(k) \neq q\left(k^{\prime}\right)$ and for some $\left.w \in \mathbb{C}\right\}$.

Since, for any $k, k^{\prime} \in\left\{1,2, \ldots, s_{0}\right\}$ such that $q(k) \neq q\left(k^{\prime}\right), f_{q(k)}(z, w)$ and $f_{q\left(k^{\prime}\right)}(z, w)$ are relatively prime, the point set $\mathcal{E}_{2}$ is finite. At each $z \in \mathcal{E}_{2} \cap \mathbb{C}\left(\underline{z}_{1}^{*}, \bar{z}_{1}^{*}\right]$, some eigenvalues of $G_{1}(z)$ may coalesce with each other but any point in $\mathcal{E}_{2}$ is not a singularity of any eigenvalue of $G_{1}(z)$. From Lemma 4.4, we immediately obtain the following. 
Corollary 4.2. For $z \in \mathbb{C}\left(\underline{z}_{1}^{*}, \bar{z}_{1}^{*}\right] \backslash\left(\mathcal{E}_{1} \cup \mathcal{E}_{2}\right)$, all the eigenvalues of $G_{1}(z), \alpha_{k}(z), k=1,2, \ldots, s_{0}$, are distinct. Furthermore, for each $z \in \mathbb{C}\left(\underline{z}_{1}^{*}, \bar{z}_{1}^{*}\right] \backslash\left(\mathcal{E}_{1} \cup \mathcal{E}_{2}\right)$ and each $k \in\left\{1,2, \ldots, s_{0}\right\}$, the multiplicity of $\alpha_{k}(z)$ as a zero of $L(z, w)$ is one.

Let $z_{0} \in \mathbb{C}\left(\underline{z}_{1}^{*}, \bar{z}_{1}^{*}\right] \backslash \mathcal{E}_{1}$ be the point given in Assumption 4.1 and, for $z_{t} \in \mathbb{C}\left(\underline{z}_{1}^{*}, \bar{z}_{1}^{*}\right] \backslash \mathcal{E}_{1}$, consider an arbitrary path $\xi_{z_{0}, z_{t}}$ on $\mathbb{C}\left(\underline{z}_{1}^{*}, \bar{z}_{1}^{*}\right] \backslash \mathcal{E}_{1}$. For $z$ in a neighborhood of each point on $\xi_{z_{0}, z_{t}}$, define a diagonal matrix function $\check{J}_{1}(z)$ as

$$
\check{J}_{1}(z)=\operatorname{diag}\left(\check{\alpha}_{1}(z), \check{\alpha}_{2}(z), \ldots, \check{\alpha}_{s_{0}}(z)\right),
$$

which is a Jordan canonical form of $G_{1}(z)$ on $\xi_{z_{0}, z_{t}} \backslash \mathcal{E}_{2}$. By Corollary 4.2 and the discussion in Section 7.1 of Gohberg et al. [4, if $z \in \xi_{z_{0}, z_{t}} \backslash \mathcal{E}_{2}$, then for every $k \in\left\{1,2, \ldots, s_{0}\right\}$, we have

$$
\operatorname{dim} \operatorname{Ker} L\left(z, \check{\alpha}_{k}(z)\right)=1
$$

and if $z \in \xi_{z_{0}, z_{t}} \cap \mathcal{E}_{2}$, then we have $\operatorname{dim} \operatorname{Ker} L\left(z, \check{\alpha}_{k}(z)\right) \geq 1$. Furthermore, $L\left(z, \check{\alpha}_{k}(z)\right)$ is entry-wise analytic on $\xi_{z_{0}, z_{t}}$. Hence, by Theorem S6.1 of Gohberg et al. [4, there exists a vector function $\breve{\boldsymbol{v}}_{k}(z)$ that is entry-wise analytic on $\xi_{z_{0}, z_{t}}$ and satisfies

$$
L\left(z, \check{\alpha}_{k}(z)\right) \check{\boldsymbol{v}}_{k}(z)=\mathbf{0}
$$

For each $z \in \xi_{z_{0}, z_{t}} \backslash \mathcal{E}_{2}, \check{\boldsymbol{v}}_{k}(z)$ is unique, up to multiplication by a constant, as a vector satisfying equation (4.20). Define a matrix function $\check{V}_{1}(z)$ as

$$
\check{V}_{1}(z)=\left(\begin{array}{llll}
\check{\boldsymbol{v}}_{1}(z) & \check{\boldsymbol{v}}_{2}(z) & \cdots & \check{\boldsymbol{v}}_{s_{0}}(z)
\end{array}\right) .
$$

In terms of $\check{J}_{1}(z)$ and $\check{V}_{1}(z)$, define a matrix function $\check{G}_{1}(z)$ as

$$
\check{G}_{1}(z)=\frac{\check{V}_{1}(z) \check{J}_{1}(z) \operatorname{adj} \check{V}_{1}(z)}{\operatorname{det} \check{V}_{1}(z)} .
$$

Since every entry of $\check{J}_{1}(z)$ and $\check{V}_{1}(z)$ is an analytic function on $\xi_{z_{0}, z_{t}}$, every entry of $\check{G}_{1}(z)$ is a meromorphic function on $\xi_{z_{0}, z_{t}}$. We give the following proposition.

Lemma 4.5. For any path $\xi_{z_{0}, z_{t}}$ on $\mathbb{C}\left(\underline{z}_{1}^{*}, \bar{z}_{1}^{*}\right] \backslash \mathcal{E}_{1}, \check{G}_{1}(z)$ is analytic and identical to $G_{1}(z)$ on $\xi_{z_{0}, z_{t}}$. Furthermore, for any $z \in \mathbb{C}\left(\underline{z}_{1}^{*}, \bar{z}_{1}^{*}\right] \backslash \mathcal{E}_{1}, \check{G}_{1}(z)$ is unique, i.e., it does not depend on the path along which $\check{G}_{1}(z)$ is continued.

Proof. First, we demonstrate that $\check{G}_{1}(z)$ is identical to $G_{1}(z)$ on $\xi_{z_{0}, z_{t}} \backslash \mathcal{E}_{2}$. By Lemma 4.3 and Corollary 4.1, both $\check{\alpha}_{k}(z)^{-1} I-R_{1}(z)$ and $I-H_{1}(z)$ are nonsingular for any $z \in \xi_{z_{0}, z_{t}}$ and $k \in$ $\left\{1,2, \ldots, s_{0}\right\}$, where $\bar{z}_{1}^{*} \notin \xi_{z_{0}, z_{t}}$. Hence, from equation (4.6), we obtain

$$
\check{\alpha}_{k}(z) I-G_{1}(z)=\left(I-H_{1}(z)\right)^{-1}\left(\check{\alpha}_{k}(z)^{-1} I-R_{1}(z)\right)^{-1}\left(I-C\left(z, \check{\alpha}_{k}(z)\right)\right) .
$$

For $k \in\left\{1,2, \ldots, s_{0}\right\}$, since $L\left(z, \check{\alpha}_{k}(z)\right)=z \check{\alpha}_{k}(z)\left(C\left(z, \check{\alpha}_{k}(z)\right)-I\right)$, equation (4.22) implies that $\check{\boldsymbol{v}}_{k}(z)$ is the eigenvector of $G_{1}(z)$ with respect to the eigenvalue $\check{\alpha}_{k}(z)$. For any $z \in \xi_{z_{0}, z_{t}} \backslash \mathcal{E}_{2}$, all the eigenvalues of $G_{1}(z)$ are distinct and the vectors $\check{\boldsymbol{v}}_{k}(z), k=1,2, \ldots, s_{0}$, are linearly independent. Hence, $\check{V}_{1}(z)$ is nonsingular and we have, for $z \in \xi_{z_{0}, z_{t}} \backslash \mathcal{E}_{2}$,

$$
G_{1}(z)=\check{V}_{1}(z) \check{J}_{1}(z) \check{V}_{1}(z)^{-1}=\check{G}_{1}(z)
$$


For any $z_{1} \in \xi_{z_{0}, z_{t}} \cap \mathcal{E}_{2}$, we have

$$
\lim _{\xi_{z_{0}, z_{t}} \ni z \rightarrow z_{1}} \check{G}_{1}(z)=\lim _{\xi_{z_{0}, z_{t}} \ni z \rightarrow z_{1}} G_{1}(z)=G_{1}\left(z_{0}\right) .
$$

This implies that the point $z_{1}$ is not a pole of any entry of $\check{G}_{1}(z)$, and $\check{G}_{1}(z)$ is entry-wise analytic at $z=z_{1}$ since it is entry-wise meromorphic on $\xi_{z_{0}, z_{t}}$. By Lemma 4.4. since $\check{G}_{1}(z)$ remains unchanged by any permutation of the eigenvalues and eigenvectors in expression (4.21), it does not depend on the path along which $\check{G}_{1}(z)$ is continued. This completes the proof.

By Lemma 4.5 and the identity theorem, we see that $\breve{G}_{1}(z)$ is the analytic extension of $G_{1}(z)$. Denoting the extension by the same notation $G_{1}(z)$, we immediately obtain the following corollary.

Corollary 4.3. The extended $G_{1}(z)$ is analytic on $\partial \Delta_{\bar{z}_{1}^{*}} \backslash \mathcal{E}_{1}$, where $\partial \Delta_{\bar{z}_{1}^{*}}=\partial \Delta\left(0, \bar{z}_{1}^{*}\right)$.

\subsection{Singularity of $G_{1}(z)$ on $\partial \Delta_{\bar{z}_{1}^{*}}$}

By Lemma 4.2, $G_{1}(z)$ is entry-wise analytic in $\mathbb{C}\left(\underline{z}_{1}^{*}, \bar{z}_{1}^{*}\right)$ and, by Corollary 4.3 , the extended $G_{1}(z)$ is entry-wise analytic on $\partial \Delta_{\bar{z}_{1}^{*}} \backslash \mathcal{E}_{1}$. In this subsection, we will demonstrate that the extended $G_{1}(z)$ is also entry-wise analytic on $\partial \Delta_{\bar{z}_{1}^{*}} \cap \mathcal{E}_{1}$ except for the point $\bar{z}_{1}^{*}$.

First, we consider property of the eigenvalues of $G_{1}(z)$ at $z=\bar{z}_{1}^{*}$. Let $\lambda_{1}^{C}(z, w), \lambda_{2}^{C}(z, w), \ldots$, $\lambda_{s 0}^{C}(z, w)$ be the eigenvalues of $C(z, w)$, counting multiplicity. Without loss of generality, we assume $\lambda_{s_{0}}^{C}(z, w)$ satisfies $\lambda_{s_{0}}^{C}\left(\bar{z}_{1}^{*}, \underline{\zeta}_{2}\left(\bar{z}_{1}^{*}\right)\right)=\chi\left(\bar{z}_{1}^{*}, \underline{\zeta}_{2}\left(\bar{z}_{1}^{*}\right)\right)=1$. Since $C(1,1)\left(=A_{*, *}\right)$ is irreducible and aperiodic, $C\left(\bar{z}_{1}^{*}, \underline{\zeta}_{2}\left(\bar{z}_{1}^{*}\right)\right)$ is also irreducible and aperiodic. Hence, the eigenvalue $\lambda_{s_{0}}^{C}\left(\bar{z}_{1}^{*}, \underline{\zeta}_{2}\left(\bar{z}_{1}^{*}\right)\right)$ is simple, i.e., the algebraic multiplicity is one, since it is the Perron-Frobenius eigenvalue of $C\left(\bar{z}_{1}^{*}, \underline{\zeta}_{2}\left(\bar{z}_{1}^{*}\right)\right)$. By the implicit function theorem, this implies that $\lambda_{s_{0}}^{C}(z, w)$ is analytic as a function of two complex variables in a neighborhood $\mathbb{U}^{2}$ of the point $\left(\bar{z}_{1}^{*}, \underline{\zeta}_{2}\left(\bar{z}_{1}^{*}\right)\right) . \lambda_{s_{0}}^{C}(z, w)$ is, therefore, the analytic extension of $\chi(z, w)$ in $\mathbb{U}^{2}$. Since $L(z, w)=z w(C(z, w)-I)$, the eigenvalues of $L(z, w)$ are given by $z w\left(\lambda_{k}^{C}(z, w)-1\right), k=1,2, \ldots, s_{0}$, and they are the solutions to the characteristic equation $\operatorname{det}(x I-L(z, w))=0$. Hence, we have

$$
z^{s_{0}} w^{s_{0}} \prod_{k=1}^{s_{0}}\left(\lambda_{k}^{C}(z, w)-1\right)=\operatorname{det} L(z, w)=\phi(z, w) .
$$

This implies that if $\lambda_{k}^{C}(z, h(z))=1$ for some $k$ and for some function $h(z)$, then $w=h(z)$ is a solution to the polynomial equation $\phi(z, w)=0$ and vice versa, where we consider only the case where $z \neq 0$ and $h(z) \neq 0$.

Define functions $g, g_{w}$ and $g_{w^{2}}$ as

$$
g(z, w)=\lambda_{s_{0}}^{C}(z, w)-1, \quad g_{w}(z, w)=\frac{\partial}{\partial w} g(z, w), \quad g_{w_{2}}(z, w)=\frac{\partial^{2}}{\partial w^{2}} g(z, w),
$$

where $g(z, w)$ is analytic in a neighborhood $\mathbb{U}^{2}$ of $\left(\bar{z}_{1}^{*}, \underline{\zeta}_{2}\left(\bar{z}_{1}^{*}\right)\right)$ and satisfies $g\left(\bar{z}_{1}^{*}, \underline{\zeta}_{2}\left(\bar{z}_{1}^{*}\right)\right)=0$. Since equation $\chi\left(\bar{z}_{1}^{*}, w\right)=1$ has the multiple real root $\underline{\zeta}_{2}\left(\bar{z}_{1}^{*}\right)=\bar{\zeta}_{2}\left(\bar{z}_{1}^{*}\right)$, we have $g_{w}\left(\bar{z}_{1}^{*}, \underline{\zeta}_{2}\left(\bar{z}_{1}^{*}\right)\right)=0$. Let the neighborhood $\mathbb{U}^{2}$ be so small that $g(z, w) \neq 0$ and $g_{w}(z, w) \neq 0$ in $\mathbb{U}^{2} \backslash\left\{\left(\bar{z}_{1}^{*}, \underline{\zeta}_{2}\left(\bar{z}_{1}^{*}\right)\right)\right\}$. It is possible since both $g(z, w)$ and $g_{w}(z, w)$ are analytic at $(z, w)=\left(\bar{z}_{1}^{*}, \zeta_{2}\left(\bar{z}_{1}^{*}\right)\right)$. By the implicit function theorem and the identity theorem, we, therefore, have a function $h(z)$ that is analytic and satisfies $g(z, h(z))=0$ for $z \in \mathbb{U}_{\bar{z}_{1}^{*}} \backslash\left\{\bar{z}_{1}^{*}\right\}$ and $h(z)=\underline{\zeta}_{2}(z)$ for $z \in\left(\underline{z}_{1}^{*}, \bar{z}_{1}^{*}\right) \cap \mathbb{U}_{\bar{z}_{1}^{*}}$, where $\mathbb{U}_{\bar{z}_{1}^{*}}$ is a 
neighborhood of the point $\bar{z}_{1}^{*}$. By the discussion above, $w=h(z)$ is a solution to $\phi(z, w)=0$ and hence we denote it by $\alpha_{s_{0}}(z)$. This $\alpha_{s_{0}}(z)$ is also an eigenvalue of $G_{1}(z)$, which is the maximum eigenvalue when $z \in\left(\underline{z}_{1}^{*}, \bar{z}_{1}^{*}\right] \cap \mathbb{U}_{\bar{z}_{1}^{*}}$. We give the following lemma.

Lemma 4.6. The point $\bar{z}_{1}^{*}$ is a branch point of $\alpha_{s_{0}}(z)$ with order one.

Proof. By Proposition $2.3, \chi\left(\bar{z}_{1}^{*}, e^{t}\right)$ is convex in $t \in \mathbb{R}$ and we obtain

$$
0 \neq\left.\frac{d^{2}}{d t^{2}} g\left(\bar{z}_{1}^{*}, e^{t}\right)\right|_{t=\log \underline{\zeta}_{2}\left(\bar{z}_{1}^{*}\right)}=\underline{\zeta}_{2}\left(\bar{z}_{1}^{*}\right)^{2} g_{w^{2}}\left(\bar{z}_{1}^{*}, \underline{\zeta}_{2}\left(\bar{z}_{1}^{*}\right)\right)
$$

where we use the fact that $g_{w}\left(\bar{z}_{1}^{*}, \zeta_{2}\left(\bar{z}_{1}^{*}\right)\right)=0$. This implies that $g_{w^{2}}\left(\bar{z}_{1}^{*}, \zeta_{2}\left(\bar{z}_{1}^{*}\right)\right) \neq 0$ and we know that the multiplicity of $\underline{\zeta}_{2}\left(\bar{z}_{1}^{*}\right)$ as a zero of $g\left(\bar{z}_{1}^{*}, w\right)$ is just two. We show that the multiplicity of $\underline{\zeta}_{2}\left(\bar{z}_{1}^{*}\right)$ as a zero of $\phi\left(\bar{z}_{1}^{*}, w\right)$ is also just two. Suppose it is not the case. Then, there are at least three identical solutions to $\phi\left(\bar{z}_{1}^{*}, w\right)=0$, including $\underline{\zeta}_{2}\left(\bar{z}_{1}^{*}\right)$ and $\bar{\zeta}_{2}\left(\bar{z}_{1}^{*}\right)$. Denote the other solution by $\alpha\left(\bar{z}_{1}^{*}\right)$, which equals $\underline{\zeta}_{2}\left(\bar{z}_{1}^{*}\right)$. Then, by equation (4.23), the solution must satisfies, for some $k \in\left\{1,2, \ldots, s_{0}-1\right\}, \lambda_{k}^{C}\left(\bar{z}_{1}^{*}, \alpha\left(\bar{z}_{1}^{*}\right)\right)=1$. This contradicts that the eigenvalue $\lambda_{s_{0}}^{C}\left(\bar{z}_{1}^{*}, \underline{\zeta}_{2}\left(\bar{z}_{1}^{*}\right)\right)$, which equals one, is simple, and we obtain the desired result. By the implicit function theorem, for $z$ in a neighborhood of $\bar{z}_{1}^{*}$ such that $z \neq \bar{z}_{1}^{*}$, we have

$$
\alpha_{s_{0}}^{\prime}(z)=-\frac{g_{z}\left(z, \alpha_{s_{0}}(z)\right)}{g_{w}\left(z, \alpha_{s_{0}}(z)\right)}
$$

where $\alpha_{s_{0}}^{\prime}(z)=(d / d z) \alpha_{s_{0}}(z)$ and $g_{z}(z, w)=(\partial / \partial z) g(z, w)$. Since $\chi\left(e^{s}, e^{t}\right)$ is convex in $(s, t) \in \mathbb{R}^{2}$, we have $g_{z}\left(\bar{z}_{1}^{*}, \underline{\zeta}_{2}\left(\bar{z}_{1}^{*}\right)\right) \neq 0$. Hence,

$$
\lim _{\mathbb{R} \ni z \rightarrow \bar{z}_{1-}^{*}}\left|\alpha_{s_{0}}^{\prime}(z)\right|=\infty
$$

and $\bar{z}_{1}^{*}$ is a singularity not being removable. Since $\alpha_{s_{0}}(z)$ is bounded in a neighborhood of $\bar{z}_{1}^{*}$, the point $\bar{z}_{1}^{*}$ is not a pole of $\alpha_{s_{0}}(z)$. Hence, it is a branch point of $\alpha_{s_{0}}(z)$. Since the multiplicity of $\alpha_{s_{0}}\left(\bar{z}_{1}^{*}\right)$ as a zero of $\phi\left(\bar{z}_{1}^{*}, w\right)$ is two, the order of the branch point is one.

Denote by $\alpha_{s_{0}+1}(z)$ the other branch that coalesces with $\alpha_{s_{0}}(z)$ at $z=\bar{z}_{1}^{*} \cdot \alpha_{s_{0}+1}(z)$ is a solution to $f_{q\left(s_{0}\right)}(z, w)=0$ and satisfies $\alpha_{s_{0}+1}(z)=\bar{\zeta}_{2}(z)$ for every $z \in\left(\underline{z}_{1}^{*}, \bar{z}_{1}^{*}\right] \cap \mathbb{U}_{\bar{z}_{1}^{*}}$, where $\mathbb{U}_{\bar{z}_{1}^{*}}$ is a neighborhood of $\bar{z}_{1}^{*}$. It is also the reciprocal of an eigenvalue of $R_{1}(z)$. From Lemma 4.6. it is expected that the point $\bar{z}_{1}^{*}$ is also a branch point of $G_{1}(z)$. We state this point in the next lemma. Before doing it, we define a matrix function $G_{1}^{c o}(z)$ that coalesces with $G_{1}(z)$ at $z=\bar{z}_{1}^{*}$, according to Lemma 4 of Li and Zhao [11]. We use the same notations in the previous subsection. Let $\boldsymbol{u}_{s_{0}}(z)$ be the left eigenvector of $G_{1}(z)$ with respect to the eigenvalue $\alpha_{s_{0}}(z)$. Let $\boldsymbol{v}_{s_{0}+1}(z)$ be the column vector function that satisfies $L\left(z, \alpha_{s_{0}+1}\right) \boldsymbol{v}_{s_{0}+1}(z)=\mathbf{0}$ and $\boldsymbol{u}_{s_{0}}(z) \boldsymbol{v}_{s_{0}+1}(z)=1$. Define $G_{1}^{c o}(z)$ as

$$
G_{1}^{c o}(z)=G_{1}(z)+\left(\alpha_{s_{0}+1}(z) I-G_{1}(z)\right) \boldsymbol{v}_{s_{0}+1}(z) \boldsymbol{u}_{s_{0}}(z) .
$$

Then, in a manner similar to that used in the proof of Lemma 4 of Li and Zhao [11], we see that $G_{1}^{c o}(z)$ satisfies the following matrix quadratic equation:

$$
A_{*,-1}(z)+\left(I-A_{*, 0}(z)\right) G_{1}^{c o}(z)+A_{*, 1}(z) G_{1}^{c o}(z)^{2}=O .
$$

The eigenvalues of $G_{1}^{c o}(z)$ are given by $\alpha_{1}(z), \alpha_{2}(z), \ldots, \alpha_{s_{0}-1}(z)$ and $\alpha_{s_{0}+1}(z)$, and the corresponding right eigenvectors are given by $\boldsymbol{v}_{1}(z), \boldsymbol{v}_{2}(z), \ldots, \boldsymbol{v}_{s_{0}-1}(z)$ and $\boldsymbol{v}_{s_{0}+1}(z)$, respectively. We present the following lemma. 
Lemma 4.7. $G_{1}(z)$ is analytic on the circle $\partial \Delta_{\bar{z}_{1}^{*}}$ except for $\bar{z}_{1}^{*}$. The point $\bar{z}_{1}^{*}$ is a branch pint of $G_{1}(z)$ with order one, and $G_{1}(z)$ coalesces with $G_{1}^{c o}(z)$ at the point.

Proof. Let $z_{1}$ be a point on $\partial \Delta_{\bar{z}_{1}^{*}} \cap \mathcal{E}_{1}$. First, assuming $\left(\partial \Delta_{\bar{z}_{1}^{*}} \backslash\left\{\bar{z}_{1}^{*}\right\}\right) \cap \mathcal{E}_{1} \neq \emptyset$, we consider the case where $z_{1} \neq \bar{z}_{1}^{*}$. Let $\mathbb{U}_{z_{1}}$ be a neighborhood of $z_{1}$ satisfying $\left(\mathbb{U}_{z_{1}} \backslash\left\{z_{1}\right\}\right) \cap \mathcal{E}_{1}=\emptyset$. We set $\mathbb{U}_{z_{1}}$ being so small that there exists a positive number $\varepsilon$ such that for every $z \in \mathbb{U}_{z_{1}},\left|\alpha_{k}(z)\right|<\underline{\zeta}_{2}\left(\bar{z}_{1}^{*}\right)-\varepsilon$ for $k \in\left\{1,2, \ldots, s_{0}\right\}$ and $\left|\alpha_{k}(z)\right| \geq \zeta_{2}\left(\bar{z}_{1}^{*}\right)-\varepsilon$ for $k \in\left\{s_{0}+1, s_{0}+2, \ldots, m\right\}$. By Lemma 4.3, it is possible since $\left|\alpha_{k}\left(z_{1}\right)\right|<\underline{\zeta}_{2}\left(\bar{z}_{1}^{*}\right)$ for $k \in\left\{1,2, \ldots, s_{0}\right\}$ and $\left|\alpha_{k}\left(z_{1}\right)\right| \geq \underline{\zeta}_{2}\left(\bar{z}_{1}^{*}\right)$ for $k \in\left\{s_{0}+1, s_{0}+2, \ldots, m\right\}$. For $k \in\left\{1,2, \ldots, s_{0}\right\}$ and for $z \in \mathbb{U}_{z_{1}}$, let $\boldsymbol{v}_{k}(z)$ be a vector function satisfies

$$
L\left(z, \alpha_{k}(z)\right) \boldsymbol{v}_{k}(z)=\mathbf{0} .
$$

Like $\check{\boldsymbol{v}}_{k}(z), \boldsymbol{v}_{k}(z)$ is unique, up to multiplication by a constant, and analytic in $\mathbb{U}_{z_{1}} \backslash\left\{z_{1}\right\}$. For $z \in \mathbb{U}_{z_{1}}$, define a matrix function $V_{1}(z)$ as

$$
V_{1}(z)=\left(\begin{array}{llll}
\boldsymbol{v}_{1}(z) & \boldsymbol{v}_{2}(z) & \cdots & \boldsymbol{v}_{s_{0}}(z)
\end{array}\right)
$$

Define a point set $\mathcal{E}_{3}\left(\mathbb{U}_{z_{1}}\right)$ as

$$
\mathcal{E}_{3}\left(\mathbb{U}_{z_{1}}\right)=\left\{z \in \mathbb{U}_{z_{1}} \backslash\left\{z_{1}\right\}: \operatorname{det} V_{1}(z)=0\right\} .
$$

Since $\operatorname{det} V_{1}(z)$ is analytic in $\mathbb{U}_{z_{1}} \backslash\left\{z_{1}\right\}$ and $\operatorname{det} V_{1}(z) \not \equiv 0$, we know by the identity theorem that there are no accumulation points of $\mathcal{E}_{3}\left(\mathbb{U}_{z_{1}}\right)$ in $\mathbb{U}_{z_{1}} \backslash\left\{z_{1}\right\}$. We show that $z_{1}$ is not an accumulation point of $\mathcal{E}_{3}\left(\mathbb{U}_{z_{1}}\right)$. Note that, by Lemma 4.3, for every $k \in\left\{1,2, \ldots, s_{0}\right\}, \alpha_{k}\left(z_{1}\right)$ is bounded and the point $z_{1}$ is a branch point or removable singularity of $\alpha_{k}(z)$. For $k \in\left\{1,2, \ldots, s_{0}\right\}$, if $z_{1}$ is a branch point of $\alpha_{k}(z)$, let $\nu_{k}$ be the order of the branch point; if $z_{1}$ is a removable singularity, let $\nu_{k}=0$. Furthermore, let $\nu$ be the least common multiple of $\left\{\nu_{1}+1, \nu_{2}+1, \ldots, \nu_{s_{0}}+1\right\}$. For $k \in\left\{1,2, \ldots, s_{0}\right\}$, define a function $\tilde{\alpha}_{k}(\zeta)$ analytic in a neighborhood of the origin, as follows: if $z_{1}$ is a branch point of $\alpha_{k}(z)$, let $\tilde{\alpha}_{k}(\zeta)$ be the function that is analytic in a neighborhood of the origin and satisfies

$$
\alpha_{k}(z)=\tilde{\alpha}_{k}\left(\left(z_{1}-z\right)^{\frac{1}{\nu_{k}+1}}\right)
$$

if $z_{1}$ is a removable singularity, define $\tilde{\alpha}_{k}(\zeta)$ as $\tilde{\alpha}_{k}(\zeta)=\alpha_{k}\left(z_{1}-\zeta\right)$. In the former case, considering the Puiseux series expansion of $\alpha_{k}(z)$ around $z_{1}$, it can be seen that such an analytic function exists. For $k \in\left\{1,2, \ldots, s_{0}\right\}$, define a vector function $\tilde{\boldsymbol{v}}_{k}(\zeta)$ entry-wise analytic in a neighborhood of the origin, as follows: if $z_{1}$ is a branch point of $\alpha_{k}(z)$, let $\tilde{\boldsymbol{v}}_{k}(\zeta)$ be the vector function that is entry-wise analytic in a neighborhood of the origin and satisfies

$$
L\left(z_{1}-\zeta^{\nu_{k}+1}, \tilde{\alpha}_{k}(\zeta)\right) \tilde{\boldsymbol{v}}_{k}(\zeta)=\mathbf{0}
$$

if $z_{1}$ is a removable singularity, define $\tilde{\boldsymbol{v}}_{k}(\zeta)$ as $\tilde{\boldsymbol{v}}_{k}(\zeta)=\boldsymbol{v}_{k}\left(z_{1}-\zeta\right)$. In the former case, since every entry of $L\left(z_{1}-\zeta^{\nu_{k}+1}, \tilde{\alpha}_{k}(\zeta)\right)$ is analytic in a neighborhood of the origin as a function of $\zeta$, it can be seen, by Theorem S6.1 of Gohberg et al. [4, that such an analytic vector function exists. In terms of $\tilde{\boldsymbol{v}}_{k}(\zeta), V_{1}(z)$ is represented as

$$
V_{1}(z)=\left(\begin{array}{llll}
\tilde{\boldsymbol{v}}_{1}\left(\left(z_{1}-z\right)^{\frac{1}{\nu_{1}+1}}\right) & \tilde{\boldsymbol{v}}_{2}\left(\left(z_{1}-z\right)^{\frac{1}{\nu_{2}+1}}\right) \quad \cdots \quad \tilde{\boldsymbol{v}}_{s_{0}}\left(\left(z_{1}-z\right)^{\frac{1}{\nu_{0}+1}}\right)
\end{array}\right) .
$$

Define $V_{1}^{*}(\zeta)$ as

$$
V_{1}^{*}(\zeta)=V_{1}\left(z_{1}-\zeta^{\nu}\right)=\left(\begin{array}{llll}
\tilde{\boldsymbol{v}}_{1}\left(\zeta^{\frac{\nu}{\nu_{1}+1}}\right) & \tilde{\boldsymbol{v}}_{2}\left(\zeta^{\frac{\nu}{\nu_{2}+1}}\right) & \cdots & \tilde{\boldsymbol{v}}_{s_{0}}\left(\zeta^{\frac{\nu}{\nu_{0}+1}}\right)
\end{array}\right)
$$


which is entry-wise analytic in a neighborhood $\mathbb{U}_{0}$ of the origin, and a point set $\mathcal{E}_{3}^{*}\left(\mathbb{U}_{0}\right)$ as

$$
\mathcal{E}_{3}^{*}\left(\mathbb{U}_{0}\right)=\left\{\zeta \in \mathbb{U}_{0}: z_{1}-\zeta^{\nu} \in \mathcal{E}_{3}\left(\mathbb{U}_{z_{1}}\right)\right\}
$$

Suppose $z_{1}$ is an accumulation point of $\mathcal{E}_{3}\left(\mathbb{U}_{z_{1}}\right)$. By the definition of $\mathcal{E}_{3}^{*}\left(\mathbb{U}_{0}\right)$, this implies that the origin (point 0) is an accumulation point of $\mathcal{E}_{3}^{*}\left(\mathbb{U}_{0}\right)$. Note that $\operatorname{det} V_{1}^{*}(\zeta)=0$ for every $\zeta \in \mathcal{E}_{3}^{*}\left(\mathbb{U}_{0}\right)$. Since $\operatorname{det} V_{1}^{*}(\zeta)$ is analytic in $\mathbb{U}_{0}$ and $\operatorname{det} V_{1}^{*}(\zeta) \not \equiv 0$, there are no accumulation points of $\mathcal{E}_{3}^{*}\left(\mathbb{U}_{0}\right)$ in $\mathbb{U}_{0}$. This is a contradiction. Therefore, $z_{1}$ is not an accumulation point of $\mathcal{E}_{3}\left(\mathbb{U}_{z_{1}}\right)$. By this result, we can set the neighborhood $\mathbb{U}_{z_{1}}$ so small that $\operatorname{det} V_{1}(z) \neq 0$ for every $z \in \mathbb{U}_{z_{1}} \backslash\left\{z_{1}\right\}$. Hereafter, we assume $\mathbb{U}_{z_{1}}$ satisfies this property.

Let $z_{0}$ be a point in $\mathbb{U}_{z_{1}} \cap \mathbb{C}\left(\underline{z}_{1}^{*}, \bar{z}_{1}^{*}\right.$ ] satisfying the condition of Assumption 4.1 and $z_{t}$ an arbitrary point in $\mathbb{U}_{z_{1}} \backslash\left\{z_{1}\right\}$. Consider an arbitrary path $\xi_{z_{0}, z_{t}}$ on $\mathbb{U}_{z_{1}} \backslash\left\{z_{1}\right\}$ and analytically continue $\check{\alpha}_{k}\left(z_{0}\right)$ $\left(=\alpha_{k}\left(z_{0}\right)\right), k=1,2, \ldots, s_{0}$, along the path $\xi_{z_{0}, z_{t}}$. Then, because of the fact explained at the beginning of the proof, the same result of Lemma 4.4 holds, i.e., for any $z \in \mathbb{U}_{z_{1}} \backslash\left\{z_{1}\right\}$, the set $\left\{\check{\alpha}_{1}(z), \check{\alpha}_{2}(z), \ldots, \check{\alpha}_{s_{0}}(z)\right\}$ is unique and it is identical to the set $\left\{\alpha_{1}(z), \alpha_{2}(z), \ldots, \alpha_{s_{0}}(z)\right\}$. Consider $\check{G}_{1}(z)$ define by formula (4.21). Since $\operatorname{det} V_{1}(z) \neq 0$ for every $z \in \mathbb{U}_{z_{1}} \backslash\left\{z_{1}\right\}$, we have $\operatorname{det} \check{V}_{1}(z) \neq 0$ for every $z \in \xi_{z_{0}, z_{t}}$ and $\check{G}_{1}(z)$ is entry-wise analytic on $\xi_{z_{0}, z_{t}}$. For any $z \in \mathbb{U}_{z_{1}} \backslash\left\{z_{1}\right\}$, since the set $\left\{\check{\alpha}_{1}(z), \check{\alpha}_{2}(z), \ldots, \check{\alpha}_{s_{0}}(z)\right\}$ is unique, this $\check{G}_{1}(z)$ is also unique, i.e., it does not depend on the path along which $\check{G}_{1}(z)$ is continued. Hence, every entry of $\check{G}_{1}(z)$ is a single valued function in $\mathbb{U}_{z_{1}} \backslash\left\{z_{1}\right\}$ and the point $z_{1}$ is not a branch point. Consider a matrix function $G_{1}^{*}(\zeta)$ defined as

$$
G_{1}^{*}(\zeta)=\check{G}_{1}\left(z_{1}-\zeta^{\nu}\right)
$$

By the discussion about $V_{1}^{*}(\zeta)$, we see that $G_{1}^{*}(\zeta)$ is entry-wise analytic in a neighborhood of the origin and entry-wise finite on the boundary of the neighborhood. Hence, $G_{1}^{*}(\zeta)$ is bounded around the origin and this implies that $\check{G}_{1}(z)$ is bounded around the point $z_{1}$. As a result, we see that the point $z_{1}$ is a removable singularity for any entry of $\check{G}_{1}(z)$.

Next, consider the case where $z_{1}=\bar{z}_{1}^{*}$. Let $\mathbb{U}_{\bar{z}_{1}^{*}}$ be a neighborhood of $\bar{z}_{1}^{*}$ satisfying $\left(\mathbb{U}_{\bar{z}_{1}^{*}} \backslash\left\{\bar{z}_{1}^{*}\right\}\right) \cap$ $\mathcal{E}_{1}=\emptyset$. We set $\mathbb{U}_{\bar{z}_{1}^{*}}$ being so small that there exists a positive number $\varepsilon$ such that for every $z \in \mathbb{U}_{\bar{z}_{1}^{*}}$, $\left|\alpha_{k}(z)\right|<\underline{\zeta}_{2}\left(\bar{z}_{1}^{*}\right)-\varepsilon$ for $k \in\left\{1,2, \ldots, s_{0}-1\right\}$ and $\left|\alpha_{k}(z)\right| \geq \underline{\zeta}_{2}\left(\bar{z}_{1}^{*}\right)-\varepsilon$ for $k \in\left\{s_{0}+2, s_{0}+3, \ldots, m\right\}$. It is possible by Lemma 4.3. The point $\bar{z}_{1}^{*}$ is a branch point of $\alpha_{s_{0}}(z)$ with order one and $\alpha_{s_{0}}(z)$ coalesces with $\alpha_{s_{0}+1}(z)$ at $z=\bar{z}_{1}^{*}$. Therefore, we focus on two sets of solutions to $\phi(z, w)=0$ : $\left\{\alpha_{1}(z), \ldots, \alpha_{s_{0}-1}(z), \alpha_{s_{0}}(z)\right\}$ and $\left\{\alpha_{1}(z), \ldots, \alpha_{s_{0}-1}(z), \alpha_{s_{0}+1}(z)\right\}$. Further, let the neighborhood $\mathbb{U}_{\bar{z}_{1}^{*}}$ be so small that $\operatorname{det} V_{1}(z) \neq 0$ for every $z \in \mathbb{U}_{\bar{z}_{1}^{*}} \backslash\left\{\bar{z}_{1}^{*}\right\}$. It is possible because of the same reason as that used in the case of $z_{1} \neq \bar{z}_{1}^{*}$. Note that, in this case, $V_{1}(z)$ may be generated from the set $\left\{\alpha_{1}(z), \ldots, \alpha_{s_{0}-1}(z), \alpha_{s_{0}+1}(z)\right\}$. Let $z_{0}$ be a point in $\mathbb{U}_{\bar{z}_{1}^{*}} \cap \mathbb{C}\left(\underline{z}_{1}^{*}, \bar{z}_{1}^{*}\right]$ satisfying the condition of Assumption 4.1 and $z_{t}$ an arbitrary point in $\mathbb{U}_{\bar{z}_{1}^{*}} \backslash\left\{\bar{z}_{1}^{*}\right\}$. Consider an arbitrary path $\xi_{z_{0}, z_{t}}$ on $\mathbb{U}_{\bar{z}_{1}^{*}} \backslash\left\{\bar{z}_{1}^{*}\right\}$ and analytically continue $\check{\alpha}_{k}\left(z_{0}\right)\left(=\alpha_{k}\left(z_{0}\right)\right), k=1,2, \ldots, s_{0}$, along the path $\xi_{z_{0}, z_{t}}$. Then, for any $z \in \xi_{z_{0}, z_{t}}$, the set $\left\{\check{\alpha}_{1}(z), \check{\alpha}_{2}(z), \ldots, \check{\alpha}_{s_{0}}(z)\right\}$ is given by $\left\{\alpha_{1}(z), \ldots, \alpha_{s_{0}-1}(z), \alpha_{s_{0}}(z)\right\}$ or $\left\{\alpha_{1}(z), \ldots, \alpha_{s_{0}-1}(z), \alpha_{s_{0}+1}(z)\right\}$. Consider $\breve{G}_{1}(z)$ define by formula (4.21). We have $\operatorname{det} \check{V}_{1}(z) \neq 0$ for every $z \in \xi_{z_{0}, z_{t}}$ and $\breve{G}_{1}(z)$ is entry-wise analytic on $\xi_{z_{0}, z_{t}}$. Because of the same reason as that used in the case of $z_{1} \neq \bar{z}_{1}^{*}, \check{G}_{1}(z)$ is bounded around $\bar{z}_{1}^{*}$. For any $z \in \mathbb{U}_{\bar{z}_{1}^{*}} \backslash\left\{\bar{z}_{1}^{*}\right\}$, if $\left\{\check{\alpha}_{1}(z), \check{\alpha}_{2}(z), \ldots, \check{\alpha}_{s_{0}}(z)\right\}$ is given by $\left\{\alpha_{1}(z), \ldots, \alpha_{s_{0}-1}(z), \alpha_{s_{0}}(z)\right\}, \check{G}_{1}(z)$ is given by $G_{1}(z)$; if $\left\{\check{\alpha}_{1}(z), \check{\alpha}_{2}(z), \ldots, \check{\alpha}_{s_{0}}(z)\right\}$ is given by $\left\{\alpha_{1}(z), \ldots, \alpha_{s_{0}-1}(z), \alpha_{s_{0}+1}(z)\right\}$, it is given by $G_{1}^{c o}(z)$. Hence, every entry of $\breve{G}_{1}(z)$ is a two valued function in $\mathbb{U}_{\bar{z}_{1}^{*}} \backslash\left\{\bar{z}_{1}^{*}\right\}$ and the point $\bar{z}_{1}^{*}$ is a branch point of $\check{G}_{1}(z)$ with order one. 
Remark 4.4. From the proof of Lemma 4.7, we see that, in a neighborhood of $\bar{z}_{1}^{*}$, the eigenvalue $\alpha_{s_{0}}(z)$ of $G_{1}(z)$ and the corresponding eigenvector $\boldsymbol{v}_{s_{0}}(z)$ are given as

$$
\alpha_{s_{0}}(z)=\tilde{\alpha}_{s_{0}}\left(\left(\bar{z}_{1}^{*}-z\right)^{\frac{1}{2}}\right), \quad \boldsymbol{v}_{s_{0}}(z)=\tilde{\boldsymbol{v}}_{s_{0}}\left(\left(\bar{z}_{1}^{*}-z\right)^{\frac{1}{2}}\right),
$$

where $\tilde{\alpha}_{s_{0}}(\zeta)$ and $\tilde{\boldsymbol{v}}_{s_{0}}(\zeta)$ are analytic in a neighborhood of the origin. We will use this point in the following section.

\section{$5 \quad$ Asymptotics}

\subsection{Way to obtain the asymptotic formulae}

We introduce the following notation. For $r>0, \varepsilon>0$ and $\theta \in[0, \pi / 2)$, define

$$
\tilde{\Delta}_{r}(\varepsilon, \theta)=\{z \in \mathbb{C}:|z|<r+\varepsilon, z \neq r,|\arg (z-r)|>\theta\} .
$$

For $r>0$, we denote by " $\tilde{\Delta}_{r} \ni z \rightarrow r$ " that $\tilde{\Delta}_{r}(\varepsilon, \theta) \ni z \rightarrow r$ for some $\varepsilon>0$ and $\theta \in[0, \pi / 2)$. We use the following lemma for obtaining the directional exact asymptotic formulae of the stationary distribution of the 2D-QBD process, described in Theorem 2.1.

Lemma 5.1 (Theorem VI.4 of Flajolet and Sedgewick [1]). Let $f$ be a generating function of a sequence of real numbers $\left\{a_{n}, n \in \mathbb{Z}_{+}\right\}$, i.e., $f(z)=\sum_{n=0}^{\infty} a_{n} z^{n}$. If $f(z)$ is singular at $z=z_{0}>0$ and analytic on the set $\tilde{\Delta}_{z_{0}}(\varepsilon, \theta)$ for some $\varepsilon>0$ and $\theta \in[0, \pi / 2)$ and if it satisfies

$$
\lim _{\tilde{\Delta}_{z_{0}} \ni z \rightarrow z_{0}}\left(z_{0}-z\right)^{\alpha} f(z)=c_{0}
$$

for $\alpha \in \mathbb{R} \backslash\{0,-1,-2, \ldots\}$ and some nonzero constant $c_{0} \in \mathbb{R}$, then

$$
\lim _{n \rightarrow \infty}\left(\frac{n^{\alpha-1}}{\Gamma(\alpha)} z_{0}^{-n}\right)^{-1} a_{n}=c
$$

for some real number $c$, where $\Gamma(z)$ is the gamma function. This means that the exact asymptotic formula of the sequence $\left\{a_{n}\right\}$ is given by $n^{\alpha-1} z_{0}^{-n}$.

Remark 5.1. In Lemma 5.1, if the value of $\alpha$ satisfying equation (5.1) is 0, then replace $f(z)$ in equation (5.1) with $f_{1}(z)=\left(f(z)-f\left(z_{0}\right)\right)$ and seek $\alpha$ satisfying equation (5.1). Furthermore, if the obtained value of $\alpha$ is a negative integer, say $\alpha=k \in\{-1,-2, \ldots\}$, then replace $f_{1}(z)$ with $f_{2}(z)=$ $\left(f_{1}(z)-\hat{f}_{1}\left(z_{0}\right)\right)$ and seek $\alpha$ satisfying equation (5.1) again, where $\hat{f}_{1}\left(z_{0}\right)=\lim _{\tilde{\Delta}_{z_{0}} \ni z \rightarrow z_{0}}\left(z_{0}-z\right)^{k} f_{1}(z)$. Repeat this procedure until the value of $\alpha$ not being a negative integer is obtained. Then, the obtained value of $\alpha$ satisfies equation (5.2). For example, if $z_{0}$ is a branch point of $f(z)$ with order one, the Puiseux series expansion for $f(z)$ around $z=z_{0}$ is represented as $f(z)=\sum_{n=0}^{\infty} \tilde{a}_{n}\left(z_{0}-z\right)^{\frac{n}{2}}$ and $\alpha$ is given by $\alpha=-\frac{1}{2} \min \left\{k \in \mathbb{Z}_{+}: \tilde{a}_{k} \neq 0\right.$ and $k$ is an odd number $\}$.

In order to apply Lemma 5.1 to the vector generating function $\varphi_{1}(z)$ (resp. $\varphi_{2}(z)$ ), we analytically extend $\varphi_{1}(z)\left(\operatorname{resp} . \varphi_{2}(z)\right)$ over $\tilde{\Delta}_{r_{1}}(\varepsilon, \theta)\left(\operatorname{resp} . \tilde{\Delta}_{r_{2}}(\varepsilon, \theta)\right)$ for some $\varepsilon>0$ and $\theta \in[0, \pi / 2)$ in Lemma 5.2 of Subsection 5.2. It is also clarified that the point $z=r_{1}$ (resp. $z=r_{2}$ ) is the unique singularity of $\varphi_{1}(z)$ (resp. $\left.\varphi_{2}(z)\right)$ on the circle $\partial \Delta\left(0, r_{1}\right)$ (resp. $\partial \Delta\left(0, r_{1}\right)$ ). For the case of Type I $\left(\psi_{1}\left(\bar{z}_{1}^{*}\right)>1\right)$ and that of Type III, we reveal that the point $z=r_{1}$ is a pole of $\boldsymbol{\varphi}_{1}(z)$ with order one 
and give its Laurent series expansion in Lemma 5.3 of Subsection 5.3. The corresponding result for $\varphi_{2}(z)$ is stated in Corollary 5.1. In Lemma 5.4 of Subsection 5.6, we give the Puiseux series expansions for $\varphi_{1}(z)$ in the case of Type I. In that case, if $\psi_{1}\left(\bar{z}_{1}^{*}\right)=1$, then the point $z=r_{1}$ is a pole of $\varphi_{1}(z)$ with order one and also a branch point with order one; if $\psi_{1}\left(\bar{z}_{1}^{*}\right)<1$, then it is just a branch point of $\varphi_{1}(z)$ with order one. In the case of Type I, if $\psi_{2}\left(\bar{z}_{1}^{*}\right) \leq 1$, then analogous results hold for $\varphi_{2}(z)$. In Lemma 5.5 of Subsection 5.6, we give the Laurent and Puiseux series expansions of $\varphi_{1}(z)$ for the case of Type II. In that case, if $\eta_{2}^{(c)}<\theta_{2}^{(c)}$, then the point $z=r_{1}$ is a pole with order one; if $\eta_{2}^{(c)}=\theta_{2}^{(c)}$ and $\psi_{1}\left(\bar{z}_{1}^{*}\right)>1$, then it is a pole with order two; if $\eta_{2}^{(c)}=\theta_{2}^{(c)}$ and $\psi_{1}\left(\bar{z}_{1}^{*}\right)=1$, then it is a pole with order two and also a branch point with order one; otherwise $\left(\eta_{2}^{(c)}=\theta_{2}^{(c)}\right.$ and $\left.\psi_{1}\left(\bar{z}_{1}^{*}\right)<1\right)$, it is a pole with order one and also a branch point with order one. With respect to $\varphi_{2}(z)$ in the case of Type III, results analogous to those for $\varphi_{1}(z)$ in the case of Type II hold.

As a result, from Lemmas 5.1 through 5.5 and Remark 5.1, the exact asymptotic formulae in Theorem 2.1 (main theorem) are automatically obtained.

\subsection{Analytic extension of $\varphi_{1}(z)$ and $\varphi_{2}(z)$}

Recall that the vector generating function $\varphi_{1}(z)$ is entry-wise analytic in the open disk $\Delta_{r_{1}}=$ $\Delta\left(0, r_{1}\right)$, where $r_{1}$ is the radius of convergence of $\varphi_{1}(z)$. We analytically extend it over $\tilde{\Delta}_{r_{1}}(\varepsilon, \theta)$ for some $\varepsilon>0$ and $\theta \in[0, \pi / 2)$, by using equation (3.5) of Lemma 3.3, $\varphi_{2}(z)$ is also extended by using equation (3.8). The following lemma states those points.

Lemma 5.2. $\varphi_{1}(z)$ (resp. $\left.\varphi_{2}(z)\right)$ can analytically be extended over $\tilde{\Delta}_{r_{1}}(\varepsilon, \theta)$ (resp. $\tilde{\Delta}_{r_{2}}(\varepsilon, \theta)$ ) for some $\varepsilon>0$ and $\theta \in[0, \pi / 2)$. The extended $\varphi_{1}(z)$ (resp. $\left.\varphi_{2}(z)\right)$ is entry-wise analytic on $\partial \Delta_{r_{1}} \backslash\left\{r_{1}\right\}$ (resp. $\left.\partial \Delta_{r_{2}} \backslash\left\{r_{2}\right\}\right)$.

Since discussion for $\varphi_{2}(z)$ proceeds in parallel to that for $\varphi_{1}(z)$, we prove the lemma only for $\varphi_{1}(z)$. Before doing it, we present the following propositions. Their proofs are given in Appendix E.

Proposition 5.1. If $G_{1}(z)$ is entry-wise analytic and satisfies $\operatorname{spr}\left(G_{1}(z)\right)<r_{2}$ in a region $\Omega \subset \mathbb{C}$, then $\varphi_{2}\left(G_{1}(z)\right)$ and $\varphi_{2}^{\hat{C}_{2}}\left(z, G_{1}(z)\right)$ are also entry-wise analytic in the same region $\Omega$.

Proposition 5.2. Under Assumption [2.2, for $z \in \mathbb{C}\left(\underline{z}_{1}^{*}, \bar{z}_{1}^{*}\right]$ such that $z \neq|z|$, we have

$$
\operatorname{spr}\left(C_{1}\left(z, G_{1}(z)\right)\right)<\operatorname{spr}\left(C_{1}\left(|z|, G_{1}(|z|)\right)\right) .
$$

Proof of Lemma 5.2. Since $\varphi_{1}(z)$ is entry-wise analytic in $\Delta_{r_{1}}$, it suffices by the identity theorem to show that the right hand side of equation (3.5) is entry-wise analytic on $\partial \Delta_{r_{1}} \backslash\left\{r_{1}\right\}$. The right hand side of equation (3.5) is composed of four terms: $\boldsymbol{\nu}_{0,0}\left(C_{0}\left(z, G_{1}(z)\right)-I\right), \boldsymbol{\varphi}_{2}\left(G_{1}(z)\right)$,

$\varphi_{2}^{\hat{C}_{2}}\left(z, G_{1}(z)\right)$ and $\left(I-C_{1}\left(z, G_{1}(z)\right)\right)^{-1}$. By Lemmas 4.2 and 4.7, $G_{1}(z)$ is entry-wise analytic in $\mathbb{C}\left(\underline{z}_{1}^{*}, \bar{z}_{1}^{*}\right)$ and on $\partial \Delta_{\bar{z}_{1}^{*}} \backslash\left\{\bar{z}_{1}^{*}\right\}$. Hence, if $r_{1}<\bar{z}_{1}^{*}, \boldsymbol{\nu}_{0,0}\left(C_{0}\left(z, G_{1}(z)\right)-I\right)$ is entry-wise analytic on $\partial \Delta_{r_{1}}$; if $r_{1}=\bar{z}_{1}^{*}$, it is entry-wise analytic on $\partial \Delta \bar{z}_{1}^{*} \backslash\left\{\bar{z}_{1}^{*}\right\}$. Analytic properties of the other terms are given as follows.

In the case of Type $I\left(\psi_{1}\left(\bar{z}_{1}^{*}\right)>1\right)$ and that of Type III. Since $r_{1}=e^{\theta_{1}^{(c)}}<\bar{z}_{1}^{*}, G_{1}(z)$ is entry-wise analytic on $\partial \Delta_{r_{1}}$ and by Proposition 2.5] and Lemma 4.1, for $z \in \partial \Delta_{r_{1}}$,

$$
\operatorname{spr}\left(G_{1}(z)\right) \leq \operatorname{spr}\left(G_{1}(|z|)\right)=\underline{\zeta}_{2}(|z|)<\eta_{2}^{(c)}=r_{2} .
$$


Hence, by Proposition 5.1, both $\varphi_{2}\left(G_{1}(z)\right)$ and $\varphi_{2}^{\hat{C}_{2}}\left(z, G_{1}(z)\right)$ are entry-wise analytic on $\partial \Delta_{r_{1}}$. By Proposition [5.2, for $z \in \partial \Delta_{r_{1}} \backslash\left\{r_{1}\right\}$,

$$
\operatorname{spr}\left(C_{1}\left(z, G_{1}(z)\right)\right) \leq \operatorname{spr}\left(\left|C_{1}\left(z, G_{1}(z)\right)\right|\right)<\operatorname{spr}\left(C_{1}\left(r_{1}, G_{1}\left(r_{1}\right)\right)=1,\right.
$$

and we know that $\operatorname{det}\left(I-C_{1}\left(z, G_{1}(z)\right)\right) \neq 0$ at any point on $\partial \Delta_{r_{1}} \backslash\left\{r_{1}\right\}$. Since we have

$$
\left(I-C_{1}\left(z, G_{1}(z)\right)\right)^{-1}=\frac{\operatorname{adj}\left(I-C_{1}\left(z, G_{1}(z)\right)\right)}{\operatorname{det}\left(I-C_{1}\left(z, G_{1}(z)\right)\right)}
$$

where $C_{1}\left(z, G_{1}(z)\right)$ is entry-wise analytic on $\partial \Delta_{r_{1}}$, this implies that each entry of $\left(I-C_{1}\left(z, G_{1}(z)\right)\right)^{-1}$ is analytic on $\partial \Delta_{r_{1}} \backslash\left\{r_{1}\right\}$.

In the case of Type $I\left(\psi_{1}\left(\bar{z}_{1}^{*}\right) \leq 1\right)$. In this case, we have $r_{1}=\bar{z}_{1}^{*}$ and $G_{1}(z)$ is entry-wise analytic on $\partial \Delta_{\bar{z}_{1}^{*}} \backslash\left\{\bar{z}_{1}^{*}\right\}$. In a manner similar to that used in the case of Type I $\left(\psi_{1}\left(\bar{z}_{1}^{*}\right)>1\right)$, we obtain $\operatorname{spr}\left(G_{1}(z)\right)<r_{2}$ for every $z \in \partial \Delta_{\bar{z}_{1}^{*}}$, and by Proposition [5.1, $\varphi_{2}\left(G_{1}(z)\right)$ and $\varphi_{2}^{\hat{C}_{2}}\left(z, G_{1}(z)\right)$ are entry-wise analytic on $\partial \Delta_{\bar{z}_{1}^{*}} \backslash\left\{\bar{z}_{1}^{*}\right\}$. Furthermore, $\left(I-C_{1}\left(z, G_{1}(z)\right)\right)^{-1}$ is also entry-wise analytic on $\partial \Delta_{\bar{z}_{1}^{*}} \backslash\left\{\bar{z}_{1}^{*}\right\}$.

In the case of Type II $\left(\eta_{2}^{(c)}<\theta_{2}^{(c)}\right)$. We have $r_{1}=e^{\bar{\eta}_{1}^{(c)}}<e^{\theta_{1}^{(c)}} \leq \bar{z}_{1}^{*}$ and $G_{1}(z)$ is entry-wise analytic on $\partial \Delta_{r_{1}}$. By Lemma 4.3, if $z \in \partial \Delta_{r_{1}} \backslash\left\{r_{1}\right\}$, the modulus of every eigenvalue of $G_{1}(z)$ is less than $\underline{\zeta}_{2}(|z|)\left(=\underline{\zeta}_{2}\left(r_{1}\right)\right)$. Hence, we have, for $z \in \partial \Delta_{r_{1}} \backslash\left\{r_{1}\right\}$,

$$
\operatorname{spr}\left(G_{1}(z)\right)<\underline{\zeta}_{2}\left(e^{\bar{\eta}_{1}^{(c)}}\right)=e^{\eta_{2}^{(c)}}=r_{2}
$$

and by Proposition [5.1, $\varphi_{2}\left(G_{1}(z)\right)$ and $\varphi_{2}^{\hat{C}_{2}}\left(z, G_{1}(z)\right)$ are entry-wise analytic on $z \in \partial \Delta_{r_{1}} \backslash\left\{r_{1}\right\}$. We know that $\psi_{1}(z)=\operatorname{spr}\left(C_{1}\left(z, G_{1}(z)\right)\right)$ is convex in $z \in \mathbb{R}_{+} \backslash\{0\}$ and $\psi_{1}(1)=\psi_{1}\left(e^{\theta_{1}^{(c)}}\right)=1$. Therefore, we have, for $z \in \partial \Delta_{r_{1}}$,

$$
\operatorname{spr}\left(C_{1}\left(z, G_{1}(z)\right)\right) \leq \operatorname{spr}\left(C_{1}\left(r_{1}, G_{1}\left(r_{1}\right)\right)\right)<\operatorname{spr}\left(C_{1}\left(e^{\theta_{1}^{(c)}}, G_{1}\left(e^{\theta_{1}^{(c)}}\right)\right)\right)=1,
$$

and $\left(I-C_{1}\left(z, G_{1}(z)\right)\right)^{-1}$ is entry-wise analytic on $\partial \Delta_{r_{1}}$.

In the case of Type $I I\left(\eta_{2}^{(c)}=\theta_{2}^{(c)}, \psi_{1}\left(\bar{z}_{1}^{*}\right)>1\right)$. In a manner similar to that used in the case of Type II $\left(\eta_{2}^{(c)}<\theta_{2}^{(c)}\right)$, we see that $G_{1}(z)$ is entry-wise analytic on $\partial \Delta_{r_{1}}$ and that $\varphi_{2}\left(G_{1}(z)\right)$ and $\varphi_{2}^{\hat{C}_{2}}\left(z, G_{1}(z)\right)$ are entry-wise analytic on $\partial \Delta_{r_{1}} \backslash\left\{r_{1}\right\}$. Furthermore, in a manner similar to that used in the case of Type I $\left(\psi_{1}\left(\bar{z}_{1}^{*}\right)>1\right)$, we see that $\left(I-C_{1}\left(z, G_{1}(z)\right)\right)^{-1}$ is entry-wise analytic on $\partial \Delta_{r_{1}} \backslash\left\{r_{1}\right\}$.

In the case of Type II $\left(\eta_{2}^{(c)}=\theta_{2}^{(c)}, \psi_{1}\left(\bar{z}_{1}^{*}\right) \leq 1\right)$. In this case, we have $r_{1}=e^{\bar{\eta}_{1}^{(c)}}=e^{\theta_{1}^{(c)}}=\bar{z}_{1}^{*}$. Hence, $G_{1}(z)$ is entry-wise analytic on $\partial \Delta_{\bar{z}_{1}^{*}} \backslash\left\{\bar{z}_{1}^{*}\right\}$. In a manner similar to that used in the case of Type II $\left(\eta_{2}^{(c)}<\theta_{2}^{(c)}\right)$, we see that $\varphi_{2}\left(G_{1}(z)\right)$ and $\varphi_{2}^{\hat{C}_{2}}\left(z, G_{1}(z)\right)$ are entry-wise analytic on $\partial \Delta_{\bar{z}_{1}^{*}} \backslash\left\{\bar{z}_{1}^{*}\right\}$. Furthermore, we see that $\left(I-C_{1}\left(z, G_{1}(z)\right)\right)^{-1}$ is entry-wise analytic on $\partial \Delta_{\bar{z}_{1}^{*}} \backslash\left\{\bar{z}_{1}^{*}\right\}$.

As mentioned before, since discussion for $\varphi_{2}(z)$ proceeds in parallel to that for $\varphi_{1}(z)$, we explain only about $\varphi_{1}(z)$ in the following subsection. 


\subsection{Singularity of $\varphi_{1}(z)$ : Type I $\left(\psi_{1}\left(\bar{z}_{1}^{*}\right)>1\right)$ and Type III}

By formula (3.5), $\varphi_{1}(z)$ is represented as

$$
\boldsymbol{\varphi}_{1}(z)=\boldsymbol{g}_{1}(z)\left(I-C_{1}\left(z, G_{1}(z)\right)\right)^{-1}=\frac{\boldsymbol{g}_{1}(z) \operatorname{adj}\left(I-C_{1}\left(z, G_{1}(z)\right)\right)}{f_{1}(1, z)},
$$

where

$$
\begin{aligned}
& \boldsymbol{g}_{1}(z)=\boldsymbol{\varphi}_{2}^{\hat{C}_{2}}\left(z, G_{1}(z)\right)-\boldsymbol{\varphi}_{2}\left(G_{1}(z)\right)+\boldsymbol{\nu}_{0,0} l\left(C_{0}\left(z, G_{1}(z)\right)-I r\right), \\
& f_{1}(\lambda, z)=\operatorname{det}\left(\lambda I-C_{1}\left(z, G_{1}(z)\right)\right) .
\end{aligned}
$$

In the case of Type I $\left(\psi_{1}\left(\bar{z}_{1}^{*}\right)>1\right)$, we have $r_{1}<\bar{z}_{1}^{*}$ and $G_{1}(z)$ is entry-wise analytic at $z=r_{1}$. By Proposition [5.1, since $\operatorname{spr}\left(G_{1}\left(r_{1}\right)\right)=\underline{\zeta}_{2}\left(r_{1}\right)<r_{2}$, both $\varphi_{2}\left(G_{1}(z)\right)$ and $\varphi_{2}^{\hat{C}_{2}}\left(z,, G_{1}(z)\right)$ are also entry-wise analytic at $z=r_{1}$. Therefore, $\boldsymbol{g}_{1}(z), \operatorname{adj}\left(I-C_{1}\left(z, G_{1}(z)\right)\right)$ and $f_{1}(1, z)$ are analytic in a neighborhood of $z=r_{1}$. Furthermore, we know $f_{1}\left(1, r_{1}\right)=0$, and each entry of $\varphi_{1}(z)$ is a meromorphic function in a neighborhood of $z=r_{1}$. For $f_{1}(1, z)$, we give the following proposition.

Proposition 5.3. For $z_{0} \in\left(\underline{z}_{1}^{*}, \bar{z}_{1}^{*}\right)$ such that $\psi_{1}\left(z_{0}\right)=1$, the point $z=z_{0}$ is a zero of $f_{1}(1, z)$ with multiplicity one and we have

$$
\lim _{z \rightarrow z_{0}}\left(z_{0}-z\right)^{-1} f_{1}(1, z)=\psi_{1, z}\left(z_{0}\right) f_{1, \lambda}\left(1, z_{0}\right) \neq 0
$$

where $\psi_{1, z}(z)=(d / d z) \psi_{1}(z)$ and $f_{1, \lambda}(\lambda, z)=(\partial / \partial \lambda) f_{1}(\lambda, z)$.

Proof. $f_{1}(\lambda, z)$ is the characteristic polynomial of $C_{1}\left(z, G_{1}(z)\right)$. Under Assumption 2.2, $C_{1}\left(1, G_{1}(1)\right)$ is irreducible and hence, for $z \in\left[\underline{z}_{1}^{*}, \bar{z}_{1}^{*}\right], C_{1}\left(z, G_{1}(z)\right)$ is also irreducible. This implies that for $z \in\left[\underline{z}_{1}^{*}, \bar{z}_{1}^{*}\right]$, the Perron-Frobenius eigenvalue of $C_{1}\left(z, G_{1}(z)\right)$, given by $\psi_{1}(z)=\operatorname{spr}\left(C_{1}\left(z, G_{1}(z)\right)\right.$, is simple, i.e., the algebraic multiplicity of $\psi_{1}(z)$ is one. Denote by $\lambda^{C_{1}}(z)$ the eigenvalue of $C_{1}\left(z, G_{1}(z)\right)$ corresponding to $\psi_{1}(z)$ when $z \in\left[\underline{z}_{1}^{*}, \bar{z}_{1}^{*}\right]$. Then, $\lambda^{C_{1}}(z)$ is analytic on $\left(\underline{z}_{1}^{*}, \bar{z}_{1}^{*}\right)$, and since $\lambda^{C_{1}}\left(z_{0}\right)=\psi_{1}\left(z_{0}\right)=1$, we have

$$
\lim _{z \rightarrow z_{0}}\left(1-\lambda^{C_{1}}(z)\right)^{-1} f_{1}(1, z)=f_{C_{1}, \lambda}\left(1, z_{0}\right) \neq 0 .
$$

Combining this with the fact that $\lim _{z \rightarrow z_{0}}\left(1-\lambda^{C_{1}}(z)\right) /\left(z_{0}-z\right)=\psi_{1, z}(z)$, we obtain equation (5.6). Since $\psi_{1}(1) \leq 1, \psi_{1}\left(z_{0}\right)=1, \psi_{1}(z) \not \equiv 1$ and $\psi_{1}\left(e^{s}\right)$ is convex in $s \in\left[\log \underline{z}_{1}^{*}, \log \bar{z}_{1}^{*}\right]$, we have $\psi_{1, z}\left(z_{0}\right)>0$, and this completes the proof.

Denote by $\boldsymbol{u}^{C_{1}}(z)$ and $\boldsymbol{v}^{C_{1}}(z)$ the left and right eigenvectors of $C_{1}\left(z, G_{1}(z)\right)$ with respect to the eigenvalue $\lambda^{C_{1}}(z)$, satisfying $\boldsymbol{u}^{C_{1}}(z) \boldsymbol{v}^{C_{1}}(z)=1$. Then, the following proposition holds. Its proof is given in Appendix $\mathrm{F}$.

Proposition 5.4. For $z_{0} \in\left(\underline{z}_{1}^{*}, \bar{z}_{1}^{*}\right)$ such that $\psi_{1}\left(z_{0}\right)=1$,

$$
\operatorname{adj}\left(I-C_{1}\left(z_{0}, G_{1}\left(z_{0}\right)\right)\right)=f_{1, \lambda}\left(1, z_{0}\right) \boldsymbol{v}^{C_{1}}\left(z_{0}\right) \boldsymbol{u}^{C_{1}}\left(z_{0}\right),
$$

where $\boldsymbol{v}^{C_{1}}\left(z_{0}\right)$ and $\boldsymbol{u}^{C_{1}}\left(z_{0}\right)$ are positive.

Since $\varphi_{1}(z)$ is entry-wise meromorphic in a neighborhood of $z=r_{1}$, we obtain the following lemma. 
Lemma 5.3 (Type I $\left(\psi_{1}\left(\bar{z}_{1}^{*}\right)>1\right.$ ) and Type III). In the case of Type I, if $\psi_{1}\left(\bar{z}_{1}^{*}\right)>1$, the pint $z=r_{1}$ is a pole of $\varphi_{1}(z)$ with order one and its Laurent series expansion is represented as

$$
\boldsymbol{\varphi}_{1}(z)=\sum_{k=-1}^{\infty} \varphi_{1, k}^{I}\left(r_{1}-z\right)^{k}
$$

where $\boldsymbol{\varphi}_{1,-1}^{I}=c_{\text {pole }}^{\boldsymbol{\varphi}_{1}} \boldsymbol{u}^{C_{1}}\left(r_{1}\right)>\mathbf{0}^{\top}$ and $c_{\text {pole }}^{\boldsymbol{\varphi}_{1}}=\psi_{1, z}\left(r_{1}\right)^{-1} \boldsymbol{g}_{1}\left(r_{1}\right) \boldsymbol{v}^{C_{1}}\left(r_{1}\right)>0$. In the case of Type III, the same result also holds for $\varphi_{1}(z)$.

Proof. By Proposition [5.3, in both the cases, the point $z=r_{1}$ is probably a pole of $\boldsymbol{\varphi}_{1}(z)$ with order one and its Laurent series expansion is given by formula (5.9). If we have

$$
\varphi_{1,-1}^{I}=\lim _{z \rightarrow r_{1}}\left(r_{1}-z\right) \varphi_{1}(z)=\mathbf{0}^{\top}
$$

then $z=r_{1}$ is a removable singularity and $\varphi_{1}(z)$ is analytic at $z=r_{1}$. This contradicts that $\boldsymbol{\varphi}_{1}(z)$ is singular at $z=r_{1}$. Therefore, $\boldsymbol{\varphi}_{1,-1}^{I} \neq \mathbf{0}^{\top}$ and $z=r_{1}$ is a pole of $\boldsymbol{\varphi}_{1}(z)$ with order one. By Propositions [5.3 and [5.4, we obtain the expression of $\varphi_{1,-1}^{I}$. Note that $\varphi_{1,-1}^{I}$ is nonzero and nonnegative and $\boldsymbol{u}^{C_{1}}\left(r_{1}\right)$ is positive. This implies that $c_{\text {pole }}^{\boldsymbol{\varphi}_{1}}$ is positive.

Since the corresponding result for $\varphi_{2}(z)$ will be used later, we state it as the following corollary.

Corollary 5.1 (Type I $\left(\psi_{2}\left(\bar{z}_{2}^{*}\right)>1\right)$ and Type II). In the case of Type I, if $\psi_{1}\left(\bar{z}_{2}^{*}\right)>1$, then the point $z=r_{2}$ is a pole of $\varphi_{2}(z)$ with order one and its Laurent series expansion is represented as

$$
\boldsymbol{\varphi}_{2}(z)=\sum_{k=-1}^{\infty} \boldsymbol{\varphi}_{2, k}^{I}\left(r_{2}-z\right)^{k}
$$

where $\boldsymbol{\varphi}_{2,-1}^{I}=c_{\text {pole }}^{\boldsymbol{\varphi}_{2}} \boldsymbol{u}^{C_{2}}\left(r_{2}\right)>\mathbf{0}^{\top}$ and $c_{\text {pole }}^{\boldsymbol{\varphi}_{2}}=\psi_{2, z}\left(r_{2}\right)^{-1} \boldsymbol{g}_{2}\left(r_{2}\right) \boldsymbol{v}^{C_{2}}\left(r_{2}\right)>0 ; \boldsymbol{g}_{2}(z)$ is given as $\boldsymbol{g}_{2}(z)=\boldsymbol{\varphi}_{1}^{\hat{C}_{1}}\left(G_{2}(z), z\right)-\boldsymbol{\varphi}_{1}\left(G_{2}(z)\right)+\boldsymbol{\nu}_{0,0}\left(C_{0}\left(G_{2}(z), z\right)-I\right), \boldsymbol{u}^{C_{2}}(z)$ and $\boldsymbol{v}^{C_{2}}(z)$ are the left and right eigenvectors of $C_{2}\left(G_{2}(z), z\right)$ with respect to the eigenvalue $\psi_{2}(z)$, and $\psi_{2, z}(z)=(d / d z) \psi_{2}(z)$. In the case of Type II, the same result also holds for $\varphi_{2}(z)$.

In Subsection 5.6, we consider a series expansion for the vector function $\varphi_{1}(z)$ around the point $z=\bar{z}_{1}^{*}$. To this end, we obtain the Puiseux series expansions for $G_{1}(z), \varphi_{2}\left(G_{1}(z)\right)$ and $\varphi_{2}^{\hat{C}_{2}}\left(z, G_{1}(z)\right)$ around $z=\bar{z}_{1}^{*}$, in the following two subsections.

\subsection{Puiseux series expansion for $G_{1}(z)$}

In Assumption 2.5, we assumed that all the eigenvalues of $G_{1}\left(r_{1}\right)$ are distinct. This assumption seems to be rather strong, but under it, if $r_{1}<\bar{z}_{1}^{*}$, all the eigenvalues of $G_{1}(z)$ are analytic at $z=r_{1}$; if $r_{1}=\bar{z}_{1}^{*}$, those of $G_{1}(z)$ except for one corresponding to $\underline{\zeta}_{2}(z)$ are analytic at $z=\bar{z}_{1}^{*}$. Furthermore, all the eigenvectors of $G_{1}(z)$ are linearly independent in a neighborhood of $z=r_{1}$.

In this subsection, we obtain the Puiseux series expansion for $G_{1}(z)$ around $z=\bar{z}_{1}^{*}$. Let $\alpha_{j}(z), j=1,2, \ldots, s_{0}$, be the eigenvalues of $G_{1}(z)$, counting multiplicity, and without loss of generality, let $\alpha_{s_{0}}(z)$ be the eigenvalue corresponding to $\underline{\zeta}_{2}(z)$ when $z \in\left[\underline{z}_{1}^{*}, \bar{z}_{1}^{*}\right]$. For $j \in\left\{1,2, \ldots, s_{0}\right\}$, let $\boldsymbol{v}_{j}(z)$ be the right eigenvector of $G_{1}(z)$ with respect to the eigenvalue $\alpha_{j}(z)$. Define matrices 
$J_{1}(z)$ and $V_{1}(z)$ as $J_{1}(z)=\operatorname{diag}\left(\alpha_{1}(z), \alpha_{2}(z), \ldots, \alpha_{s_{0}}(z)\right)$ and $V_{1}(z)=\left(\boldsymbol{v}_{1}(z) \quad \boldsymbol{v}_{2}(z) \quad \cdots \quad \boldsymbol{v}_{s_{0}}(z)\right)$, respectively. As mentioned above, under Assumption 2.5. $V_{1}(z)$ is nonsingular in a neighborhood of $z=r_{1}$, and $G_{1}(z)$ is factorized as $G_{1}(z)=V_{1}(z) J_{1}(z) V_{1}(z)^{-1}$ (Jordan decomposition). We represent $V_{1}(z)^{-1}$ as

$$
V_{1}(z)^{-1}=\left(\begin{array}{c}
\boldsymbol{u}_{1}(z) \\
\boldsymbol{u}_{2}(z) \\
\vdots \\
\boldsymbol{u}_{s_{0}}(z)
\end{array}\right),
$$

where each $\boldsymbol{u}_{j}(z)$ is a $1 \times s_{0}$ vector. For $j \in\left\{1,2, \ldots, s_{0}\right\}, \boldsymbol{u}_{j}(z)$ is the left eigenvector of $G_{1}(z)$ with respect to the eigenvalue $\alpha_{j}(z)$ and satisfies $\boldsymbol{u}_{j}(z) \boldsymbol{v}_{k}(z)=\delta_{j k}$ for $k \in\left\{1,2, \ldots, s_{0}\right\}$, where $\delta_{j k}$ is the Kronecker delta. Furthermore, from the results of Section 4, we see that, under Assumption 2.5, if $r_{1}<\bar{z}_{1}^{*}, J_{1}(z)$ and $V_{1}(z)$ are entry-wise analytic in a neighborhood of $z=r_{1}$; if $r_{1}=\bar{z}_{1}^{*}, J_{1}(z)$ and $V_{1}(z)$ except for $\alpha_{s_{0}}(z)$ and $\boldsymbol{v}_{s_{0}}(z)$ are also entry-wise analytic in a neighborhood of $z=\bar{z}_{1}^{*}$, where $\alpha_{s_{0}}(z)$ and $\boldsymbol{v}_{s_{0}}(z)$ are given in terms of a function $\tilde{\alpha}_{s_{0}}(\zeta)$ and vector function $\tilde{\boldsymbol{v}}_{s_{0}}(\zeta)$ being analytic in a neighborhood of the origin as $\alpha_{s_{0}}(z)=\tilde{\alpha}_{s_{0}}\left(\left(\bar{z}_{1}^{*}-z\right)^{\frac{1}{2}}\right)$ and $\boldsymbol{v}_{s_{0}}(z)=\tilde{\boldsymbol{v}}_{s_{0}}\left(\left(\bar{z}_{1}^{*}-z\right)^{\frac{1}{2}}\right)$ (see Remark 4.4). Here, we give another representation of $G_{1}(z)$ around $z=\bar{z}_{1}^{*}$. Define $\tilde{J}_{1}(\zeta)$ and $\tilde{V}_{1}(\zeta)$ as

$$
\begin{aligned}
& \tilde{J}_{1}(\zeta)=\operatorname{diag}\left(\alpha_{1}\left(\bar{z}_{1}^{*}-\zeta^{2}\right), \ldots, \alpha_{s_{0}-1}\left(\bar{z}_{1}^{*}-\zeta^{2}\right), \tilde{\alpha}_{s_{0}}(\zeta)\right), \\
& \tilde{V}_{1}(\zeta)=\left(\begin{array}{llll}
\boldsymbol{v}_{1}\left(\bar{z}_{1}^{*}-\zeta^{2}\right) & \cdots & \boldsymbol{v}_{s_{0}-1}\left(\bar{z}_{1}^{*}-\zeta^{2}\right) & \tilde{\boldsymbol{v}}_{s_{0}}(\zeta)
\end{array}\right) .
\end{aligned}
$$

Under Assumption 2.5, $\tilde{J}_{1}(\zeta)$ and $\tilde{V}_{1}(\zeta)$ are entry-wise analytic in a neighborhood of the origin and $\tilde{V}_{1}(\zeta)$ is nonsingular. Therefore, $\tilde{G}_{1}(\zeta)$ defined as $\tilde{G}_{1}(\zeta)=\tilde{V}_{1}(\zeta) \tilde{J}_{1}(\zeta) \tilde{V}_{1}(\zeta)^{-1}$ is entry-wise analytic in a neighborhood of the origin and satisfies

$$
G_{1}(z)=\tilde{G}_{1}\left(\left(\bar{z}_{1}^{*}-z\right)^{\frac{1}{2}}\right) .
$$

Since $\tilde{\alpha}_{s_{0}}(\zeta)$ and $\tilde{G}_{1}(\zeta)$ are analytic in a neighborhood of the origin, their Taylor expansions are represented as

$$
\tilde{\alpha}_{s_{0}}(\zeta)=\sum_{k=0}^{\infty} \tilde{\alpha}_{s_{0}, k} \zeta^{k}, \quad \tilde{G}_{1}(\zeta)=\sum_{k=0}^{\infty} \tilde{G}_{1, k} \zeta^{k},
$$

where $\tilde{\alpha}_{s_{0}, 0}=\underline{\zeta}_{2}\left(\bar{z}_{1}^{*}\right)$ and $\tilde{G}_{1,0}=G_{1}\left(\bar{z}_{1}^{*}\right)$. The Puiseux series expansion for $G_{1}(z)$ around $z=\bar{z}_{1}^{*}$ is represented as

$$
G_{1}(z)=\sum_{k=0}^{\infty} \tilde{G}_{1, k}\left(\bar{z}_{1}^{*}-z\right)^{\frac{k}{2}}
$$

Considering relation between $\bar{\zeta}_{1}(w)$ and $\underline{\zeta}_{2}(z)$, we obtain the following result in a manner similar to that used in the proof of Lemma 10 of Kobayashi and Miyazawa [8].

Proposition 5.5. We have

$$
\lim _{\tilde{\Delta}_{\bar{z}_{1}^{*}} \ni z \rightarrow \bar{z}_{1}^{*}} \frac{\alpha_{s_{0}}\left(\bar{z}_{1}^{*}\right)-\alpha_{s_{0}}(z)}{\left(\bar{z}_{1}^{*}-z\right)^{\frac{1}{2}}}=-\tilde{\alpha}_{s_{0}, 1}=\frac{\sqrt{2}}{\sqrt{-\bar{\zeta}_{1, w^{2}}\left(\underline{\zeta}_{2}\left(\bar{z}_{1}^{*}\right)\right)}}>0,
$$

where $\bar{\zeta}_{1, w^{2}}(w)=\left(d^{2} / d w^{2}\right) \bar{\zeta}_{1}(w)$. 
For $G_{1}(z)$, we give the following proposition, where $\tilde{\alpha}_{s_{0}, 1}$ and $\boldsymbol{u}_{s_{0}}(z)$ are denoted by $\tilde{\alpha}_{s_{0}, 1}^{G_{1}}$ and $\boldsymbol{u}_{s_{0}}^{G_{1}}(z)$, respectively, in order to explicitly indicate that they are the coefficient and vector with respect to $G_{1}(z)$. The proof of the proposition is given in Appendix G.

\section{Proposition 5.6.}

$$
\lim _{\tilde{\Delta}_{\bar{z}_{1}^{*}} \ni z \rightarrow \bar{z}_{1}^{*}} \frac{G_{1}\left(\bar{z}_{1}^{*}\right)-G_{1}(z)}{\left(\bar{z}_{1}^{*}-z\right)^{\frac{1}{2}}}=-\tilde{G}_{1,1}=-\tilde{\alpha}_{s_{0}, 1}^{G_{1}} N_{1}\left(\bar{z}_{1}^{*}\right) \boldsymbol{v}^{R_{1}}\left(\bar{z}_{1}^{*}\right) \boldsymbol{u}_{s_{0}}^{G_{1}}\left(\bar{z}_{1}^{*}\right) \geq \mathbf{0}^{\top}, \neq \mathbf{0}^{\top}
$$

where $\boldsymbol{v}^{R_{1}}\left(\bar{z}_{1}^{*}\right)$ is the right eigenvector of $R_{1}\left(\bar{z}_{1}^{*}\right)$ with respect to the eigenvalue $\bar{\zeta}_{2}\left(\bar{z}_{1}^{*}\right)^{-1}$, satisfying $\boldsymbol{u}_{s_{0}}^{G_{1}}\left(\bar{z}_{1}^{*}\right) N_{1}\left(\bar{z}_{1}^{*}\right) \boldsymbol{v}^{R_{1}}\left(\bar{z}_{1}^{*}\right)=1$.

\subsection{Puiseux series expansions for $\varphi_{2}\left(G_{1}(z)\right)$ and $\varphi_{2}^{\hat{C}_{2}}\left(z,, G_{1}(z)\right)$}

First, we analytically extend $\varphi_{2}\left(G_{1}(z)\right)$ and $\varphi_{2}^{\hat{C_{2}}}\left(z, G_{1}(z)\right)$ around $z=r_{1}$ when $\operatorname{spr}\left(G_{1}\left(r_{1}\right)\right)=$ $\underline{\zeta}_{2}\left(r_{1}\right)=r_{2}<\bar{z}_{2}^{*}$. Recall that, for $z \in \mathbb{C}\left(\underline{z}_{1}^{*}, \bar{z}_{1}^{*}\right)$ such that $\operatorname{spr}\left(G_{1}(z)\right)<r_{2}, \varphi_{2}\left(G_{1}(z)\right)$ and $\varphi_{2}^{\hat{C}_{2}}\left(z, G_{1}(z)\right)$ are entry-wise analytic (see Proposition [5.1). For any $z$ in such a region, $\varphi_{2}\left(G_{1}(z)\right.$ ) is represented as

$$
\begin{aligned}
\boldsymbol{\varphi}_{2}\left(G_{1}(z)\right)^{\top} & =\sum_{k=1}^{\infty}\left(V_{1}(z)^{\top}\right)^{-1} J_{1}(z)^{k} V_{1}(z)^{\top} \boldsymbol{\nu}_{0, k}^{\top} \\
& =\sum_{k=1}^{\infty}\left(\boldsymbol{\nu}_{0, k} \otimes\left(V_{1}(z)^{\top}\right)^{-1} J_{1}(z)^{k}\right) \operatorname{vec}\left(V_{1}(z)^{\top}\right) \\
& =\left(V_{1}(z)^{\top}\right)^{-1} \sum_{k=1}^{\infty}\left(\boldsymbol{\nu}_{0, k} \otimes J_{1}(z)^{k}\right) \operatorname{vec}\left(V_{1}(z)^{\top}\right)
\end{aligned}
$$

where we use the identity $\operatorname{vec}(A B C)=\left(C^{\top} \otimes A\right) \operatorname{vec}(B)$ for matrices $A, B$ and $C$. This equation leads us to

$$
\boldsymbol{\varphi}_{2}\left(G_{1}(z)\right)=\left(\begin{array}{llll}
\boldsymbol{\varphi}_{2}\left(\alpha_{1}(z)\right) \boldsymbol{v}_{1}(z) & \boldsymbol{\varphi}_{2}\left(\alpha_{2}(z)\right) \boldsymbol{v}_{2}(z) & \cdots & \left.\boldsymbol{\varphi}_{2}\left(\alpha_{s_{0}}(z)\right) \boldsymbol{v}_{s_{0}}(z)\right) V(z)^{-1} .
\end{array}\right.
$$

By expression (5.16), we analytically extend $\varphi_{2}\left(G_{1}(z)\right)$ around $z=r_{1}$. Assume $r_{1}<\bar{z}_{1}^{*}$ and $\operatorname{spr}\left(G_{1}\left(r_{1}\right)\right)=r_{2}<\bar{z}_{2}^{*}$. Then, under Assumption 2.5, for $j \in\left\{1,2, \ldots, s_{0}\right\}, \alpha_{j}(z)$ and $\boldsymbol{v}_{j}(z)$ as well as $V(z)^{-1}$ are analytic at $z=r_{1}$. Furthermore, for $j \in\left\{1,2, \ldots, s_{0}-1\right\},\left|\alpha_{j}\left(r_{1}\right)\right|<r_{2}$ and $\boldsymbol{\varphi}_{2}\left(\alpha_{j}(z)\right)$ is analytic at $z=r_{1}$. By Corollary [5.1, since $\alpha_{s_{0}}\left(r_{1}\right)=r_{2}<\bar{z}_{2}^{*}, \varphi_{2}\left(\alpha_{s_{0}}(z)\right)$ is analytic in a neighborhood of $z=r_{1}$ except for the point $z=r_{1}$, which is a pole of $\varphi_{2}\left(\alpha_{s_{0}}(z)\right)$ with order one. Hence, the Laurent series expansion for $\varphi_{2}\left(G_{1}(z)\right)$ around $z=r_{1}$ is represented as

$$
\boldsymbol{\varphi}_{2}\left(G_{1}(z)\right)=\sum_{k=-1}^{\infty} \boldsymbol{\varphi}_{2, k}^{G_{1}}\left(r_{1}-z\right)^{k}
$$

Next, assume $r_{1}=\bar{z}_{1}^{*}$ and define $\tilde{\varphi}_{2}\left(\tilde{G}_{1}(\zeta)\right)$ as

$$
\begin{aligned}
& \tilde{\varphi}_{2}\left(\tilde{G}_{1}(\zeta)\right)=\left(\boldsymbol{\varphi}_{2}\left(\alpha_{1}\left(\bar{z}_{1}^{*}-\zeta^{2}\right)\right) \boldsymbol{v}_{1}\left(\bar{z}_{1}^{*}-\zeta^{2}\right) \cdots\right. \\
& \left.\boldsymbol{\varphi}_{2}\left(\alpha_{s_{0}-1}\left(\bar{z}_{1}^{*}-\zeta^{2}\right)\right) \boldsymbol{v}_{s_{0}-1}\left(\bar{z}_{1}^{*}-\zeta^{2}\right) \boldsymbol{\varphi}_{2}\left(\tilde{\alpha}_{s_{0}}(\zeta)\right) \tilde{\boldsymbol{v}}_{s_{0}}(\zeta)\right) \tilde{V}(\zeta)^{-1} \text {. }
\end{aligned}
$$


If $\operatorname{spr}\left(\tilde{G}_{1}(0)\right)=\operatorname{spr}\left(G_{1}\left(\bar{z}_{1}^{*}\right)\right)<r_{2}$, then $\tilde{\varphi}_{2}\left(\tilde{G}_{1}(\zeta)\right)$ is entry-wise analytic at $\zeta=0$ and the Puiseux series expansion for $\varphi_{2}\left(G_{1}(z)\right)$ around $z=\bar{z}_{1}^{*}$ is represented as

$$
\varphi_{2}\left(G_{1}(z)\right)=\tilde{\varphi}_{2}\left(\tilde{G}_{1}\left(\left(\bar{z}_{1}^{*}-z\right)^{\frac{1}{2}}\right)\right)=\sum_{k=0}^{\infty} \tilde{\varphi}_{2, k}^{\tilde{G}_{1}}\left(\bar{z}_{1}^{*}-z\right)^{\frac{k}{2}}
$$

where $\tilde{\boldsymbol{\varphi}}_{2,0}^{\tilde{G}_{1}}=\boldsymbol{\varphi}_{2}\left(G_{1}\left(\bar{z}_{1}^{*}\right)\right)$. If $\operatorname{spr}\left(\tilde{G}_{1}(0)\right)=\operatorname{spr}\left(G_{1}\left(\bar{z}_{1}^{*}\right)\right)=r_{2}$, then $\zeta=0$ is a pole of $\tilde{\varphi}_{2}\left(\tilde{G}_{1}(\zeta)\right)$ with order one and the Puiseux series expansion for $\varphi_{2}\left(G_{1}(z)\right)$ around $z=\bar{z}_{1}^{*}$ is represented as

$$
\boldsymbol{\varphi}_{2}\left(G_{1}(z)\right)=\tilde{\varphi}_{2}\left(\tilde{G}_{1}\left(\left(\bar{z}_{1}^{*}-z\right)^{\frac{1}{2}}\right)\right)=\sum_{k=-1}^{\infty} \tilde{\varphi}_{2, k}^{\tilde{G}_{1}}\left(\bar{z}_{1}^{*}-z\right)^{\frac{k}{2}} .
$$

From the formulae above, we obtain the following proposition, where $\boldsymbol{u}_{s_{0}}(z)$ and $\boldsymbol{v}_{s_{0}}(z)$ are denoted by $\boldsymbol{u}_{s_{0}}^{G_{1}}(z)$ and $\boldsymbol{v}_{s_{0}}^{G_{1}}(z)$, respectively (the proof is given in Appendix $\underline{\mathrm{H}}$ ).

Proposition 5.7. In the case of Type I, if $r_{1}=\bar{z}_{1}^{*}$, then $\operatorname{spr}\left(G_{1}\left(\bar{z}_{1}^{*}\right)\right)<r_{2}$ and $\tilde{\varphi}_{2,1}^{\tilde{G}_{1}}$ in expansion (5.19) is given by

$$
\tilde{\varphi}_{2,1}^{\tilde{G}_{1}}=\sum_{k=1}^{\infty} \boldsymbol{\nu}_{0, k} \sum_{l=1}^{k} \underline{\zeta}_{2}\left(\bar{z}_{1}^{*}\right)^{k-l} G_{1}\left(\bar{z}_{1}^{*}\right)^{l-1} \tilde{G}_{1,1} \leq \mathbf{0}^{\top}, \neq \mathbf{0}^{\top} .
$$

In the case of Type II, if $r_{1}<\bar{z}_{1}^{*}$, then $\operatorname{spr}\left(G_{1}\left(r_{1}\right)\right)=r_{2}<\bar{z}_{2}^{*}$ and $\varphi_{2,-1}^{G_{1}}$ in expansion (5.17) is given by

$$
\boldsymbol{\varphi}_{2,-1}^{G_{1}}=\underline{\zeta}_{2, z}\left(r_{1}\right)^{-1} c_{p o l e}^{\boldsymbol{\varphi}_{2}} \boldsymbol{u}^{C_{2}}\left(r_{2}\right) \boldsymbol{v}_{s_{0}}^{G_{1}}\left(r_{1}\right) \boldsymbol{u}_{s_{0}}^{G_{1}}\left(r_{1}\right) \geq \mathbf{0}^{\top}, \neq \mathbf{0}^{\top}
$$

if $r_{1}=\bar{z}_{1}^{*}$, then $\operatorname{spr}\left(G_{1}\left(\bar{z}_{1}^{*}\right)\right)=r_{2}<\bar{z}_{2}^{*}$ and $\tilde{\varphi}_{2,-1}^{\tilde{G}_{1}}$ in expansion (5.20) is given by

$$
\tilde{\boldsymbol{\varphi}}_{2,-1}^{\tilde{G}_{1}}=\left(-\tilde{\alpha}_{s_{0}, 1}^{G_{1}}\right)^{-1} c_{\text {pole }}^{\boldsymbol{\varphi}_{2}} \boldsymbol{u}^{C_{2}}\left(r_{2}\right) \boldsymbol{v}_{s_{0}}^{G_{1}}\left(\bar{z}_{1}^{*}\right) \boldsymbol{u}_{s_{0}}^{G_{1}}\left(\bar{z}_{1}^{*}\right) \geq \mathbf{0}^{\top}, \neq \mathbf{0}^{\top} .
$$

Discussion about $\varphi_{2}^{\hat{C}_{2}}\left(z, G_{1}(z)\right)$ proceeds in parallel to that about $\varphi_{2}\left(G_{1}(z)\right)$. In a manner similar to that used in deriving expression (5.15), we obtain

$$
\boldsymbol{\varphi}_{2}^{\hat{C}_{2}}\left(z, G_{1}(z)\right)^{\top}=\left(V_{1}(z)^{\top}\right)^{-1} \sum_{k=1}^{\infty}\left(\boldsymbol{\nu}_{0, k} \otimes J_{1}(z)^{k}\right) \operatorname{vec}\left(\left(\hat{C}_{2}\left(z, G_{1}(z)\right) V_{1}(z)\right)^{\top}\right),
$$

and this leads us to

$$
\begin{gathered}
\boldsymbol{\varphi}_{2}^{\hat{C}_{2}}\left(z, G_{1}(z)\right)=\left(\boldsymbol{\varphi}_{2}\left(\alpha_{1}(z)\right) C_{2}\left(z, \alpha_{1}(z)\right) \boldsymbol{v}_{1}(z) \boldsymbol{\varphi}_{2}\left(\alpha_{2}(z)\right) C_{2}\left(z, \alpha_{2}(z)\right) \boldsymbol{v}_{2}(z)\right. \\
\left.\cdots \quad \boldsymbol{\varphi}_{2}\left(\alpha_{s_{0}}(z)\right) C_{2}\left(z, \alpha_{s_{0}}(z)\right) \boldsymbol{v}_{s_{0}}(z)\right) V(z)^{-1}
\end{gathered}
$$

Assume $r_{1}<\bar{z}_{1}^{*}$ and $\operatorname{spr}\left(G_{1}\left(r_{1}\right)\right)=r_{2}<\bar{z}_{1}^{*}$. By Corollary [5.1, since $\alpha_{s_{0}}(z)$ is analytic at $z=r_{1}$ and $\alpha_{s_{0}}\left(r_{1}\right)=r_{2}$, the point $z=r_{1}$ is a pole of $\varphi_{2}\left(\alpha_{s_{0}}(z)\right)$ with order one. Hence, the Laurent series expansion for $\varphi_{2}^{\hat{C}_{2}}\left(z, G_{1}(z)\right)$ around $z=r_{1}$ is represented as

$$
\boldsymbol{\varphi}_{2}^{\hat{C}_{2}}\left(z, G_{1}(z)\right)=\sum_{k=-1}^{\infty} \boldsymbol{\varphi}_{2, k}^{\hat{C}_{2}}\left(r_{1}-z\right)^{k}
$$


Assume $r_{1}=\bar{z}_{1}^{*}$ and define $\tilde{\varphi}_{2}^{\hat{C}_{2}}\left(\zeta, \tilde{G}_{1}(\zeta)\right)$ as

$$
\begin{aligned}
& \tilde{\boldsymbol{\varphi}}_{2}^{\hat{C}_{2}}\left(\zeta, \tilde{G}_{1}(\zeta)\right)=\left(\boldsymbol{\varphi}_{2}\left(\alpha_{1}\left(\bar{z}_{1}^{*}-\zeta^{2}\right)\right) C_{2}\left(\bar{z}_{1}^{*}-\zeta^{2}, \alpha_{1}\left(\bar{z}_{1}^{*}-\zeta^{2}\right)\right) \boldsymbol{v}_{1}\left(\bar{z}_{1}^{*}-\zeta^{2}\right) \cdots\right. \\
& \boldsymbol{\varphi}_{2}\left(\alpha_{s_{0}-1}\left(\bar{z}_{1}^{*}-\zeta^{2}\right)\right) C_{2}\left(\bar{z}_{1}^{*}-\zeta^{2}, \alpha_{s_{0}-1}\left(\bar{z}_{1}^{*}-\zeta^{2}\right)\right) \boldsymbol{v}_{s_{0}-1}\left(\bar{z}_{1}^{*}-\zeta^{2}\right) \\
& \left.\boldsymbol{\varphi}_{2}\left(\tilde{\alpha}_{s_{0}}(\zeta)\right) C_{2}\left(\bar{z}_{1}^{*}-\zeta^{2}, \tilde{\alpha}_{s_{0}}(\zeta)\right) \tilde{\boldsymbol{v}}_{s_{0}}(\zeta)\right) \tilde{V}(\zeta)^{-1}
\end{aligned}
$$

If $\operatorname{spr}\left(\tilde{G}_{1}(0)\right)=\operatorname{spr}\left(G_{1}\left(\bar{z}_{1}^{*}\right)\right)<r_{2}$, then $\tilde{\varphi}_{2}^{\hat{C}_{2}}\left(\zeta, \tilde{G}_{1}(\zeta)\right)$ is entry-wise analytic at $\zeta=0$ and the Puiseux series expansion for $\varphi_{2}^{\hat{C}_{2}}\left(z, G_{1}(z)\right)$ around $z=\bar{z}_{1}^{*}$ is represented as

$$
\boldsymbol{\varphi}_{2}^{\hat{C}_{2}}\left(z, G_{1}(z)\right)=\tilde{\varphi}_{2}^{\hat{C}_{2}}\left(\left(\bar{z}_{1}^{*}-z\right)^{\frac{1}{2}}, \tilde{G}_{1}\left(\left(\bar{z}_{1}^{*}-z\right)^{\frac{1}{2}}\right)\right)=\sum_{k=0}^{\infty} \tilde{\varphi}_{2, k}^{\hat{C}_{2}}\left(\bar{z}_{1}^{*}-z\right)^{\frac{k}{2}}
$$

If $\operatorname{spr}\left(\tilde{G}_{1}(0)\right)=r_{2}<\bar{z}_{1}^{*}$, then the point $\zeta=0$ is a pole of $\varphi_{2}^{\hat{C}_{2}}\left(\zeta, \tilde{G}_{1}(\zeta)\right)$ with order one and the Puiseux series expansion for $\varphi_{2}^{\hat{C}_{2}}\left(z, G_{1}(z)\right)$ around $z=\bar{z}_{1}^{*}$ is represented as

$$
\boldsymbol{\varphi}_{2}^{\hat{C}_{2}}\left(z, G_{1}(z)\right)=\tilde{\varphi}_{2}^{\hat{C}_{2}}\left(\left(\bar{z}_{1}^{*}-z\right)^{\frac{1}{2}}, \tilde{G}_{1}\left(\left(\bar{z}_{1}^{*}-z\right)^{\frac{1}{2}}\right)\right)=\sum_{k=-1}^{\infty} \tilde{\varphi}_{2, k}^{\hat{C}_{2}}\left(\bar{z}_{1}^{*}-z\right)^{\frac{k}{2}}
$$

For $\varphi_{2}^{\hat{C}_{2}}\left(z, G_{1}(z)\right)$, we give the following proposition. Since this proposition is proved in a manner similar to that used for proving Proposition [5.7, we omit the proof.

Proposition 5.8. In the case of Type I, if $r_{1}=\bar{z}_{1}^{*}$, then $\operatorname{spr}\left(G_{1}\left(\bar{z}_{1}^{*}\right)\right)<r_{2}$ and $\tilde{\varphi}_{2,1}^{\hat{C}_{2}}$ in expansion (5.28) is given by

$$
\begin{aligned}
\tilde{\boldsymbol{\varphi}}_{2,1}^{\hat{C}_{2}}= & \underline{\zeta}_{2}\left(\bar{z}_{1}^{*}\right)^{-1} \boldsymbol{\varphi}_{2}\left(\underline{\zeta}_{2}\left(\bar{z}_{1}^{*}\right)\right)\left(A_{*, 0}^{(2)}\left(\bar{z}_{1}^{*}\right)+A_{*, 1}^{(2)}\left(\bar{z}_{1}^{*}\right)\left(\underline{\zeta}_{2}\left(\bar{z}_{1}^{*}\right) I+G_{1}\left(\bar{z}_{1}^{*}\right)\right)\right) \tilde{G}_{1,1} \\
& +\sum_{k=1}^{\infty} \boldsymbol{\nu}_{0, k} \hat{C}_{2}\left(\bar{z}_{1}^{*}, G_{1}\left(\bar{z}_{1}^{*}\right)\right) \sum_{l=1}^{k-1} \underline{\zeta}_{2}\left(\bar{z}_{1}^{*}\right)^{k-l-1} G_{1}\left(\bar{z}_{1}^{*}\right)^{l-1} \tilde{G}_{1,1} \leq \mathbf{0}^{\top}, \neq \mathbf{0}^{\top},
\end{aligned}
$$

In the case of Type II, if $r_{1}<\bar{z}_{1}^{*}$, then $\operatorname{spr}\left(G_{1}\left(r_{1}\right)\right)=r_{2}<\bar{z}_{2}^{*}$ and $\varphi_{2,-1}^{\hat{C}_{2}}$ in expansion (5.26) is given by

$$
\boldsymbol{\varphi}_{2,-1}^{\hat{C}_{2}}=\underline{\zeta}_{2, z}\left(r_{1}\right)^{-1} c_{p o l e}^{\boldsymbol{\varphi}_{2}} \boldsymbol{u}^{C_{2}}\left(r_{2}\right) C_{2}\left(r_{1}, r_{2}\right) \boldsymbol{v}_{s_{0}}^{G_{1}}\left(r_{1}\right) \boldsymbol{u}_{s_{0}}^{G_{1}}\left(r_{1}\right) \geq \mathbf{0}^{\top}, \neq \mathbf{0}^{\top}
$$

if $r_{1}=\bar{z}_{1}^{*}$, then $\operatorname{spr}\left(G_{1}\left(\bar{z}_{1}^{*}\right)\right)=r_{2}<\bar{z}_{2}^{*}$ and $\tilde{\varphi}_{2,-1}^{\hat{C}_{2}}$ in expansion (5.29) is given by

$$
\tilde{\boldsymbol{\varphi}}_{2,-1}^{\hat{C}_{2}}=\left(-\tilde{\alpha}_{s_{0}, 1}^{G_{1}}\right)^{-1} c_{\text {pole }}^{\boldsymbol{\varphi}_{2}} \boldsymbol{u}^{C_{2}}\left(r_{2}\right) C_{2}\left(\bar{z}_{1}^{*}, r_{2}\right) \boldsymbol{v}_{s_{0}}^{G_{1}}\left(\bar{z}_{1}^{*}\right) \boldsymbol{u}_{s_{0}}^{G_{1}}\left(\bar{z}_{1}^{*}\right) \geq \mathbf{0}^{\top}, \neq \mathbf{0}^{\top}
$$

\subsection{Singularity of $\varphi_{1}(z)$ in the other cases}

Define $\tilde{\varphi}_{1}(\zeta)$ in a neighborhood of $\zeta=0$ as

$$
\tilde{\boldsymbol{\varphi}}_{1}(\zeta)=\tilde{\boldsymbol{g}}_{1}(\zeta)\left(I-C_{1}\left(\bar{z}_{1}^{*}-\zeta^{2}, \tilde{G}_{1}(\zeta)\right)\right)^{-1}=\frac{\tilde{\boldsymbol{g}}_{1}(\zeta) \operatorname{adj}\left(I-C_{1}\left(\bar{z}_{1}^{*}-\zeta^{2}, \tilde{G}_{1}(\zeta)\right)\right)}{\tilde{f}_{1}(1, \zeta)},
$$


Table 1: Singularity of each terms in formula (3.5) at $z=r_{1}$ (Types I and III)

\begin{tabular}{l|ccc|c} 
& \multicolumn{3}{|c|}{ Type I } & Type III \\
& $\psi_{1}\left(\bar{z}_{1}^{*}\right)>1$ & $\psi_{1}\left(\bar{z}_{1}^{*}\right)=1$ & $\psi_{1}\left(\bar{z}_{1}^{*}\right)<1$ & \\
\hline $\boldsymbol{\nu}_{0,0}\left(C_{0}\left(z, G_{1}(z)\right)-I\right)$ & analytic & branch & branch & analytic \\
$\boldsymbol{\varphi}_{2}\left(G_{1}(z)\right)$ & analytic & branch & branch & analytic \\
$\boldsymbol{\varphi}_{2} \hat{C}_{2}\left(z, G_{1}(z)\right)$ & analytic & branch & branch & analytic \\
$\left(I-C_{1}\left(z, G_{1}(z)\right)\right)^{-1}$ & pole & pole and branch & branch & pole
\end{tabular}

Table 2: Singularity of each terms in formula (3.5) at $z=r_{1}$ (Type II)

\begin{tabular}{l|c|ccc} 
& $\eta_{2}^{(c)}<\theta_{2}^{(c)}$ & \multicolumn{4}{|c}{$\eta_{2}^{(c)}=\theta_{2}^{(c)}$} \\
& & $\psi_{1}\left(\bar{z}_{1}^{*}\right)>1$ & $\psi_{1}\left(\bar{z}_{1}^{*}\right)=1$ & $\psi_{1}\left(\bar{z}_{1}^{*}\right)<1$ \\
\hline $\boldsymbol{\nu}_{0,0}\left(C_{0}\left(z, G_{1}(z)\right)-I\right)$ & analytic & analytic & branch & branch \\
$\boldsymbol{\varphi}_{2}\left(G_{1}(z)\right)$ & pole & pole & pole and branch & pole and branch \\
$\boldsymbol{\varphi}_{2}^{\hat{C}_{2}}\left(z, G_{1}(z)\right)$ & pole & pole & pole and branch & pole and branch \\
$\left(I-C_{1}\left(z, G_{1}(z)\right)\right)^{-1}$ & analytic & pole & pole and branch & branch
\end{tabular}

where

$$
\begin{aligned}
\tilde{\boldsymbol{g}}_{1}(\zeta) & =\tilde{\boldsymbol{\varphi}}_{2}^{\hat{C}_{2}}\left(\zeta, \tilde{G}_{1}(\zeta)\right)-\tilde{\boldsymbol{\varphi}}_{2}\left(\tilde{G}_{1}(\zeta)\right)+\boldsymbol{\nu}_{0,0}\left(C_{0}\left(\bar{z}_{1}^{*}-\zeta^{2}, \tilde{G}_{1}(\zeta)\right)-I\right), \\
\tilde{f}_{1}(\lambda, \zeta) & =\operatorname{det}\left(\lambda I-C_{1}\left(\bar{z}_{1}^{*}-\zeta^{2}, \tilde{G}_{1}(\zeta)\right)\right) .
\end{aligned}
$$

$\tilde{f}_{1}(\lambda, \zeta)$ is the characteristic function of $C_{1}\left(\bar{z}_{1}^{*}-\zeta^{2}, \tilde{G}_{1}(\zeta)\right)$. Let $\tilde{\lambda}^{C_{1}}(\zeta)$ be the eigenvalue of $C_{1}\left(\bar{z}_{1}^{*}-\right.$ $\left.\zeta^{2}, \tilde{G}_{1}(\zeta)\right)$ satisfying $\tilde{\lambda}^{C_{1}}(0)=\psi_{1}\left(\bar{z}_{1}^{*}\right)$. We have $\lambda^{C_{1}}(z)=\tilde{\lambda}^{C_{1}}\left(\left(\bar{z}_{1}^{*}-z\right)^{\frac{1}{2}}\right)$ in a neighborhood of $z=\bar{z}_{1}^{*}$, where $\lambda^{C_{1}}(z)$ is the eigenvalue of $C_{1}\left(z, G_{1}(z)\right)$ corresponding to $\psi_{1}(z)$ when $z \in\left[\underline{z}_{1}^{*}, \bar{z}_{1}^{*}\right]$. For $\tilde{f}_{1}(\zeta)$, we give the following proposition, which corresponds to Proposition 5.3 .

Proposition 5.9. If $\psi_{1}\left(\bar{z}_{1}^{*}\right)=1$, then the point $\zeta=0$ is a zero of $\tilde{f}_{1}(1, \zeta)$ with multiplicity one and we have

$$
\lim _{\zeta \rightarrow 0}-\zeta^{-1} \tilde{f}_{1}(1, \zeta)=\tilde{\lambda}_{\zeta}^{C_{1}}(0) f_{1, \lambda}\left(1, \bar{z}_{1}^{*}\right) \neq 0
$$

where $\tilde{\lambda}_{\zeta}^{C_{1}}(\zeta)=(\partial / \partial \zeta) \tilde{\lambda}^{C_{1}}(\zeta)$ and $\tilde{\lambda}_{\zeta}^{C_{1}}(0)=\boldsymbol{u}^{C_{1}}\left(\bar{z}_{1}^{*}\right) A_{*, 1}^{(1)}\left(\bar{z}_{1}^{*}\right) \tilde{G}_{1,1} \boldsymbol{v}^{C_{1}}\left(\bar{z}_{1}^{*}\right)<0$.

In the proof of Proposition 5.9, we use the following proposition (its proof is given in Appendix I).

Proposition 5.10. For any $z_{0} \in\left[\underline{z}_{1}^{*}, \bar{z}_{1}^{*}\right]$ such that $\psi_{1}\left(z_{0}\right)=1$ and for any $k \in \mathbb{Z}_{+}, \boldsymbol{u}^{C_{1}}\left(z_{0}\right)$ and $\boldsymbol{u}^{C_{1}}\left(z_{0}\right) A_{*, 1}^{(1)}\left(z_{0}\right) N_{1}\left(z_{0}\right) R_{1}\left(z_{0}\right)^{k}$ are positive. Analogously, for any $z_{0} \in\left[\underline{z}_{2}^{*}, \bar{z}_{2}^{*}\right]$ such that $\psi_{2}\left(z_{0}\right)=1$ and for any $k \in \mathbb{Z}_{+}, \boldsymbol{u}^{C_{2}}\left(z_{0}\right)$ and $\boldsymbol{u}^{C_{2}}\left(z_{0}\right) A_{1, *}^{(2)}\left(z_{0}\right) N_{2}\left(z_{0}\right) R_{2}\left(z_{0}\right)^{k}$ are also positive. 
Proof of Proposition 5.9. Under Assumption [2.2, $C_{1}\left(\bar{z}_{1}^{*}, G_{1}\left(\bar{z}_{1}^{*}\right)\right)$ is irreducible and the PerronFrobenius eigenvalue $\psi_{1}\left(\bar{z}_{1}^{*}\right)$ is simple. Hence, the eigenvalue $\tilde{\lambda}^{C_{1}}(0)=\psi_{1}\left(\bar{z}_{1}^{*}\right)$ is also simple and $\tilde{\lambda}^{C_{1}}(\zeta)$ is analytic at $\zeta=0$. Since $\tilde{\lambda}^{C_{1}}(0)=\psi_{1}\left(\bar{z}_{1}^{*}\right)=1$, we have

$$
\lim _{\zeta \rightarrow 0}\left(1-\tilde{\lambda}_{s_{0}}^{C_{1}}(\zeta)\right)^{-1} \tilde{f}_{1}(1, \zeta)=f_{1, \lambda}\left(1, \bar{z}_{1}^{*}\right) \neq 0 .
$$

Combining this with the fact that $\lim _{\zeta \rightarrow 0}\left(1-\tilde{\lambda}^{C_{1}}(\zeta)\right) /(0-\zeta)=\tilde{\lambda}_{\zeta}^{C_{1}}(0)$, we obtain equation (5.34). Let $\tilde{\boldsymbol{u}}^{C_{1}}(\zeta)$ and $\tilde{\boldsymbol{v}}^{C_{1}}(\zeta)$ be the left and right eigenvectors of $C_{1}\left(\bar{z}_{1}^{*}-\zeta^{2}, \tilde{G}_{1}(\zeta)\right)$ with respect to the eigenvalue $\tilde{\lambda}^{C_{1}}(\zeta)$, satisfying $\tilde{\boldsymbol{u}}^{C_{1}}(\zeta) \tilde{\boldsymbol{v}}^{C_{1}}(\zeta)=1$. Since $\tilde{\lambda}^{C_{1}}(0)$ is simple, $\tilde{\boldsymbol{u}}^{C_{1}}(\zeta)$ and $\tilde{\boldsymbol{v}}^{C_{1}}(\zeta)$ are analytic at $\zeta=0$. Through some manipulation, we obtain

$$
\tilde{\lambda}_{\zeta}^{C_{1}}(\zeta)=\tilde{\boldsymbol{u}}^{C_{1}}(\zeta)\left(\frac{d}{d \zeta} C_{1}\left(\bar{z}_{1}^{*}-\zeta^{2}, \tilde{G}_{1}(\zeta)\right)\right) \tilde{\boldsymbol{v}}^{C_{1}}(\zeta)
$$

and this leads us to

$$
\tilde{\lambda}_{\zeta}^{C_{1}}(0)=\tilde{\boldsymbol{u}}^{C_{1}}(0) A_{*, 1}^{(1)}\left(\bar{z}_{1}^{*}\right) \tilde{G}_{1,1} \tilde{\boldsymbol{v}}^{C_{1}}(0)=\boldsymbol{u}^{C_{1}}\left(\bar{z}_{1}^{*}\right) A_{*, 1}^{(1)}\left(\bar{z}_{1}^{*}\right) \tilde{G}_{1,1} \boldsymbol{v}^{C_{1}}\left(\bar{z}_{1}^{*}\right) .
$$

Recall that $\tilde{G}_{1,1}=\tilde{\alpha}_{s_{0}, 1}^{G_{1}} N_{1}\left(\bar{z}_{1}^{*}\right) \boldsymbol{v}^{R_{1}}\left(\bar{z}_{1}^{*}\right) \boldsymbol{u}_{s_{0}}^{G_{1}}\left(\bar{z}_{1}^{*}\right)$, where $\tilde{\alpha}_{s_{0}, 1}^{G_{1}}<0$ and $\boldsymbol{v}^{R_{1}}\left(\bar{z}_{1}^{*}\right)$ and $\boldsymbol{u}_{s_{0}}^{G_{1}}\left(\bar{z}_{1}^{*}\right)$ are nonzero and nonnegative. Since $C_{1}\left(\bar{z}_{1}^{*}, G_{1}\left(\bar{z}_{1}^{*}\right)\right)$ is irreducible, $\boldsymbol{v}^{C_{1}}\left(\bar{z}_{1}^{*}\right)$ is positive and $\boldsymbol{u}_{s_{0}}^{G_{1}}\left(\bar{z}_{1}^{*}\right) \boldsymbol{v}^{C_{1}}\left(\bar{z}_{1}^{*}\right)>$ 0. By Proposition 5.10, $\boldsymbol{u}^{C_{1}}\left(\bar{z}_{1}^{*}\right) A_{*, 1}^{(1)}\left(\bar{z}_{1}^{*}\right) N_{1}\left(\bar{z}_{1}^{*}\right) \boldsymbol{v}^{R_{1}}\left(\bar{z}_{1}^{*}\right)>0$. Hence, we have $\tilde{\lambda}_{\zeta}^{C_{1}}(0)<0$.

The following proposition corresponds to Proposition 5.4. Since it can analogously be proved, we omit its proof.

Proposition 5.11. If $\psi_{1}\left(\bar{z}_{1}^{*}\right)=1$, then

$$
\operatorname{adj}\left(I-C_{1}\left(\bar{z}_{1}^{*}, G_{1}\left(\bar{z}_{1}^{*}\right)\right)\right)=f_{1, \lambda}\left(1, \bar{z}_{1}^{*}\right) \boldsymbol{v}^{C_{1}}\left(\bar{z}_{1}^{*}\right) \boldsymbol{u}^{C_{1}}\left(\bar{z}_{1}^{*}\right),
$$

where $\boldsymbol{v}^{C_{1}}\left(\bar{z}_{1}^{*}\right)$ and $\boldsymbol{u}^{C_{1}}\left(\bar{z}_{1}^{*}\right)$ are positive.

Using the results in the previous subsections, we obtain from expressions (5.5) and (5.33) the following two lemmas (see Tables 1 and 2).

Lemma 5.4 (Type I). (1) Type $I\left(\psi_{1}\left(\bar{z}_{1}^{*}\right)=1\right)$. The point $z=\bar{z}_{1}^{*}$ is a branch point of $\boldsymbol{\varphi}_{1}(z)$ with order one and it is also a pole with order one. The Puiseux series expansion for $\varphi_{1}(z)$ around $z=\bar{z}_{1}^{*}$ is represented as

$$
\left.\boldsymbol{\varphi}_{1}(z)=\tilde{\varphi}_{1}\left(\left(\bar{z}_{1}^{*}-z\right)^{\frac{1}{2}}\right)\right)=\sum_{k=-1}^{\infty} \tilde{\varphi}_{1, k}^{I}\left(\bar{z}_{1}^{*}-z\right)^{\frac{k}{2}},
$$

where

$$
\tilde{\boldsymbol{\varphi}}_{1,-1}^{I}=\left(-\tilde{\lambda}_{\zeta}^{C_{1}}(0)\right)^{-1} \boldsymbol{g}_{1}\left(\bar{z}_{1}^{*}\right) \boldsymbol{v}^{C_{1}}\left(\bar{z}_{1}^{*}\right) \boldsymbol{u}^{C_{1}}\left(\bar{z}_{1}^{*}\right)>\mathbf{0}^{\top} .
$$

(2) Type $I\left(\psi_{1}\left(\bar{z}_{1}^{*}\right)<1\right)$. The point $z=\bar{z}_{1}^{*}$ is a branch point of $\varphi_{1}(z)$ with order one and its Puiseux series expansion is represented as

$$
\left.\boldsymbol{\varphi}_{1}(z)=\tilde{\boldsymbol{\varphi}}_{1}\left(\left(\bar{z}_{1}^{*}-z\right)^{\frac{1}{2}}\right)\right)=\sum_{k=0}^{\infty} \tilde{\varphi}_{1, k}^{I}\left(\bar{z}_{1}^{*}-z\right)^{\frac{k}{2}},
$$


where $\tilde{\varphi}_{1,0}^{I}=\varphi_{1}\left(\bar{z}_{1}^{*}\right)$ and

$$
\begin{aligned}
\tilde{\varphi}_{1,1}^{I}= & \left(\underline{\zeta}_{2}\left(\bar{z}_{1}^{*}\right)^{-1} \boldsymbol{\varphi}_{2}\left(\underline{\zeta}_{2}\left(\bar{z}_{1}^{*}\right)\right)\left(A_{*, 0}^{(2)}\left(\bar{z}_{1}^{*}\right)+A_{*, 1}^{(2)}\left(\bar{z}_{1}^{*}\right)\left(\underline{\zeta}_{2}\left(\bar{z}_{1}^{*}\right) I+G_{1}\left(\bar{z}_{1}^{*}\right)\right)\right)\right. \\
& +\sum_{k=1}^{\infty} \boldsymbol{\nu}_{0, k} \hat{C}_{2}\left(\bar{z}_{1}^{*}, G_{1}\left(\bar{z}_{1}^{*}\right)\right) \sum_{l=1}^{k-1} \underline{\zeta}_{2}\left(\bar{z}_{1}^{*}\right)^{k-l-1} G_{1}\left(\bar{z}_{1}^{*}\right)^{l-1} \\
& -\sum_{k=1}^{\infty} \boldsymbol{\nu}_{0, k} \sum_{l=1}^{k} \underline{\zeta}_{2}\left(\bar{z}_{1}^{*}\right)^{k-l} G_{1}\left(\bar{z}_{1}^{*}\right)^{l-1}+\boldsymbol{\nu}_{0,0} A_{*, 1}^{(0)}\left(\bar{z}_{1}^{*}\right) \\
& \left.+\boldsymbol{\varphi}_{1}\left(\bar{z}_{1}^{*}\right) A_{*, 1}^{(1)}\left(\bar{z}_{1}^{*}\right)\right) \tilde{G}_{1,1}\left(I-C_{1}\left(\bar{z}_{1}^{*}, G_{1}\left(\bar{z}_{1}^{*}\right)\right)^{-1} .\right.
\end{aligned}
$$

Proof. (1) In the case of Type I $\left(\psi_{1}\left(\bar{z}_{1}^{*}\right)=1\right)$, since $r_{1}=\bar{z}_{1}^{*}$ and $\operatorname{spr}\left(\tilde{G}_{1}(0)\right)=\operatorname{spr}\left(G_{1}\left(\bar{z}_{1}^{*}\right)\right)<r_{2}$, $\tilde{\boldsymbol{g}}_{1}(\zeta)$ in formula (5.33) is analytic at $\zeta=0 . \tilde{f}_{1}(1, \zeta)$ in formula (5.33) is also analytic at $\zeta=0$. By Proposition [5.9, since $\psi_{1}\left(\bar{z}_{1}^{*}\right)=1$, the point $\zeta=0$ is a zero of $\tilde{f}_{1}(1, \zeta)$ with multiplicity one. Hence, $\zeta=0$ is probably a pole of $\tilde{\varphi}_{1}(\zeta)$ with order one and the Puiseux series expansion for $\varphi_{1}(z)$ is given by formula (5.39). Derivation of the expression for $\tilde{\varphi}_{1,-1}^{I}$ is given in Appendix J Suppose $\boldsymbol{g}_{1}\left(\bar{z}_{1}^{*}\right) \boldsymbol{v}^{C_{1}}\left(\bar{z}_{1}^{*}\right)=0$. Then, since $\boldsymbol{v}^{C_{1}}\left(\bar{z}_{1}^{*}\right)$ is the right eigenvalue of $C_{1}\left(\bar{z}_{1}^{*}, G_{1}\left(\bar{z}_{1}^{*}\right)\right)$ with respect to the eigenvalue $\psi_{1}\left(\bar{z}_{1}^{*}\right)=1$, we have $\boldsymbol{g}_{1}\left(\bar{z}_{1}^{*}\right) C_{1}\left(\bar{z}_{1}^{*}, G_{1}\left(\bar{z}_{1}^{*}\right)\right)^{k} \boldsymbol{v}^{C_{1}}\left(\bar{z}_{1}^{*}\right)=0$ for any $k \geq 1$, and this leads us to

$$
0=\lim _{K \rightarrow \infty} \sum_{k=0}^{K} \boldsymbol{g}_{1}\left(\bar{z}_{1}^{*}\right) C_{1}\left(\bar{z}_{1}^{*}, G_{1}\left(\bar{z}_{1}^{*}\right)\right)^{k} \boldsymbol{v}^{C_{1}}\left(\bar{z}_{1}^{*}\right)=\boldsymbol{\varphi}_{1}\left(\bar{z}_{1}^{*}\right) \boldsymbol{v}^{C_{1}}\left(\bar{z}_{1}^{*}\right) .
$$

Since $\boldsymbol{v}^{C_{1}}\left(\bar{z}_{1}^{*}\right)>\mathbf{0}^{\top}$, this contradicts to positivity of $\boldsymbol{\varphi}_{1}\left(\bar{z}_{1}^{*}\right)$, where we include the case where some entries of $\boldsymbol{\varphi}_{1}\left(\bar{z}_{1}^{*}\right)$ diverge to positive infinity. Hence, we have $\boldsymbol{g}_{1}\left(\bar{z}_{1}^{*}\right) \boldsymbol{v}^{C_{1}}\left(\bar{z}_{1}^{*}\right) \neq 0$, and $\tilde{\boldsymbol{\varphi}}_{1,-1}^{I}$ is positive. This implies that $\zeta=0$ is definitely a pole of $\tilde{\varphi}_{1}(\zeta)$ with order one.

(2) In the case of Type I $\left(\psi_{1}\left(\bar{z}_{1}^{*}\right)<1\right)$, because of the same reason, $\tilde{\boldsymbol{g}}_{1}(\zeta)$ in formula (5.33) is analytic at $\zeta=0$. Since $\psi_{1}\left(\bar{z}_{1}^{*}\right)<1$, we have $\tilde{f}_{1}(1,0) \neq 0$. Hence, $\tilde{\varphi}_{1}(\zeta)$ is analytic at $\zeta=0$ and the Puiseux series expansion for $\varphi_{1}(z)$ is given by formula (5.41). Derivation of the expression for $\tilde{\varphi}_{1,1}^{I}$ is given in Appendix J.

Lemma 5.5 (Type II). (1) Type II $\left(\eta_{2}^{(c)}<\theta_{2}^{(c)}\right)$. The point $z=r_{1}$ is a pole of $\varphi_{1}(z)$ with order one and its Laurent series expansion is represented as

$$
\boldsymbol{\varphi}_{1}(z)=\sum_{k=-1}^{\infty} \boldsymbol{\varphi}_{1, k}^{I I}\left(r_{1}-z\right)^{k}
$$

where

$$
\boldsymbol{\varphi}_{1,-1}^{I I}=\frac{c_{p o l e}^{\boldsymbol{\varphi}_{2}} \boldsymbol{u}^{C_{2}}\left(r_{2}\right) A_{1, *}^{(2)}\left(r_{2}\right) N_{2}\left(r_{2}\right) \boldsymbol{v}^{R_{2}}\left(r_{2}\right)}{\underline{\zeta}_{2, z}\left(r_{1}\right)} \boldsymbol{u}_{s_{0}}^{G_{1}}\left(r_{1}\right)\left(I-C_{1}\left(r_{1}, G_{1}\left(r_{1}\right)\right)\right)^{-1}>\mathbf{0}^{\top}
$$

and $\boldsymbol{v}^{R_{2}}\left(r_{2}\right)$ is the right eigenvector of $R_{2}\left(r_{2}\right)$ with respect to the eigenvalue $r_{1}^{-1}$.

(2) Type II $\left(\eta_{2}^{(c)}=\theta_{2}^{(c)}\right.$ and $\left.\psi_{1}\left(\bar{z}_{1}^{*}\right)>1\right)$. The point $z=\bar{z}_{1}^{*}$ is a pole of $\varphi_{1}(z)$ with order two and its Laurent series expansion is represented as

$$
\boldsymbol{\varphi}_{1}(z)=\sum_{k=-2}^{\infty} \boldsymbol{\varphi}_{1, k}^{I I}\left(r_{1}-z\right)^{k}
$$


where

$$
\boldsymbol{\varphi}_{1,-2}^{I I}=\frac{c_{\text {pole }}^{\boldsymbol{\varphi}_{2}} \boldsymbol{u}^{C_{2}}\left(r_{2}\right) A_{1, *}^{(2)}\left(r_{2}\right) N_{2}\left(r_{2}\right) \boldsymbol{v}^{R_{2}}\left(r_{2}\right) \boldsymbol{u}_{s_{0}}^{G_{1}}\left(r_{1}\right) \boldsymbol{v}^{C_{1}}\left(r_{1}\right)}{\underline{\zeta}_{2, z}\left(r_{1}\right) \psi_{1, z}\left(r_{1}\right)} \boldsymbol{u}^{C_{1}}\left(r_{1}\right)>\mathbf{0}^{\top} .
$$

(3) Type II $\left(\eta_{2}^{(c)}=\theta_{2}^{(c)}\right.$ and $\left.\psi_{1}\left(\bar{z}_{1}^{*}\right)=1\right)$. The point $z=\bar{z}_{1}^{*}$ is a branch point of $\varphi_{1}(z)$ with order one and it is also a pole with order two. The Puiseux series expansion for $\varphi_{1}(z)$ around $z=\bar{z}_{1}^{*}$ is represented as

$$
\left.\boldsymbol{\varphi}_{1}(z)=\tilde{\varphi}_{1}\left(\left(\bar{z}_{1}^{*}-z\right)^{\frac{1}{2}}\right)\right)=\sum_{k=-2}^{\infty} \tilde{\varphi}_{1, k}^{I I}\left(\bar{z}_{1}^{*}-z\right)^{\frac{k}{2}}
$$

where

$$
\tilde{\boldsymbol{\varphi}}_{1,-2}^{I I}=\frac{c_{p o l e}^{\boldsymbol{\varphi}_{2}} \boldsymbol{u}^{C_{2}}\left(r_{2}\right) A_{1, *}^{(2)}\left(r_{2}\right) N_{2}\left(r_{2}\right) \boldsymbol{v}^{R_{2}}\left(r_{2}\right) \boldsymbol{u}_{s_{0}}^{G_{1}}\left(\bar{z}_{1}^{*}\right) \boldsymbol{v}^{C_{1}}\left(\bar{z}_{1}^{*}\right)}{\tilde{\alpha}_{s_{0}, 1}^{G_{1}} \tilde{\lambda}_{\zeta}^{C_{1}}(0)} \boldsymbol{u}^{C_{1}}\left(\bar{z}_{1}^{*}\right)>\mathbf{0}^{\top}
$$

(4) Type II $\left(\eta_{2}^{(c)}=\theta_{2}^{(c)}\right.$ and $\left.\psi_{1}\left(\bar{z}_{1}^{*}\right)<1\right)$. The point $z=\bar{z}_{1}^{*}$ is a branch point of $\varphi_{1}(z)$ with order one and it is also a pole with order one. The Puiseux series expansion for $\varphi_{1}(z) z=\bar{z}_{1}^{*}$ is represented as

$$
\left.\boldsymbol{\varphi}_{1}(z)=\tilde{\varphi}_{1}\left(\left(\bar{z}_{1}^{*}-z\right)^{\frac{1}{2}}\right)\right)=\sum_{k=-1}^{\infty} \tilde{\varphi}_{1, k}^{I I}\left(\bar{z}_{1}^{*}-z\right)^{\frac{k}{2}}
$$

where

$$
\tilde{\boldsymbol{\varphi}}_{1,-1}^{I I}=\frac{c_{\text {pole }}^{\boldsymbol{\varphi}_{2}} \boldsymbol{u}^{C_{2}}\left(r_{2}\right) A_{1, *}^{(2)}\left(r_{2}\right) N_{2}\left(r_{2}\right) \boldsymbol{v}^{R_{2}}\left(r_{2}\right)}{-\tilde{\alpha}_{s_{0}, 1}^{G_{1}}} \boldsymbol{u}_{s_{0}}^{G_{1}}\left(\bar{z}_{1}^{*}\right)\left(I-C_{1}\left(\bar{z}_{1}^{*}, G_{1}\left(\bar{z}_{1}^{*}\right)\right)\right)^{-1}>\mathbf{0}^{\top} .
$$

Proof. (1) In the case of Type II $\left(\eta_{2}^{(c)}<\theta_{2}^{(c)}\right)$, since $r_{1}=e^{\bar{\eta}_{1}^{(c)}}<e^{\theta_{1}^{(c)}}$, we have $f_{1}\left(1, r_{1}\right) \neq 0$. Since $\operatorname{spr}\left(G_{1}\left(r_{1}\right)\right)=\underline{\zeta}_{2}\left(r_{1}\right)=e^{\eta_{2}^{(c)}}=r_{2}$, the point $z=r_{1}$ is probably a pole of $\boldsymbol{g}_{1}(z)$ with order one. Hence, $z=r_{1}$ is probably a pole of $\varphi_{1}(z)$ with order one and the Laurent series expansion for $\varphi_{1}(z)$ is given by formula (5.43). Derivation of the expression for $\varphi_{1,-1}^{I I}$ is given in Appendix J, By Proposition 5.10, $\boldsymbol{u}^{C_{2}}\left(r_{2}\right) A_{1, *}^{(2)}\left(r_{2}\right) N_{2}\left(r_{2}\right) \boldsymbol{v}^{R_{2}}\left(r_{2}\right)>0$, and we know that $\left(I-C_{1}\left(r_{1}, G_{1}\left(r_{1}\right)\right)\right)^{-1}$ is positive. Hence, $\varphi_{1,-1}^{I I}$ is positive, and this implies that $z=r_{1}$ is definitely a pole of $\varphi_{1}(z)$ with order one.

(2) In the case of Type II $\left(\eta_{2}^{(c)}=\theta_{2}^{(c)}\right.$ and $\left.\psi_{1}\left(\bar{z}_{1}^{*}\right)>1\right)$, since $r_{1}=e^{\bar{\eta}_{1}^{(c)}}=e^{\theta_{1}^{(c)}}<\bar{z}_{1}^{*}$, we have $\psi_{1}\left(r_{1}\right)=1$ and, by Proposition [5.3, the point $z=r_{1}$ is a zero of $f_{1}(1, z)$ with multiplicity one. Because of the same reason as that used in (1), $z=r_{1}$ is probably a pole of $\boldsymbol{g}_{1}(z)$ with order one. Hence, $z=r_{1}$ is probably a pole of $\varphi_{1}(z)$ with order two and the Laurent series expansion for $\boldsymbol{\varphi}_{1}(z)$ is given by formula (5.45). Derivation of the expression for $\boldsymbol{\varphi}_{1,-2}^{I I}$ is given in Appendix J . We have $\boldsymbol{u}^{C_{2}}\left(r_{2}\right) A_{1, *}^{(2)}\left(r_{2}\right) N_{2}\left(r_{2}\right) \boldsymbol{v}^{R_{2}}\left(r_{2}\right)>0$ and $\boldsymbol{u}_{s_{0}}^{G_{1}}\left(r_{1}\right) \boldsymbol{v}^{C_{1}}\left(r_{1}\right)>0$. Hence, $\boldsymbol{\varphi}_{1,-1}^{I I}$ is positive, and this implies that $z=r_{1}$ is definitely a pole of $\varphi_{1}(z)$ with order two.

(3) In the case of Type II $\left(\eta_{2}^{(c)}=\theta_{2}^{(c)}\right.$ and $\left.\psi_{1}\left(\bar{z}_{1}^{*}\right)=1\right)$, we have $r_{1}=e^{\bar{\eta}_{1}^{(c)}}=e^{\theta_{1}^{(c)}}=\bar{z}_{1}^{*}$. Since $\operatorname{spr}\left(\tilde{G}_{1}(0)\right)=\operatorname{spr}\left(G_{1}\left(\bar{z}_{1}^{*}\right)\right)=r_{2}$, the point $\zeta=0$ is probably a pole of $\tilde{\boldsymbol{g}}_{1}(\zeta)$ with order one. By 
Proposition [5.9, since $\psi_{1}\left(\bar{z}_{1}^{*}\right)=1, \zeta=0$ is a zero of $\tilde{f}_{1}(1, \zeta)$ with multiplicity one. Hence, $\zeta=0$ is probably a pole of $\tilde{\varphi}_{1}(\zeta)$ with at most order two and the Puiseux series expansion for $\varphi_{1}(z)$ is given by formula (5.47). Derivation of the expression for $\tilde{\varphi}_{1,-2}^{I I}$ is given in Appendix』. In the same reason as that used in $(2), \tilde{\varphi}_{1,-2}^{I I}$ is positive, and this implies that $\zeta=0$ is definitely a pole of $\tilde{\varphi}_{1}(\zeta)$ with order two.

(4) In the case of Type II $\left(\eta_{2}^{(c)}=\theta_{2}^{(c)}\right.$ and $\left.\psi_{1}\left(\bar{z}_{1}^{*}\right)<1\right)$, we have $r_{1}=\bar{z}_{1}^{*}$ and the point $\zeta=0$ is probably a pole of $\tilde{\boldsymbol{g}}_{1}(\zeta)$ with order one. Since $\psi_{1}\left(\bar{z}_{1}^{*}\right)<1$, we have $\tilde{f}_{1}(1,0) \neq 0$. Hence, $\zeta=0$ is probably a pole of $\tilde{\varphi}_{1}(\zeta)$ with order one and the Puiseux series expansion for $\varphi_{1}(z)$ is given by formula (5.49). Derivation of the expression for $\tilde{\varphi}_{1,-1}^{I I}$ is given in Appendix J] In the same reason as that used in (1), $\tilde{\varphi}_{1,-1}^{I I}$ is positive, and this implies that $\zeta=0$ is definitely a pole of $\tilde{\varphi}_{1}(\zeta)$ with order one.

Remark 5.2. We have not yet clarified whether the coefficient vector $\tilde{\varphi}_{1,1}^{I}$ in Lemma 5.4 is nonzero or not. We leave this point as a further study.

\section{References}

[1] Flajolet, P. and Sedgewick, R., Analytic Combinatorics, Cambridge University Press (2009).

[2] Fayolle, G., Malyshev, V.A., and Menshikov, M.V., Topics in the Constructive Theory of Countable Markov Chains, Cambridge University Press, Cambridge (1995).

[3] Gail, H.R., Hantler, S.L. and Taylor, B.A., Spectral Analysis of M/G/1 and G/M/1 type Markov chain, Advances in Applied Probability 28 (1996), 114-165.

[4] Gohberg, I., Lancaster, P. and Rodman, L., Matrix Polynomials, SIAM, Philadelphia (2009).

[5] Horn, R.A. and Johnson, C.R., Matrix Analysis, Cambridge University Press, Cambridge (1985).

[6] Horn, R.A. and Johnson, C.R., Topics in Matrix Analysis, Cambridge University Press, Cambridge (1991).

[7] Kingman, J.F.C., A convexity property of positive matrixes, Quart. J. Math. Oxford (2), 12 (1961), 283-284.

[8] Kobayashi, M. and Miyazawa, M., Revisit to the tail asymptotics of the double QBD process: Refinement and complete solutions for the coordinate and diagonal directions, Matrix-Analytic Methods in Stochastic Models (2013), 145-185.

[9] Kobayashi, M., Miyazawa, M. and Ozawa, T., Convergence domain of the generating function of the stationary distribution for a discrete-time two-dimensional QBD process, in preparation (2017).

[10] Latouche, G. and Ramaswami, V., Introduction to Matrix Analytic Methods in Stochastic Modeling, SIAM, Philadelphia, 1999.

[11] Li, Q.-L. and Zhao, Y.Q., $\beta$-invariant measures for transition matrices of GI/M/1 type, Stochastic Models 19(2) (2003), 201-233. 
[12] Markushevich, A.I., Theory of Functions of a Complex Variable, AMS Chelsea Publishing (2005).

[13] Miyazawa, M. and Zhao, Y.Q., The stationary tail asymptotics in the GI/G/1 type queue with countably many background states, Advances in Applied Probability 36(4) (2004), 1231-1251.

[14] Miyazawa, M., Tail decay rates in double QBD processes and related reflected random walks, Mathematics of Operations Research 34(3) (2009), 547-575.

[15] M. Miyazawa, Light tail asymptotics in multidimensional reflecting processes for queueing networks, TOP 19(2) (2011), 233-299.

[16] Miyazawa, M., Superharmonic vector for a nonnegative matrix with QBD block structure and its application to a Markov modulated two dimensional reflecting process, Queueing Systems 81 (2015), 1-48.

[17] Neuts, M.F., Matrix-Geometric Solutions in Stochastic Models, Dover Publications, New York (1994).

[18] Ozawa, T., Asymptotics for the stationary distribution in a discrete-time two-dimensional quasi-birth-and-death process, Queueing Systems 74 (2013), 109-149.

[19] Ozawa, T., Stability of multidimensional skip-free Markov modulated reflecting random walks: Revisit to Malyshev and Menshikov's results and application to queueing networks, submitted (2015) (arXive:1208.3043).

[20] Seneta, E., Non-negative Matrices and Markov Chains, revised printing, Springer-Verlag, New York (2006).

[21] R. L. Tweedie, Operator-geometric stationary distributions for Markov chains with applications to queueing models, Advances in Applied Probability 14 (1982), 368-391.

\section{A Proof of Lemma 2.2}

Proof of Lemma 2.2. First we note that, for $n \geq 1, j \in S_{0}$ and $\left(s_{1}, s_{2}\right) \in \mathbb{R}^{2}, C\left(e^{s_{1}}, e^{s_{2}}\right)^{n}$ satisfies

$$
\left[C\left(e^{s_{1}}, e^{s_{2}}\right)^{n}\right]_{j j}=\sum_{\boldsymbol{k}_{(n)} \in \mathbb{H}^{n}} \sum_{\boldsymbol{l}_{(n)} \in \mathbb{H}^{n}}\left[A_{k_{1}, l_{1}} A_{k_{2}, l_{2}} \cdots A_{k_{n}, l_{n}}\right]_{j j} e^{s_{1} \sum_{p=1}^{n} k_{p}+s_{2} \sum_{p=1}^{n} l_{p}}
$$

where $\boldsymbol{k}_{(n)}=\left(k_{1}, k_{2}, \ldots, k_{n}\right)$ and $\boldsymbol{l}_{(n)}=\left(l_{1}, l_{2}, \ldots, l_{n}\right)$. Consider the Markov chain $\left\{\tilde{\boldsymbol{Y}}_{n}\right\}=\left\{\left(\tilde{X}_{1, n}, \tilde{X}_{2, n}, \tilde{J}_{n}\right)\right\}$ generated by $\left\{A_{k, l}, k, l \in \mathbb{H}\right\}$ (see Definition 2.1) and assume that $\left\{\tilde{\boldsymbol{Y}}_{n}\right\}$ starts from the state $(0,0, j)$. Since $\left\{A_{k, l}, k, l \in \mathbb{H}\right\}$ is irreducible and aperiodic, there exists $n_{0} \geq 1$ such that, for every $j \in S_{0}, \mathbb{P}\left(\tilde{\boldsymbol{Y}}_{n_{0}}=(1,0, j) \mid \tilde{\boldsymbol{Y}}_{0}=(0,0, j)\right)>0, \mathbb{P}\left(\tilde{\boldsymbol{Y}}_{n_{0}}=(0,1, j) \mid \tilde{\boldsymbol{Y}}_{0}=(0,0, j)\right)>0$, $\mathbb{P}\left(\tilde{\boldsymbol{Y}}_{n_{0}}=(-1,0, j) \mid \tilde{\boldsymbol{Y}}_{0}=(0,0, j)\right)>0$ and $\mathbb{P}\left(\tilde{\boldsymbol{Y}}_{n_{0}}=(0,-1, j) \mid \tilde{\boldsymbol{Y}}_{0}=(0,0, j)\right)>0$. This implies 
that, for some $\boldsymbol{k}_{1,\left(n_{0}\right)}^{(j)}, \boldsymbol{l}_{1,\left(n_{0}\right)}^{(j)}, \boldsymbol{k}_{2,\left(n_{0}\right)}^{(j)}, \boldsymbol{l}_{2,\left(n_{0}\right)}^{(j)}, \boldsymbol{k}_{3,\left(n_{0}\right)}^{(j)}, \boldsymbol{l}_{3,\left(n_{0}\right)}^{(j)}, \boldsymbol{k}_{4,\left(n_{0}\right)}^{(j)}$ and $\boldsymbol{l}_{4,\left(n_{0}\right)}^{(j)}$ in $\mathbb{H}^{n_{0}}$, we have

$$
\begin{aligned}
& b_{1, j}=\left[A_{k_{1,1}^{(j)}, l_{1,1}^{(j)}} A_{k_{1,2}^{(j)}, l_{1,2}^{(j)}} \cdots A_{k_{1, n_{0}}^{(j)}, l_{1, n_{0}}^{(j)}}\right]_{j j}>0, \quad \sum_{p=1}^{n_{0}} k_{1, p}^{(j)}=1, \quad \sum_{p=1}^{n_{0}} l_{1, p}^{(j)}=0 \\
& b_{2, j}=\left[A_{k_{2,1}^{(j)}, l_{2,1}^{(j)}} A_{k_{2,2}^{(j)}, l_{2,2}^{(j)}} \cdots A_{k_{2, n_{0}}^{(j)}, l_{2, n_{0}}^{(j)}}\right]_{j j}>0, \quad \sum_{p=1}^{n_{0}} k_{2, p}^{(j)}=0, \quad \sum_{p=1}^{n_{0}} l_{2, p}^{(j)}=1, \\
& b_{3, j}=\left[A_{k_{3,1}^{(j)}, l_{3,1}^{(j)}} A_{k_{3,2}^{(j)}, l_{3,2}^{(j)}} \cdots A_{k_{3, n_{0}}^{(j)} l_{3, n_{0}}^{(j)}}\right]_{j j}>0, \quad \sum_{p=1}^{n_{0}} k_{3, p}^{(j)}=-1, \quad \sum_{p=1}^{n_{0}} l_{3, p}^{(j)}=0, \\
& b_{4, j}=\left[A_{k_{4,1}^{(j)}, l_{4,1}^{(j)}} A_{k_{4,2}^{(j)}, l_{4,2}^{(j)}} \cdots A_{k_{4, n_{0}}^{(j)}, l_{4, n_{0}}^{(j)}}\right]_{j j}>0, \quad \sum_{p=1}^{n_{0}} k_{4, p}^{(j)}=0, \quad \sum_{p=1}^{n_{0}} l_{4, p}^{(j)}=-1 .
\end{aligned}
$$

Hence, the sum of any row of $C\left(e^{s_{1}}, e^{s_{2}}\right)^{n_{0}}$ is greater than or equal to $b^{*}\left(e^{s_{1}}+e^{s_{2}}+e^{-s_{1}}+e^{-s_{2}}\right)$, where

$$
b^{*}=\min _{j \in S_{0}} \min _{1 \leq i \leq 4} b_{i, j}>0,
$$

and we obtain

$$
\chi\left(e^{s_{1}}, e^{s_{2}}\right)=\operatorname{spr}\left(C\left(e^{s_{1}}, e^{s_{2}}\right)\right)=\operatorname{spr}\left(C\left(e^{s_{1}}, e^{s_{2}}\right)^{n_{0}}\right)^{\frac{1}{n_{0}}} \geq\left(b^{*}\left(e^{s_{1}}+e^{s_{2}}+e^{-s_{1}}+e^{-s_{2}}\right)\right)^{\frac{1}{n_{0}}},
$$

where we use the fact that, for a nonnegative square matrix $A=\left(a_{i, j}\right), \operatorname{spr}(A) \geq \min _{i} \sum_{j} a_{i, j}(\operatorname{see}$, for example, Theorem 8.1.22 of Horn and Johnson [5]). This means that $\chi\left(e^{s_{1}}, e^{s_{2}}\right)$ is unbounded in any direction, and since $\chi\left(e^{s_{1}}, e^{s_{2}}\right)$ is convex in $\left(s_{1}, s_{2}\right)$, we see that $\bar{\Gamma}$ is a bounded set.

\section{B Proof of Lemma 3.2}

Proof. We denote by $J_{k}(\lambda)$ the $k$-dimensional Jordan block of eigenvalue $\lambda$. Note that the $n$-th power of $J_{k}(\lambda)$ is given by

$$
J_{k}(\lambda)^{n}=\left(\begin{array}{cccc}
{ }_{n} C_{0} \lambda^{n} & { }_{n} C_{1} \lambda^{n-1} & \ldots & { }_{n} C_{k-1} \lambda^{n-k+1} \\
& \ddots & \ddots & \\
& & { }_{n} C_{0} \lambda^{n} & { }_{n} C_{1} \lambda^{n-1} \\
& & & { }_{n} C_{0} \lambda^{n}
\end{array}\right) .
$$

Without loss of generality, the Jordan canonical form $J$ of $X$ is represented as

$$
J=T^{-1} X T=\left(\begin{array}{llll}
J_{k_{1}}\left(\lambda_{1}\right) & & & \\
& J_{k_{2}}\left(\lambda_{2}\right) & & \\
& & \ddots & \\
& & & J_{k_{l}}\left(\lambda_{l}\right)
\end{array}\right)
$$


where $T$ is a nonsingular matrix, $\lambda_{1}, \lambda_{2}, \ldots, \lambda_{l}$ are the eigenvalues of $X$ and $k_{1}, k_{2}, \ldots, k_{l}$ are positive integers satisfying $k_{1}+\cdots+k_{l}=m$. We have

$$
\begin{aligned}
\left(\sum_{n=0}^{\infty}\left|\boldsymbol{a}_{n} X^{n}\right|\right)^{\top} & \leq \sum_{n=0}^{\infty}\left|T^{-1}\right|^{\top}|J|^{n}|T|^{\top}\left|\boldsymbol{a}_{n}\right|^{\top} \\
& =\sum_{n=0}^{\infty}\left(\mathbf{1}^{\top} \otimes\left|T^{-1}\right|^{\top}\right)\left(\operatorname{diag}\left(\left|\boldsymbol{a}_{n}\right|\right) \otimes|J|^{n}\right) \operatorname{vec}\left(|T|^{\top}\right) \\
& =\left(\mathbf{1}^{\top} \otimes\left(\left|T^{-1}\right|\right)^{\top}\right) \operatorname{diag}\left(\sum_{n=0}^{\infty}\left[\left|\boldsymbol{a}_{n}\right|\right]_{j}|J|^{n}, j=1,2, \ldots, s_{0}\right) \operatorname{vec}\left(|T|^{\top}\right),
\end{aligned}
$$

where we use the identity $\operatorname{vec}(A B C)=\left(C^{\top} \otimes A\right) \operatorname{vec}(B)$ for matrices $A, B$ and $C$. Note that

$$
\sum_{n=0}^{\infty}\left[\left|\boldsymbol{a}_{n}\right|\right]_{j}|J|^{n}=\operatorname{diag}\left(\sum_{n=0}^{\infty}\left[\left|\boldsymbol{a}_{n}\right|\right]_{j} J_{k_{s}}\left(\left|\lambda_{s}\right|\right)^{n}, s=1,2, \ldots, l\right) .
$$

and we have, for $t, u \in\left\{1,2, \ldots, k_{s}\right\}$ such that $t \leq u$,

$$
\begin{aligned}
& \sum_{n=0}^{\infty}\left[\left[\left|\boldsymbol{a}_{n}\right|\right]_{j} J_{k_{s}}\left(\left|\lambda_{s}\right|\right)^{n}\right]_{t, u} \\
= & \sum_{n=0}^{k_{s}-2}\left[\left[\left|\boldsymbol{a}_{n}\right|\right]_{j} J_{k_{s}}\left(\left|\lambda_{s}\right|\right)^{n}\right]_{t, u}+\sum_{n=k_{s}-1}^{\infty} \frac{n !}{(u-t) !(n-u+t) !}\left[\left|\boldsymbol{a}_{n}\right|\right]_{j}\left|\lambda_{s}\right|^{n-u+t} \\
\leq & \sum_{n=0}^{k_{s}-2}\left[\left[\left|\boldsymbol{a}_{n}\right|\right]_{j} J_{k_{s}}\left(\left|\lambda_{s}\right|\right)^{n}\right]_{t, u}+\sum_{n=u-t}^{\infty} \frac{n !}{(n-u+t) !}\left[\left|\boldsymbol{a}_{n}\right|\right]_{j}\left|\lambda_{s}\right|^{n-u+t} \\
= & \sum_{n=0}^{k_{s}-2}\left[\left[\left|\boldsymbol{a}_{n}\right|\right]_{j} J_{k_{s}}\left(\left|\lambda_{s}\right|\right)^{n}\right]_{t, u}+\left.\frac{d^{u-t}}{d w^{u-t}}\left[\boldsymbol{\phi}_{a b s}(w)\right]_{j}\right|_{w=\left|\lambda_{s}\right|}
\end{aligned}
$$

where $\boldsymbol{\phi}_{a b s}(w)=\sum_{n=0}^{\infty}\left|\boldsymbol{a}_{n}\right| w^{n}$. For $w \in \mathbb{C}$ such that $|w|<r$, since $\boldsymbol{\phi}(w)$ is absolutely convergent, $\phi_{a b s}(w)$ is also absolutely convergent and analytic. Hence, $\phi_{a b s}(w)$ is differentiable any times and we know that the second term on the last line of formula (B.5.5) is finite since $\left|\lambda_{s}\right| \leq \operatorname{spr}(X)<r$; this completes the proof.

\section{Proof of Lemma 4.1}

Proof. (i) First, we note that if $z$ is a positive real number in $\left[z_{1}^{\min }, z_{1}^{\max }\right]$, then $N_{1}(z), R_{1}(z)$ and $G_{1}(z)$ are finite. Let $z$ be a complex number satisfying $|z| \in\left[z_{1}^{\min }, z_{1}^{\max }\right]$. Since $A_{i, j}, i, j \in \mathbb{H}$, are nonnegative, we have for $j \in \mathbb{H}$,

$$
\left|A_{*, j}(z)\right|=\left|\sum_{i \in \mathbb{H}} A_{i, j} z^{i}\right| \leq \sum_{i \in \mathbb{H}} A_{i, j}|z|^{i}=A_{*, j}(|z|),
$$

and for $n \geq 0$,

$$
\left|Q_{11}^{(n)}(z)\right|=\left|\sum_{\boldsymbol{i}_{(n)} \in \mathscr{I}_{n}} A_{*, i_{1}}(z) A_{*, i_{2}}(z) \cdots A_{*, i_{n}}(z)\right| \leq Q_{11}^{(n)}(|z|) .
$$


From this and the fact that $N_{1}(|z|)=\sum_{n=0}^{\infty} Q_{11}^{(n)}(|z|)$ is finite (convergent), we see that $N_{1}(z)=$ $\sum_{n=0}^{\infty} Q_{11}^{(n)}(z)$ converges absolutely and obtain $\left|N_{1}(z)\right| \leq N_{1}(|z|)$. Analogously, we see that both $G_{1}(z)$ and $R_{1}(z)$ also converge absolutely and satisfy expression (4.1).

(ii) Since, for $n \geq 1, \mathscr{I}_{U, 1, n}$ and $\mathscr{I}_{U, 1, n}$ satisfy

$$
\begin{aligned}
\mathscr{I}_{D, 1, n} & =\left\{\boldsymbol{i}_{(n)} \in \mathbb{H}^{n}: \sum_{l=1}^{k} i_{l} \geq 0 \text { for } k \in\{1,2, \ldots, n-2\}, \sum_{l=1}^{n-1} i_{l}=0 \text { and } i_{n}=-1\right\} \\
& =\left\{\left(\boldsymbol{i}_{(n-1)},-1\right): \boldsymbol{i}_{(n-1)} \in \mathscr{I}_{n-1}\right\}, \\
\mathscr{I}_{U, 1, n} & =\left\{\boldsymbol{i}_{(n)} \in \mathbb{H}^{n}: i_{1}=1, \sum_{l=2}^{k} i_{l} \geq 0 \text { for } k \in\{2, \ldots, n-1\} \text { and } \sum_{l=2}^{n} i_{l}=0\right\} \\
& =\left\{\left(1, \boldsymbol{i}_{(n-1)}\right): \boldsymbol{i}_{(n-1)} \in \mathscr{I}_{n-1}\right\},
\end{aligned}
$$

where $\boldsymbol{i}_{(n)}=\left(i_{1}, i_{2}, \ldots, i_{n}\right)$, we have

$$
\begin{aligned}
& G_{1}(z)=\sum_{n=1}^{\infty} Q_{11}^{(n-1)}(z) A_{*,-1}(z)=N_{1}(z) A_{*,-1}(z) \\
& R_{1}(z)=\sum_{n=1}^{\infty} A_{*, 1}(z) Q_{11}^{(n-1)}(z)=A_{*, 1}(z) N_{1}(z)
\end{aligned}
$$

(iii) We prove only equation (4.3) since equation (4.4) can analogously be proved. For $n \geq 3$, $\mathscr{I}_{D, 1, n}$ satisfies

$$
\begin{aligned}
\mathscr{I}_{D, 1, n}= & \left\{\boldsymbol{i}_{(n)} \in \mathbb{H}^{n}: i_{1}=0, \sum_{l=2}^{k} i_{l} \geq 0 \text { for } k \in\{2, \ldots, n-1\}, \sum_{l=2}^{n} i_{l}=-1\right\} \\
& \bigcup\left\{\boldsymbol{i}_{(n)} \in \mathbb{H}^{n}: i_{1}=1, \sum_{l=2}^{k} i_{l} \geq-1 \text { for } k \in\{2, \ldots, n-1\}, \sum_{l=2}^{n} i_{l}=-2\right\} \\
= & \left\{\left(0, \boldsymbol{i}_{(n-1)}\right): \boldsymbol{i}_{(n-1)} \in \mathscr{I}_{D, 1, n-1}\right\} \cup\left\{\left(1, \boldsymbol{i}_{(n-1)}\right): \boldsymbol{i}_{(n-1)} \in \mathscr{I}_{D, 2, n-1}\right\},
\end{aligned}
$$

and $\mathscr{I}_{D, 2, n}$ satisfies

$$
\begin{aligned}
\mathscr{I}_{D, 2, n}= & \bigcup_{m=1}^{n-1}\left\{\boldsymbol{i}_{(n)} \in \mathbb{H}^{n}: \sum_{l=1}^{k} i_{l} \geq 0 \text { for } k \in\{1,2, \ldots, m-1\}, \sum_{l=1}^{m} i_{l}=-1,\right. \\
& \left.\sum_{l=m+1}^{k} i_{l} \geq 0 \text { for } k \in\{m+1, m+2, \ldots, n-1\} \text { and } \sum_{l=m+1}^{n} i_{l}=-1\right\} \\
= & \bigcup_{m=1}^{n-1}\left\{\left(\boldsymbol{i}_{(m)}, \boldsymbol{i}_{(n-m)}\right): \boldsymbol{i}_{(m)} \in \mathscr{I}_{D, 1, m} \text { and } \boldsymbol{i}_{(n-m)} \in \mathscr{I}_{D, 1, m-n}\right\} .
\end{aligned}
$$


Hence, we have, for $n \geq 3$,

$$
\begin{aligned}
D_{1}^{(n)}(z) & =A_{*, 0}(z) D_{1}^{(n-1)}(z)+A_{*, 1}(z) \sum_{i_{(n-1)} \in \mathscr{I}_{D, 2, n-1}} A_{*, i_{1}}(z) A_{*, i_{2}}(z) \cdots A_{*, i_{n-1}}(z) \\
& =A_{*, 0}(z) D_{1}^{(n-1)}(z)+A_{*, 1}(z) \sum_{m=1}^{n-2} D_{1}^{(m)}(z) D_{1}^{(n-m-1)}(z)
\end{aligned}
$$

and obtain

$$
\begin{aligned}
G_{1}(z) & =D_{1}^{(1)}(z)+\sum_{n=2}^{\infty} A_{*, 0}(z) D_{1}^{(n-1)}(z)+A_{*, 1}(z) \sum_{n=3}^{\infty} \sum_{m=1}^{n-2} D_{1}^{(m)}(z) D_{1}^{(n-m-1)}(z) \\
& =A_{*,-1}(z)+A_{*, 0}(z) G_{1}(z)+A_{*, 1}(z) G_{1}(z)^{2}
\end{aligned}
$$

where we use the fact that $D_{1}^{(1)}(z)=A_{*,-1}(z)$ and $D_{1}^{(2)}(z)=A_{*, 0}(z) D_{1}^{(1)}(z)=A_{*, 0}(z) A_{*,-1}(z)$.

(iv) For $n \geq 1, \mathscr{I}_{n}$ satisfies

$$
\begin{aligned}
& \mathscr{I}_{n}=\left\{\boldsymbol{i}_{(n)} \in \mathbb{H}^{n}: i_{1}=0, \sum_{l=2}^{k} i_{l} \geq 0 \text { for } k \in\{2, \ldots, n-1\}, \sum_{l=2}^{n} i_{l}=0\right\} \\
& \bigcup\left\{\boldsymbol{i}_{(n)} \in \mathbb{H}^{n}: i_{1}=1, \sum_{l=2}^{k} i_{l} \geq-1 \text { for } k \in\{2, \ldots, n-1\}, \sum_{l=2}^{n} i_{l}=-1\right\} \\
&=\left.\left(0, \boldsymbol{i}_{(n-1)}\right): \boldsymbol{i}_{(n-1)} \in \mathscr{I}_{n-1}\right\} \\
& \bigcup_{m=2}^{n}\left\{\boldsymbol{i}_{(n)} \in \mathbb{H}^{n}: i_{1}=1, \sum_{l=2}^{k} i_{l} \geq 0 \text { for } k \in\{2, \ldots, m-1\}, \sum_{l=2}^{m} i_{l}=-1,\right. \\
&\left.\sum_{l=m+1}^{k} i_{l} \geq 0 \text { for } k \in\{m+1, \ldots, n-1\}, \sum_{l=m+1}^{n} i_{l}=0\right\}\left\{\left(0, \boldsymbol{i}_{(n-1)}\right): \boldsymbol{i}_{(n-1)} \in \mathscr{I}_{n-1}\right\} \\
& \cup\left(\cup_{m=2}^{n}\left\{\left(1, \boldsymbol{i}_{(m-1)}, \boldsymbol{i}_{(n-m)}\right): \boldsymbol{i}_{(m-1)} \in \mathscr{I}_{D, 1, m-1}, \boldsymbol{i}_{(n-m)} \in \mathscr{I}_{n-m}\right\}\right) .
\end{aligned}
$$

Hence, we have, for $n \geq 1$,

$$
Q_{1,1}^{(n)}(z)=A_{*, 0}(z) Q_{1,1}^{(n-1)}+\sum_{m=2}^{n} A_{*, 1}(z) D_{1}^{(m-1)}(z) Q_{1,1}^{(n-m)}(z)
$$

and this leads us to

$$
N_{1}(z)=I+A_{*, 0}(z) N_{1}(z)+A_{*, 1}(z) G_{1}(z) N_{1}(z) .
$$

From this equation, we immediately obtain equation (4.5).

(v) Substituting $N_{1}(z) A_{*,-1}(z), A_{*, 1}(z) N_{1}(z)$ and $A_{*, 0}(z)+A_{*, 1}(z) N_{1}(z) A_{*,-1}(z)$ for $G_{1}(z)$, $R_{1}(z)$ and $H_{1}(z)$, respectively, in the right hand side of equation (4.6), we obtain the left hand side of the equation via straightforward calculation. 


\section{Proof of Proposition 4.1}

Proof. Set $z=r_{1} e^{i \theta_{1}}$ and $w=r_{2} e^{i \theta_{2}}$, where $r_{1}, r_{2}>0, \theta_{1}, \theta_{2} \in[0,2 \pi)$ and $i=\sqrt{-1}$. For $n \geq 1$ and $j \in S_{0}, C(z, w)^{n}$ satisfies

$$
\begin{aligned}
\left|\left[C(z, w)^{n}\right]_{j j}\right| & =\mid \sum_{\boldsymbol{k}_{(n)} \in \mathbb{H}^{n}} \sum_{\boldsymbol{l}_{(n)} \in \mathbb{H}^{n}}\left[A_{k_{1}, l_{1}} A_{k_{2}, l_{2}} \cdots A_{k_{n}, l_{n}}\right]_{j j} \\
& \cdot r_{1}^{\sum_{p=1}^{n} k_{p}} r_{2}^{\sum_{p=1}^{n} l_{p}} e^{i\left(\theta_{1} \sum_{p=1}^{n} k_{p}+\theta_{2} \sum_{p=1}^{n} l_{p}\right)} \mid \\
& \leq \sum_{\boldsymbol{k}_{(n)} \in \mathbb{H}^{n}} \sum_{\boldsymbol{l}_{(n)} \in \mathbb{H}^{n}}\left[A_{k_{1}, l_{1}} A_{k_{2}, l_{2}} \cdots A_{k_{n}, l_{n}}\right]_{j j} r_{1}^{\sum_{p=1}^{n} k_{p}} r_{2}^{\sum_{p=1}^{n} l_{p}} \\
& =[C(|z|,|w|)]_{j j},
\end{aligned}
$$

where $\boldsymbol{k}_{(n)}=\left(k_{1}, k_{2}, \ldots, k_{n}\right)$ and $\boldsymbol{l}_{(n)}=\left(l_{1}, l_{2}, \ldots, l_{n}\right)$. In this formula, equality holds only when, for every $\boldsymbol{k}_{(n)}, \boldsymbol{l}_{(n)} \in \mathbb{H}^{n}$ such that $\left[A_{k_{1}, l_{1}} A_{k_{2}, l_{2}} \cdots A_{k_{n}, l_{n}}\right]_{j j} \neq 0, e^{i\left(\theta_{1} \sum_{p=1}^{n} k_{p}+\theta_{2} \sum_{p=1}^{n} l_{p}\right)}$ takes some common value. Consider the Markov chain $\left\{\tilde{\boldsymbol{Y}}_{n}\right\}=\left\{\left(\tilde{X}_{1, n}, \tilde{X}_{2, n}, \tilde{J}_{n}\right)\right\}$ generated by $\left\{A_{k, l}, k, l \in \mathbb{H}\right\}$ (see Definition 2.1) and assume that $\left\{\tilde{\boldsymbol{Y}}_{n}\right\}$ starts from the state $(0,0, j)$. Since $\left\{A_{k, l}, k, l \in \mathbb{H}\right\}$ is irreducible and aperiodic, there exists $n_{0} \geq 1$ such that $\mathbb{P}\left(\tilde{\boldsymbol{Y}}_{n_{0}}=(0,0, j) \mid \tilde{\boldsymbol{Y}}_{0}=(0,0, j)\right)>0$, $\mathbb{P}\left(\tilde{\boldsymbol{Y}}_{n_{0}}=(1,0, j) \mid \tilde{\boldsymbol{Y}}_{0}=(0,0, j)\right)>0$ and $\mathbb{P}\left(\tilde{\boldsymbol{Y}}_{n_{0}}=(0,1, j) \mid \tilde{\boldsymbol{Y}}_{0}=(0,0, j)\right)>0$. This implies that, for some $\boldsymbol{k}_{\left(n_{0}\right)}, \boldsymbol{l}_{\left(n_{0}\right)}, \boldsymbol{k}_{\left(n_{0}\right)}^{\prime}, \boldsymbol{l}_{\left(n_{0}\right)}^{\prime}, \boldsymbol{k}_{\left(n_{0}\right)}^{\prime \prime}$ and $\boldsymbol{l}_{\left(n_{0}\right)}^{\prime \prime}$ in $\mathbb{H}^{n_{0}}$, we have

$$
\begin{aligned}
& {\left[A_{k_{1}, l_{1}} A_{k_{2}, l_{2}} \cdots A_{k_{n_{0}}, l_{n_{0}}}\right]_{j j}>0, \quad \sum_{p=1}^{n_{0}} k_{p}=0, \quad \sum_{p=1}^{n_{0}} l_{p}=0,} \\
& {\left[A_{k_{1}^{\prime}, l_{1}^{\prime}} A_{k_{2}^{\prime}, l_{2}^{\prime}} \cdots A_{k_{n_{0}}^{\prime}, l_{n_{0}}^{\prime}}\right]_{j j}>0, \quad \sum_{p=1}^{n_{0}} k_{p}^{\prime}=1, \quad \sum_{p=1}^{n_{0}} l_{p}^{\prime}=0,} \\
& {\left[A_{k_{1}^{\prime \prime}, l_{1}^{\prime \prime}} A_{k_{2}^{\prime \prime}, l_{2}^{\prime \prime}} \cdots A_{k_{n_{0}}^{\prime \prime}, l_{n_{0}}^{\prime \prime}}\right]_{j j}>0, \quad \sum_{p=1}^{n_{0}} k_{p}^{\prime \prime}=0, \quad \sum_{p=1}^{n_{0}} l_{p}^{\prime \prime}=1 .}
\end{aligned}
$$

Hence, we see that if equality holds in formula (D.1), then $e^{i \theta_{1}}=e^{i \theta_{2}}=1$ and both $\theta_{1}$ and $\theta_{2}$ must be zero. This implies that if $\theta_{1} \neq 0$ or $\theta_{2} \neq 0$, then we have

$$
\left|\left[C(z, w)^{n_{0}}\right]_{j j}\right|=\left|\left[C\left(r_{1} e^{i \theta_{1}}, r_{2} e^{i \theta_{2}}\right)^{n_{0}}\right]_{j j}\right|<\left[C\left(r_{1}, r_{2}\right)^{n_{0}}\right]_{j j}=\left[C(|z|,|w|)^{n_{0}}\right]_{j j},
$$

and obtain

$$
\operatorname{spr}(C(z, w))^{n_{0}} \leq \operatorname{spr}\left(\left|C(z, w)^{n_{0}}\right|\right)<\operatorname{spr}\left(C(|z|,|w|)^{n_{0}}\right)=\operatorname{spr}(C(|z|,|w|))^{n_{0}},
$$

where we use Theorem 1.5 of Seneta [20] and the fact that $C(|z|,|w|))$ is irreducible. Obviously, this implies that $\operatorname{spr}(C(z, w))<\operatorname{spr}(C(|z|,|w|))$.

\section{E Proofs of Propositions 5.1 and 5.2}

Proof of Proposition 5.1. Let $X=\left(x_{k, l}\right)$ be an $s_{0} \times s_{0}$ complex matrix. By Lemma 3.2, if $\operatorname{spr}(X)<$ $r_{2}$, then $\varphi_{2}(X)$ converges absolutely. This means that each element of $\varphi_{2}(X)$ is an absolutely 
convergent series with $s_{0}^{2}$ valuables $x_{11}, x_{12}, \ldots, x_{s_{0}, s_{0}}$ and it is an analytic function with $s_{0}^{2}$ valuables in the region $\left\{X=\left(x_{k, l}\right) \in \mathbb{C}^{s_{0}^{2}}: \operatorname{spr}(X)<r_{2}\right\}$. Therefore, if $G_{1}(z)$ is entry-wise analytic on $\Omega$ and $\operatorname{spr}\left(G_{1}(z)\right)<r_{2}$, we see that $\varphi_{2}\left(G_{1}(z)\right)=\left(\varphi_{2} \circ G_{1}\right)(z)$ is also entry-wise analytic on $\Omega$. For $z \in \mathbb{C}$ such that $|z|<r_{2}$, we have

$$
\sum_{j=1}^{\infty}\left|\boldsymbol{\nu}_{0, j} \hat{C}_{2}\left(z, G_{1}(z)\right) z^{j-1}\right| \leq|z|^{-1} \sum_{j=1}^{\infty} \boldsymbol{\nu}_{0, j}|z|^{j} \hat{C}_{2}\left(|z|, G_{1}(|z|)\right)<\infty .
$$

Hence, by Lemma 3.2, if $\operatorname{spr}(X)<r_{2}, \sum_{j=1}^{\infty} \boldsymbol{\nu}_{0, j} \hat{C}_{2}\left(z, G_{1}(z)\right) X^{j-1}$ converges absolutely, and we see that if $G_{1}(z)$ is entry-wise analytic on $\Omega$ and $\operatorname{spr}\left(G_{1}(z)\right)<r_{2}$, then $\varphi_{2}^{\hat{C}_{2}}\left(z, G_{1}(z)\right)$ is entry-wise analytic on $\Omega$.

Proof of Proposition 5.2. Set $z=r e^{i \theta}$, where $r>0, \theta \in[0,2 \pi)$ and $i=\sqrt{-1}$. By the definition of $G_{1}(z)$, we have

$$
\begin{aligned}
C_{1}\left(z, G_{1}(z)\right)=\sum_{n=1}^{\infty} \sum_{\boldsymbol{k}_{(n+1)} \in \mathbb{H}^{n+1}} \sum_{\boldsymbol{l}_{(n)} \in \mathscr{I}_{D, 1, n}} A_{k_{1}, 1}^{(1)} A_{k_{2}, l_{1}} A_{k_{3}, l_{2}} \cdots A_{k_{n+1}, l_{n}} \\
\cdot r^{\sum_{p=1}^{n+1} k_{p}} e^{i\left(\theta \sum_{p=1}^{n+1} k_{p}\right)}+\sum_{k \in \mathbb{H}} A_{k, 0}^{(1)} r^{k} e^{i \theta k}
\end{aligned}
$$

where $\boldsymbol{k}_{(n+1)}=\left(k_{1}, k_{2}, \ldots, k_{n+1}\right)$ and $\boldsymbol{l}_{(n)}=\left(l_{1}, l_{2}, \ldots, l_{n}\right)$. Consider the Markov chain $\left\{\tilde{\boldsymbol{Y}}_{n}^{(1)}\right\}=$ $\left\{\left(\tilde{X}_{1, n}^{(1)}, \tilde{X}_{2, n}^{(1)}, \tilde{J}_{n}^{(1)}\right)\right\}$ generated by $\left\{\left\{A_{k, l}, k, l \in \mathbb{H}\right\},\left\{A_{k, l}^{(1)}, k \in \mathbb{H}, l \in \mathbb{H}_{+}\right\}\right\}$(see Definition 2.2), and assume that $\left\{\tilde{\boldsymbol{Y}}_{n}^{(1)}\right\}$ starts from a state in $\{0\} \times\{0\} \times S_{0}$. For $n_{0} \in \mathbb{N}$, let $\tau_{n_{0}}$ be the time when the Markov chain enters a state in $\mathbb{Z} \times\{0\} \times S_{0}$ for the $n_{0}$-th time. Then, the term $\sum_{p=1}^{n+1} k_{p}$ (resp. $k$ ) in expression (E.1) indicates that $\tilde{X}_{1, \tau_{1}}^{(1)}=\sum_{p=1}^{n+1} k_{p}$ (resp. $\tilde{X}_{1, \tau_{1}}^{(1)}=k$ ). This point analogously holds for $\tilde{X}_{1, \tau_{n_{0}}}^{(1)}$ when $n_{0}>1$. Hence, $C_{1}\left(z, G_{1}(z)\right)^{n_{0}}$ can be represented as

$$
C_{1}\left(z, G_{1}(z)\right)^{n_{0}}=\sum_{k \in \mathbb{Z}} \tilde{D}_{k} r^{k} e^{i \theta k}
$$

where $k$ indicates that $\tilde{X}_{1, \tau_{n}}^{(1)}=k$ and the $\left(j, j^{\prime}\right)$-entry of nonnegative square matrix $\tilde{D}_{k}$ is given as $\left[\tilde{D}_{k}\right]_{j, j^{\prime}}=\mathbb{P}\left(\tilde{\boldsymbol{Y}}_{\tau_{n_{0}}}^{(1)}=\left(k, 0, j^{\prime}\right) \mid \tilde{\boldsymbol{Y}}_{0}^{(1)}=(0,0, j)\right)$. We have

$$
\left|C_{1}\left(z, G_{1}(z)\right)^{n_{0}}\right|=\left|\sum_{k \in \mathbb{Z}} \tilde{D}_{k} r^{k} e^{i \theta k}\right| \leq C_{1}\left(|z|, G_{1}(|z|)\right)^{n_{0}}
$$

and equality holds only when, for each $j, j^{\prime} \in S_{0}$ and for every $k \in \mathbb{Z}$ such that $\left[\tilde{D}_{k}\right]_{j, j^{\prime}}>0$, $e^{i \theta k}$ takes some common value. Under Assumption [2.2, $\left\{\tilde{\boldsymbol{Y}}_{n}^{(1)}\right\}$ is irreducible and aperiodic, and for any $j, j^{\prime} \in S_{0}$, there exists $n_{0} \geq 1$ such that $\mathbb{P}\left(\tilde{\boldsymbol{Y}}_{\tau_{n_{0}}}^{(1)}=\left(0,0, j^{\prime}\right) \mid \tilde{\boldsymbol{Y}}_{0}^{(1)}=(0,0, j)\right)>0$ and $\mathbb{P}\left(\tilde{\boldsymbol{Y}}_{\tau_{n_{0}}}^{(1)}=\left(1,0, j^{\prime}\right) \mid \tilde{\boldsymbol{Y}}_{0}^{(1)}=(0,0, j)\right)>0$. This implies that $\left[\tilde{D}_{0}\right]_{j j^{\prime}}>0$ and $\left[\tilde{D}_{1}\right]_{j j^{\prime}}>0$. Hence, if $\theta \neq 0(z \neq|z|)$, then we have

$$
\left|\left[C_{1}\left(z, G_{1}(z)\right)^{n_{0}}\right]_{j, j^{\prime}}\right|<\left[C_{1}\left(|z|, G_{1}(|z|)\right)^{n_{0}}\right]_{j, j^{\prime}}
$$


and obtain

$$
\operatorname{spr}\left(C_{1}\left(z, G_{1}(z)\right)^{n_{0}}\right) \leq \operatorname{spr}\left(\left|C_{1}\left(z, G_{1}(z)\right)^{n_{0}}\right|\right)<\operatorname{spr}\left(C_{1}\left(|z|, G_{1}(|z|)\right)^{n_{0}}\right),
$$

where we use Theorem 1.5 of Seneta [20] and the fact that $C_{1}\left(|z|, G_{1}(|z|)\right)$ is irreducible, which is an immediate consequence from Assumption 2.2. Obviously, this implies that $\operatorname{spr}\left(C_{1}\left(z, G_{1}(z)\right)\right)<$ $\operatorname{spr}\left(C_{1}\left(|z|, G_{1}(|z|)\right)\right)$ when $z \neq|z|$.

\section{F Proof of Proposition 5.4}

Proof of Proposition 5.4. Since $\psi_{1}\left(z_{0}\right)=1$, we have

$$
\begin{aligned}
O & =\left(I-C_{1}\left(z_{0}, G_{1}\left(z_{0}\right)\right)\right) \operatorname{adj}\left(I-C_{1}\left(z_{0}, G_{1}\left(z_{0}\right)\right)\right) \\
& =\operatorname{adj}\left(I-C_{1}\left(z_{0}, G_{1}\left(z_{0}\right)\right)\right)\left(I-C_{1}\left(z_{0}, G_{1}\left(z_{0}\right)\right)\right) .
\end{aligned}
$$

Furthermore, $\operatorname{since} \operatorname{rank}\left(I-C_{1}\left(z_{0}, G_{1}\left(z_{0}\right)\right)\right)=s_{0}-1$, we have $\operatorname{adj}\left(I-C_{1}\left(z_{0}, G_{1}\left(z_{0}\right)\right)\right) \neq O$. Hence, for $i, j \in\left\{1,2, \ldots, s_{0}\right\}$, the $i$-th row vector $\boldsymbol{u}_{i}$ and $j$-th column vector $\boldsymbol{v}_{j}$ composing adj $(I-$ $\left.C_{1}\left(z_{0}, G_{1}\left(z_{0}\right)\right)\right)$ are nonzero and satisfy

$$
\boldsymbol{u}_{i} C_{1}\left(z_{0}, G_{1}\left(z_{0}\right)\right)=\boldsymbol{u}_{i}, \quad C_{1}\left(z_{0}, G_{1}\left(z_{0}\right)\right) \boldsymbol{v}_{j}=\boldsymbol{v}_{j} .
$$

Since $C_{1}\left(z_{0}, G_{1}\left(z_{0}\right)\right)$ is irreducible and the algebraic multiplicity of the eigenvalue $\psi_{1}\left(z_{0}\right)$ is one, we see that, for some $s_{0} \times 1$ vector $\boldsymbol{a}=\left(a_{i}\right)$ and $1 \times s_{0}$ vector $\boldsymbol{b}=\left(b_{j}\right)$ and for every $i, j \in$ $\left\{1,2, \ldots, s_{0}\right\}, \boldsymbol{u}_{i}=a_{i} \boldsymbol{u}^{C_{1}}\left(z_{0}\right)$ and $\boldsymbol{v}_{j}=\boldsymbol{v}^{C_{1}}\left(z_{0}\right) b_{j}$. This implies that $\operatorname{adj}\left(I-C_{1}\left(z_{0}, G_{1}\left(z_{0}\right)\right)\right)=$ $\boldsymbol{a u}^{C_{1}}\left(z_{0}\right)=\boldsymbol{v}^{C_{1}}\left(z_{0}\right) \boldsymbol{b}$. From this, we obtain $\boldsymbol{a}=\boldsymbol{a u}^{C_{1}}\left(z_{0}\right) \boldsymbol{v}^{C_{1}}\left(z_{0}\right)=\boldsymbol{v}^{C_{1}}\left(z_{0}\right) \boldsymbol{b} \boldsymbol{u}^{C_{1}}\left(z_{0}\right)$, and $\operatorname{adj}(I-$ $\left.C_{1}\left(z_{0}, G_{1}\left(z_{0}\right)\right)\right)$ is represented as

$$
\operatorname{adj}\left(I-C_{1}\left(z_{0}, G_{1}\left(z_{0}\right)\right)\right)=c \boldsymbol{v}^{C_{1}}\left(z_{0}\right) \boldsymbol{u}^{C_{1}}\left(z_{0}\right),
$$

where $c=\boldsymbol{b} \boldsymbol{u}^{C_{1}}\left(z_{0}\right)$. Note that both $\boldsymbol{u}^{C_{1}}\left(z_{0}\right)$ and $\boldsymbol{v}^{C_{1}}\left(z_{0}\right)$ are positive since $C_{1}\left(z_{0}, G_{1}\left(z_{0}\right)\right)$ is irreducible. Next, we determine the coefficient $c$. For $z<z_{0}$, we have

$$
\left(I-C_{1}\left(z, G_{1}(z)\right)\right)^{-1} \boldsymbol{v}^{C_{1}}(z)=\sum_{k=0}^{\infty} C_{1}\left(z, G_{1}(z)\right)^{k} \boldsymbol{v}^{C_{1}}(z)=\left(1-\psi_{1}(z)\right)^{-1} \boldsymbol{v}^{C_{1}}(z) .
$$

From this and Proposition 5.3, we obtain

$$
\frac{\operatorname{adj}\left(I-C_{1}\left(z_{0}, G_{1}\left(z_{0}\right)\right)\right) \boldsymbol{v}^{C_{1}}(z)}{\psi_{1, z}\left(z_{0}\right) f_{1, \lambda}\left(1, z_{0}\right)}=\lim _{z \uparrow z_{0}}\left(z_{0}-z\right)\left(I-C_{1}\left(z, G_{1}(z)\right)\right)^{-1} \boldsymbol{v}^{C_{1}}(z)=\frac{\boldsymbol{v}^{C_{1}}(z)}{\psi_{1, z}\left(z_{0}\right)},
$$

where we use the fact that $\psi_{1}(z)$ is differentiable on $\left(\underline{z}_{1}^{*}, \bar{z}_{1}^{*}\right)$. Hence, from equation ( $(\overline{\mathrm{F} .3})$, we obtain $c=f_{1, \lambda}\left(1, z_{0}\right)$ and this completes the proof.

\section{G Proof of Proposition 5.6}

Proof of Proposition 5.6. $\tilde{G}_{1,1}$ is given by $\tilde{G}_{1,1}=\left.(d / d \zeta) \tilde{G}_{1}(\zeta)\right|_{\zeta=0}$. Differentiating the both sides of $\tilde{G}_{1}(\zeta)=\tilde{V}_{1}(\zeta) \tilde{J}_{1}(\zeta) \tilde{V}_{1}(\zeta)^{-1}$ and setting $\zeta=0$, we obtain

$$
\tilde{G}_{1,1}=\tilde{\alpha}_{s_{0}, 1}^{G_{1}} \boldsymbol{v}^{\dagger} \boldsymbol{u}_{s_{0}}^{G_{1}}\left(\bar{z}_{1}^{*}\right)
$$


where $\boldsymbol{v}^{\dagger}=\boldsymbol{v}_{s_{0}}^{G_{1}}\left(\bar{z}_{1}^{*}\right)+\left(\tilde{\alpha}_{s_{0}, 1}^{G_{1}}\right)^{-1}\left(\underline{\zeta}_{2}\left(\bar{z}_{1}^{*}\right) I-G_{1}\left(\bar{z}_{1}^{*}\right)\right) \tilde{\boldsymbol{v}}_{s_{0}, 1}$ and $\tilde{\boldsymbol{v}}_{s_{0}, 1}=\left.(d / d \zeta) \tilde{\boldsymbol{v}}_{s_{0}}(\zeta)\right|_{\zeta=0}$. In derivation of equation(G.1), we use the following identity:

$$
\frac{d}{d \zeta} \tilde{V}_{1}(\zeta)^{-1}=-\tilde{V}_{1}(\zeta)^{-1}\left(\frac{d}{d \zeta} \tilde{V}_{1}(\zeta)\right) \tilde{V}_{1}(\zeta)^{-1}
$$

Note that $\boldsymbol{u}_{s_{0}}^{G_{1}}\left(\bar{z}_{1}^{*}\right) \boldsymbol{v}^{\dagger}=1$ and $\tilde{\alpha}_{s_{0}, 1}^{G_{1}}$ is an eigenvalue of $\tilde{G}_{1,1}$. By equation (4.3), $\tilde{G}_{1}(\zeta)$ satisfies

$$
\tilde{G}_{1}(\zeta)=A_{*,-1}\left(\bar{z}_{1}^{*}-\zeta^{2}\right)+A_{*, 0}\left(\bar{z}_{1}^{*}-\zeta^{2}\right) \tilde{G}_{1}(\zeta)+A_{*, 1}\left(\bar{z}_{1}^{*}-\zeta^{2}\right) \tilde{G}_{1}(\zeta)^{2}
$$

Differentiating both the sides of equation (G.2) and setting $\zeta=0$, we obtain

$$
\tilde{G}_{1,1}=A^{\dagger} \tilde{G}_{1,1}
$$

where $A^{\dagger}=A_{*, 0}\left(\bar{z}_{1}^{*}\right)+\underline{\zeta}_{2}\left(\bar{z}_{1}^{*}\right) A_{*, 1}\left(\bar{z}_{1}^{*}\right)+A_{*, 1}\left(\bar{z}_{1}^{*}\right) G_{1}\left(\bar{z}_{1}^{*}\right)$. In derivation of equation (G.3), we use the fact that $\tilde{G}_{1,1} G_{1}\left(\bar{z}_{1}^{*}\right)=\underline{\zeta}_{2}\left(\bar{z}_{1}^{*}\right) \tilde{G}_{1,1}$. Multiplying the both sides of equation (G.3) by $\boldsymbol{v}_{s_{0}}^{G_{1}}\left(\bar{z}_{1}^{*}\right)$ from the right, we obtain $A^{\dagger} \boldsymbol{v}^{\dagger}=\boldsymbol{v}^{\dagger}$. Hence, $\boldsymbol{v}^{\dagger}$ is the right eigenvector of $A^{\dagger}$ with respect to the eigenvalue 1. From equation (4.6) in Lemma 4.1, we obtain

$$
I-A^{\dagger}=\left(\bar{\zeta}_{2}\left(\bar{z}_{1}^{*}\right)^{-1} I-R_{1}\left(\bar{z}_{1}^{*}\right)\right)\left(I-H_{1}\left(\bar{z}_{1}^{*}\right)\right)
$$

and from equation (4.5), we know that $N_{1}\left(\bar{z}_{1}^{*}\right)=\left(I-H_{1}\left(\bar{z}_{1}^{*}\right)\right)^{-1}$. Hence, $N_{1}\left(\bar{z}_{1}^{*}\right) \boldsymbol{v}^{R_{1}}\left(\bar{z}_{1}^{*}\right)$ is the right eigenvector of $A^{\dagger}$ with respect to the eigenvalue 1 , and we see that $\boldsymbol{v}^{\dagger}$ can be given by $\boldsymbol{v}^{\dagger}=N_{1}\left(\bar{z}_{1}^{*}\right) \boldsymbol{v}^{R_{1}}\left(\bar{z}_{1}^{*}\right)$. Since $\boldsymbol{u}_{s_{0}}^{G_{1}}\left(\bar{z}_{1}^{*}\right)$ is the left eigenvector of $\tilde{G}_{1,1}$ with respect to the eigenvalue $\tilde{\alpha}_{s_{0}, 1}^{G_{1}}, \boldsymbol{v}^{R_{1}}\left(\bar{z}_{1}^{*}\right)$ must satisfy $\boldsymbol{u}_{s_{0}}^{G_{1}}\left(\bar{z}_{1}^{*}\right) N_{1}\left(\bar{z}_{1}^{*}\right) \boldsymbol{v}^{R_{1}}\left(\bar{z}_{1}^{*}\right)=1$. Since $\tilde{\alpha}_{s_{0}, 1}^{G_{1}}$ is negative, positivity of $-\tilde{G}_{1,1}$ is obvious. Since $N_{1}\left(\bar{z}_{1}^{*}\right) \geq I$, we have $N_{1}\left(\bar{z}_{1}^{*}\right) \boldsymbol{v}^{R_{1}}\left(\bar{z}_{1}^{*}\right) \geq \boldsymbol{v}^{R_{1}}\left(\bar{z}_{1}^{*}\right) \neq \mathbf{0}$ and $-\tilde{G}_{1,1}$ is nonzero.

\section{H Proof of Proposition 5.7}

Before proving Proposition [5.7, we give the following proposition.

Proposition H.1. In the case of Type II, if $r_{1}<\bar{z}_{1}^{*}$, then $\underline{\zeta}_{2}\left(r_{1}\right)=r_{2}<\bar{z}_{2}^{*}$ and

$$
\lim _{z \rightarrow r_{1}}\left(r_{1}-z\right) \boldsymbol{\varphi}_{2}\left(\alpha_{s_{0}}(z)\right)=\underline{\zeta}_{2, z}\left(r_{1}\right)^{-1} c_{p o l e}^{\boldsymbol{\varphi}_{2}} \boldsymbol{u}^{C_{2}}\left(r_{2}\right)>\mathbf{0}^{\top}
$$

where $\underline{\zeta}_{2, z}(z)=(d / d z) \underline{\zeta}_{2}(z) ;$ if $r_{1}=\bar{z}_{1}^{*}$, then $\underline{\zeta}_{2}\left(\bar{z}_{1}^{*}\right)=r_{2}<\bar{z}_{2}^{*}$ and

$$
\lim _{\tilde{\Delta} \ni z \rightarrow \bar{z}_{1}^{*}}\left(\bar{z}_{1}^{*}-z\right)^{\frac{1}{2}} \boldsymbol{\varphi}_{2}\left(\alpha_{s_{0}}(z)\right)=\left(-\tilde{\alpha}_{s_{0}, 1}^{G_{1}}\right)^{-1} c_{\text {pole }}^{\boldsymbol{\varphi}_{2}} \boldsymbol{u}^{C_{2}}\left(r_{2}\right)>\mathbf{0}^{\top}
$$

Proof. In the case of Type II, we have $\alpha_{s_{0}}\left(r_{1}\right)=\underline{\zeta}_{2}\left(e^{\bar{\eta}_{1}^{(c)}}\right)=e^{\eta_{2}^{(c)}}=r_{2}<\bar{z}_{2}^{*}$, and the point $w=r_{2}$ is a pole of $\varphi_{2}(w)$ with order one. If $r_{1}<\bar{z}_{1}^{*}, \alpha_{s_{0}}(\bar{z})$ is analytic at $z=r_{1}$ and, by Corollary [5.1, we have

$$
\begin{aligned}
\lim _{z \rightarrow r_{1}}\left(r_{1}-z\right) \boldsymbol{\varphi}_{2}\left(\alpha_{s_{0}}(z)\right) & =\lim _{z \rightarrow r_{1}}\left(r_{2}-\alpha_{s_{0}}(z)\right) \boldsymbol{\varphi}_{2}\left(\alpha_{s_{0}}(z)\right) \frac{r_{1}-z}{r_{2}-\alpha_{s_{0}}(z)} \\
& =c_{\text {pole }}^{\boldsymbol{\varphi}_{2}} \boldsymbol{u}^{C_{2}}\left(r_{2}\right) / \underline{\zeta}_{2, z}\left(r_{1}\right)
\end{aligned}
$$


and if $r_{1}=\bar{z}_{1}^{*}$,

$$
\begin{aligned}
\lim _{\tilde{\Delta} \ni z \rightarrow \bar{z}_{1}^{*}}\left(\bar{z}_{1}^{*}-z\right)^{\frac{1}{2}} \boldsymbol{\varphi}_{2}\left(\alpha_{s_{0}}(z)\right) & =\lim _{\tilde{\Delta} \ni z \rightarrow \bar{z}_{1}^{*}}\left(r_{2}-\alpha_{s_{0}}(z)\right) \boldsymbol{\varphi}_{2}\left(\alpha_{s_{0}}(z)\right)\left(-\frac{\left(\bar{z}_{1}^{*}-z\right)^{\frac{1}{2}}}{\alpha_{s_{0}}(z)-r_{2}}\right) \\
& =c_{\text {pole }}^{\boldsymbol{\varphi}_{2}} \boldsymbol{u}^{C_{2}}\left(r_{2}\right) /\left(-\tilde{\alpha}_{s_{0}, 1}^{G_{1}}\right)
\end{aligned}
$$

where we use Proposition 5.5 ,

Proof of Proposition 5.7. In the case of Type I, we always have $\operatorname{spr}\left(G_{1}\left(r_{1}\right)\right)=\underline{\zeta}_{2}\left(r_{1}\right)<r_{2}$. Assume $r_{1}=\bar{z}_{1}^{*}$. By Proposition 5.1, since $\tilde{G}_{1}(0)=\operatorname{spr}\left(G_{1}\left(\bar{z}_{1}^{*}\right)\right)<r_{2}, \tilde{\varphi}_{2}(\zeta)$ is given in a form of absolutely convergent series as

$$
\tilde{\varphi}_{2}\left(\tilde{G}_{1}(\zeta)\right)=\sum_{k=0}^{\infty} \boldsymbol{\nu}_{0, k} \tilde{G}_{1}(\zeta)^{k}
$$

This $\tilde{\varphi}_{2}\left(\tilde{G}_{1}(\zeta)\right)$ is entry-wise analytic at $\zeta=0$ and we have

$$
\begin{aligned}
& \tilde{\boldsymbol{\varphi}}_{2,1}^{\tilde{G}_{1}}=\left.\frac{d}{d \zeta} \tilde{\boldsymbol{\varphi}}_{2}\left(\tilde{G}_{1}(\zeta)\right)\right|_{\zeta=0}=\sum_{k=1}^{\infty} \boldsymbol{\nu}_{0, k} \sum_{l=1}^{k} G_{1}\left(\bar{z}_{1}^{*}\right)^{l-1} \tilde{G}_{1,1} G_{1}\left(\bar{z}_{1}^{*}\right)^{k-l} \\
& =\sum_{k=1}^{\infty} \boldsymbol{\nu}_{0, k} \sum_{l=1}^{k} \underline{\zeta}_{2}\left(\bar{z}_{1}^{*}\right)^{k-l} G_{1}\left(\bar{z}_{1}^{*}\right)^{l-1} \tilde{G}_{1,1}
\end{aligned}
$$

where we use the fact that

$$
\tilde{G}_{1,1} G_{1}\left(\bar{z}_{1}^{*}\right)=\tilde{\alpha}_{s_{0}, 1}^{G_{1}} N_{1}\left(\bar{z}_{1}^{*}\right) \boldsymbol{v}^{R_{1}}\left(\bar{z}_{1}^{*}\right) \boldsymbol{u}_{s_{0}}^{G_{1}}\left(\bar{z}_{1}^{*}\right) G_{1}\left(\bar{z}_{1}^{*}\right)=\underline{\zeta}_{2}\left(\bar{z}_{1}^{*}\right) \tilde{G}_{1,1} .
$$

Since $\tilde{G}_{1,1}$ is nonzero and nonpositive, $\tilde{\varphi}_{2,1}^{\tilde{G}_{1}}$ is also nonzero and nonpositive.

In the case of Type II, we have $\operatorname{spr}\left(G_{1}\left(r_{1}\right)\right)=\underline{\zeta}_{2}\left(r_{1}\right)=r_{2}<\bar{z}_{2}^{*}$ and $\varphi_{2}(w)$ has a pole at $w=\underline{\zeta}_{2}\left(r_{1}\right)$. Hence, if $r_{1}<\bar{z}_{1}^{*}$, we obtain from equation (5.16) and Proposition H.1 that

$$
\begin{aligned}
& \varphi_{2,-1}^{G_{1}}=\lim _{z \rightarrow r_{1}}\left(r_{1}-z\right) \boldsymbol{\varphi}_{2}\left(G_{1}(z)\right) \\
& =\left(\begin{array}{llll}
\mathbf{0} & \cdots & \mathbf{0} & \underline{\zeta}_{2, z}\left(r_{1}\right)^{-1} c_{\text {pole }}^{\boldsymbol{\varphi}_{2}} \boldsymbol{u}^{C_{2}}\left(r_{2}\right) \boldsymbol{v}_{s_{0}}^{G_{1}}\left(r_{1}\right)
\end{array}\right) V_{1}\left(r_{1}\right)^{-1} \\
& =\underline{\zeta}_{2, z}\left(r_{1}\right)^{-1} c_{\text {pole }}^{\boldsymbol{\varphi}_{2}} \boldsymbol{u}^{C_{2}}\left(r_{2}\right) \boldsymbol{v}_{s_{0}}^{G_{1}}\left(r_{1}\right) \boldsymbol{u}_{s_{0}}^{G_{1}}\left(r_{1}\right),
\end{aligned}
$$

where $\boldsymbol{u}^{C_{2}}\left(r_{2}\right) \boldsymbol{v}_{s_{0}}^{G_{1}}\left(r_{1}\right)>0$; if $r_{1}=\bar{z}_{1}^{*}$, we also obtain from equation (5.18) and Proposition H.1 that

$$
\begin{aligned}
& \tilde{\boldsymbol{\varphi}}_{2,-1}^{\tilde{G}_{1}}=\lim _{\tilde{\Delta} \ni z \rightarrow r_{1}}\left(\bar{z}_{1}^{*}-z\right)^{\frac{1}{2}} \boldsymbol{\varphi}_{2}\left(G_{1}(z)\right) \\
& =\left(\begin{array}{llll}
\mathbf{0} & \cdots & \mathbf{0} & \left.\left(-\tilde{\alpha}_{s_{0}}^{G_{1}}\right)^{-1} c_{\text {pole }}^{\boldsymbol{\varphi}_{2}} \boldsymbol{u}^{C_{2}}\left(r_{2}\right) \boldsymbol{v}_{s_{0}}^{G_{1}}\left(\bar{z}_{1}^{*}\right)\right) V_{1}\left(\bar{z}_{1}^{*}\right)^{-1}
\end{array}\right. \\
& =\left(-\tilde{\alpha}_{s_{0}}^{G_{1}}\right)^{-1} c_{\text {pole }}^{\boldsymbol{\varphi}_{2}} \boldsymbol{u}^{C_{2}}\left(r_{2}\right) \boldsymbol{v}_{s_{0}}^{G_{1}}\left(\bar{z}_{1}^{*}\right) \boldsymbol{u}_{s_{0}}^{G_{1}}\left(\bar{z}_{1}^{*}\right),
\end{aligned}
$$

where $\boldsymbol{u}^{C_{2}}\left(r_{2}\right) \boldsymbol{v}_{s_{0}}^{G_{1}}\left(\bar{z}_{1}^{*}\right)>0$. 


\section{Proof of Proposition 5.10}

Proof of Proposition [5.10. We prove only the first half of the proposition. For $z \in\left[\underline{z}_{1}^{*}, \bar{z}_{1}^{*}\right]$, define a nonnegative block tri-diagonal matrix $A_{*}^{(1)}(z)$ as

$$
A_{*}^{(1)}(z)=\left(\begin{array}{ccccc}
A_{*, 0}^{(1)}(z) & A_{*, 1}^{(1)}(z) & & & \\
A_{*,-1}(z) & A_{*, 0}(z) & A_{*, 1}(z) & & \\
& A_{*,-1}(z) & A_{*, 0}(z) & A_{*, 1}(z) & \\
& & \ddots & \ddots & \ddots
\end{array}\right)
$$

Under Assumption 2.2, $A_{*}^{(1)}(z)$ is irreducible and aperiodic. Furthermore, for any $z_{0} \in\left[\underline{z}_{1}^{*}, \bar{z}_{1}^{*}\right]$ such that $\psi_{1}\left(z_{0}\right)=1$, the invariant measure $\boldsymbol{u}_{*}^{(1)}\left(z_{0}\right)$ satisfying $\boldsymbol{u}_{*}^{(1)}\left(z_{0}\right) A_{*}^{(1)}\left(z_{0}\right)=\boldsymbol{u}_{*}^{(1)}\left(z_{0}\right)$ is given as follows (see Theorem 3.1 of Ozawa [18]):

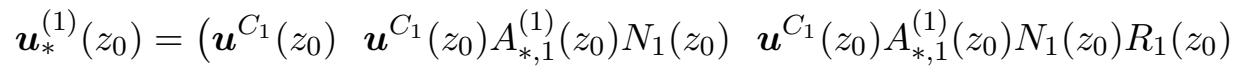

$$
\begin{aligned}
& \left.\boldsymbol{u}^{C_{1}}\left(z_{0}\right) A_{*, 1}^{(1)}\left(z_{0}\right) N_{1}\left(z_{0}\right) R_{1}\left(z_{0}\right)^{2} \quad \ldots\right) \text {. }
\end{aligned}
$$

By Theorem 6.3 of Seneta [20], this $\boldsymbol{u}_{*}^{(1)}\left(z_{0}\right)$ is positive and hence the results of the proposition holds.

\section{J Derivations of the coefficient vectors}

In the case of Type I

(1) $\tilde{\varphi}_{1,-1}^{I}$ of formula (5.40). From Propositions 5.9 and 5.11, we obtain

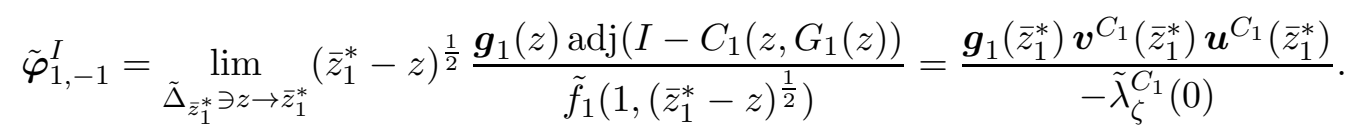

(2) $\tilde{\varphi}_{1,1}^{I}$ of formula (5.42). From Propositions 5.7 and 5.8, we obtain

$$
\begin{aligned}
\tilde{\boldsymbol{\varphi}}_{1,1}^{I}= & \frac{d}{d \zeta} \tilde{\boldsymbol{g}}_{1}(\zeta)\left(I-\left.C_{1}\left(\bar{z}_{1}^{*}-\zeta^{2}, \tilde{G}_{1}(\zeta)\right)^{-1}\right|_{\zeta=0}\right. \\
= & \left(\tilde{\boldsymbol{\varphi}}_{2,1}^{\hat{C}_{2}}-\tilde{\boldsymbol{\varphi}}_{2,1}^{\tilde{G}_{1}}+\boldsymbol{\nu}_{0,0} A_{*, 1}^{(0)}\left(\bar{z}_{1}^{*}\right) \tilde{G}_{1,1}\right)\left(I-C_{1}\left(\bar{z}_{1}^{*}, G_{1}\left(\bar{z}_{1}^{*}\right)\right)\right)^{-1} \\
& \quad+\boldsymbol{g}_{1}\left(\bar{z}_{1}^{*}\right)\left(I-C_{1}\left(\bar{z}_{1}^{*}, G_{1}\left(\bar{z}_{1}^{*}\right)\right)\right)^{-1} A_{*, 1}^{(1)}\left(\bar{z}_{1}^{*}\right) \tilde{G}_{1,1}\left(I-C_{1}\left(\bar{z}_{1}^{*}, G_{1}\left(\bar{z}_{1}^{*}\right)\right)\right)^{-1}
\end{aligned}
$$

and this leads us to formula (5.42).

In the case of Type II

Before deriving expressions for the coefficient vectors, we give the following proposition.

Proposition J.1. In the case of Type II, we have $\operatorname{spr}\left(G_{1}\left(r_{1}\right)\right)=r_{2}, \operatorname{spr}\left(R_{2}\left(r_{2}\right)\right)=r_{1}^{-1}$ and

$$
\boldsymbol{v}_{s_{0}}^{G_{1}}\left(r_{1}\right)=\left(r_{1} I-G_{2}\left(r_{2}\right)\right)^{-1} N_{2}\left(r_{2}\right) \boldsymbol{v}^{R_{2}}\left(r_{2}\right),
$$

where $\boldsymbol{v}^{R_{2}}\left(r_{2}\right)$ is the right eigenvector of $R_{2}\left(r_{2}\right)$ with respect to the eigenvalue $r_{1}^{-1}$. 
Proof. In the case of Type II, we have $\operatorname{spr}\left(G_{1}\left(r_{1}\right)\right)=\underline{\zeta}_{2}\left(r_{1}\right)=r_{2}, \operatorname{spr}\left(R_{1}\left(r_{1}\right)\right)=\bar{\zeta}_{2}\left(r_{1}\right)^{-1} \leq r_{2}^{-1}$, $\operatorname{spr}\left(G_{2}\left(r_{2}\right)\right)=\underline{\zeta}_{1}\left(r_{2}\right)<r_{1}$ and $\operatorname{spr}\left(R_{2}\left(r_{2}\right)\right)=\bar{\zeta}_{1}\left(r_{2}\right)^{-1}=r_{1}^{-1}$. Furthermore, by Lemma 4.1 and Remark 4.1, we have

$$
\begin{aligned}
I-C\left(r_{1}, r_{2}\right) & =\left(r_{2}^{-1} I-R_{1}\left(r_{1}\right)\right)\left(I-H_{1}\left(r_{1}\right)\right)\left(r_{2} I-G_{1}\left(r_{1}\right)\right) \\
& =\left(r_{1}^{-1} I-R_{2}\left(r_{2}\right)\right)\left(I-H_{2}\left(r_{2}\right)\right)\left(r_{1} I-G_{2}\left(r_{2}\right)\right) .
\end{aligned}
$$

Multiplying both the sides of this equation by $\boldsymbol{v}_{s_{0}}^{G_{1}}\left(r_{1}\right)$ from the right, we obtain

$$
\left(r_{1}^{-1} I-R_{2}\left(r_{2}\right)\right)\left(I-H_{2}\left(r_{2}\right)\right)\left(r_{1} I-G_{2}\left(r_{2}\right)\right) \boldsymbol{v}_{s_{0}}^{G_{1}}\left(r_{1}\right)=\mathbf{0} .
$$

Since both $\left(I-H_{2}\left(r_{2}\right)\right)$ and $\left(r_{1} I-G_{2}\left(r_{2}\right)\right)$ are nonsingular, we obtain

$$
\boldsymbol{v}^{R_{2}}\left(r_{2}\right)=\left(I-H_{2}\left(r_{2}\right)\right)\left(r_{1} I-G_{2}\left(r_{2}\right)\right) \boldsymbol{v}_{s_{0}}^{G_{1}}\left(r_{1}\right) \neq \mathbf{0}
$$

and this leads us to expression ( (J.1) , where we use the fact that $N_{2}\left(r_{2}\right)=\left(I-H_{2}\left(r_{2}\right)\right)^{-1}$.

(1) $\varphi_{1,-1}^{I I}$ of formula (5.44). Note that we have

$$
\begin{aligned}
\boldsymbol{u}^{C_{2}}\left(r_{2}\right)\left(C_{2}\left(r_{1}, r_{2}\right)-I\right) & =\boldsymbol{u}^{C_{2}}\left(r_{2}\right)\left(C_{2}\left(r_{1}, r_{2}\right)-C_{2}\left(G_{2}\left(r_{2}\right), r_{2}\right)\right) \\
& =\boldsymbol{u}^{C_{2}}\left(r_{2}\right) A_{1, *}^{(2)}\left(r_{2}\right)\left(r_{1} I-G_{2}\left(r_{2}\right)\right) .
\end{aligned}
$$

Hence, from Propositions 5.7 and 5.8, we obtain

$$
\begin{aligned}
\boldsymbol{\varphi}_{1,-1}^{I I} & =\lim _{z \rightarrow r_{1}}\left(r_{1}-z\right) \boldsymbol{g}_{1}(z)\left(I-C_{1}\left(z, G_{1}(z)\right)^{-1}\right. \\
& =\left(\boldsymbol{\varphi}_{2,-1}^{\hat{C}_{2}}-\boldsymbol{\varphi}_{2,-1}^{\tilde{G}_{1}}\right)\left(I-C_{1}\left(r_{1}, G_{1}\left(r_{1}\right)\right)\right)^{-1} \\
& =\frac{c_{\text {pole }}^{\boldsymbol{\varphi}_{2}} \boldsymbol{u}^{C_{2}}\left(r_{2}\right) A_{1, *}^{(2)}\left(r_{2}\right)\left(r_{1} I-G_{2}\left(r_{2}\right)\right) \boldsymbol{v}_{s_{0}}^{G_{1}}\left(r_{1}\right) \boldsymbol{u}_{s_{0}}^{G_{1}}\left(r_{1}\right)\left(I-C_{1}\left(r_{1}, G_{1}\left(r_{1}\right)\right)\right)^{-1}}{\underline{\zeta}_{2, z}\left(r_{1}\right)} .
\end{aligned}
$$

This and Proposition J.1 lead us to formula (5.44).

(2) $\varphi_{1,-2}^{I I}$ of formula (5.46). From Propositions 5.3, 5.4, 5.7 and 5.8, we obtain

$$
\begin{aligned}
\boldsymbol{\varphi}_{1,-2}^{I I} & =\lim _{z \rightarrow r_{1}}\left(r_{1}-z\right)^{2} \frac{\boldsymbol{g}_{1}(z) \operatorname{adj}\left(I-C_{1}\left(z, G_{1}(z)\right)\right)}{f_{1}(1, z)} \\
& =\frac{\left(\boldsymbol{\varphi}_{2,-1}^{\hat{C}_{2}}-\boldsymbol{\varphi}_{2,-1}^{\tilde{G}_{1}}\right) \boldsymbol{u}^{C_{1}}\left(r_{1}\right) \boldsymbol{v}^{C_{1}}\left(r_{1}\right)}{\psi_{1, z}\left(r_{1}\right)} \\
& =\frac{c_{p o l e}^{\boldsymbol{\varphi}_{2}} \boldsymbol{u}^{C_{2}}\left(r_{2}\right) A_{1, *}^{(2)}\left(r_{2}\right)\left(r_{1} I-G_{2}\left(r_{2}\right)\right) \boldsymbol{v}_{s_{0}}^{G_{1}}\left(r_{1}\right) \boldsymbol{u}_{s_{0}}^{G_{1}}\left(r_{1}\right) \boldsymbol{v}^{C_{1}}\left(r_{1}\right) \boldsymbol{u}^{C_{1}}\left(r_{1}\right)}{\underline{\zeta}_{2, z}\left(r_{1}\right) \psi_{1, z}\left(r_{1}\right)}
\end{aligned}
$$

This and Proposition J.1 lead us to formula (5.46). 
(3) $\tilde{\varphi}_{1,-2}^{I I}$ of formula (5.48). From Propositions [5.7, 5.8, 5.9] and [5.11, we obtain

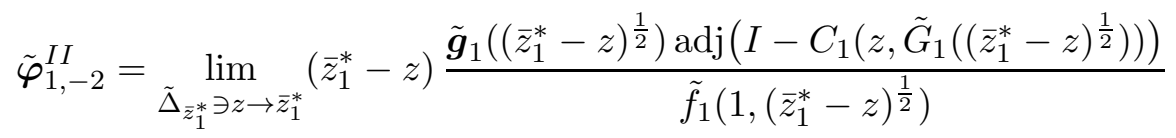

$$
\begin{aligned}
& =\frac{\left(\tilde{\boldsymbol{\varphi}}_{2,-1}^{\hat{C}_{2}}-\tilde{\boldsymbol{\varphi}}_{2,-1}^{\tilde{G}_{1}}\right) \boldsymbol{u}^{C_{1}}\left(\bar{z}_{1}^{*}\right) \boldsymbol{v}^{C_{1}}\left(\bar{z}_{1}^{*}\right)}{-\tilde{\lambda}_{\zeta}^{C_{1}}(0)} \\
& =\frac{c_{p o l e}^{\boldsymbol{\varphi}_{2}} \boldsymbol{u}^{C_{2}}\left(r_{2}\right) A_{1, *}^{(2)}\left(r_{2}\right)\left(\bar{z}_{1}^{*} I-G_{2}\left(r_{2}\right)\right) \boldsymbol{v}_{s_{0}}^{G_{1}}\left(\bar{z}_{1}^{*}\right) \boldsymbol{u}_{s_{0}}^{G_{1}}\left(\bar{z}_{1}^{*}\right) \boldsymbol{v}^{C_{1}}\left(\bar{z}_{1}^{*}\right) \boldsymbol{u}^{C_{1}}\left(\bar{z}_{1}^{*}\right)}{\tilde{\alpha}_{s_{0}, 1}^{G_{1}} \tilde{\lambda}_{\zeta}^{C_{1}}(0)} .
\end{aligned}
$$

This and Proposition J.1 lead us to formula (5.48).

(4) $\tilde{\varphi}_{1,-1}^{I I}$ of formula (5.50). From Propositions 5.7 and 5.8, we obtain

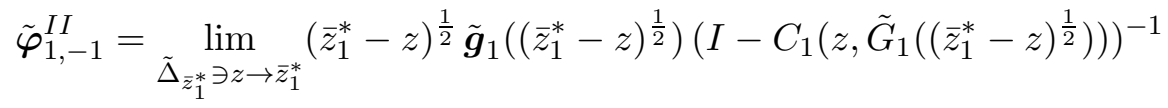

$$
\begin{aligned}
& =\left(\tilde{\varphi}_{2,-1}^{\hat{C}_{2}}-\tilde{\varphi}_{2,-1}^{\tilde{G}_{1}}\right)\left(I-C_{1}\left(\bar{z}_{1}^{*}, G_{1}\left(\bar{z}_{1}^{*}\right)\right)\right)^{-1} \\
& =\frac{c_{\text {pole }}^{\boldsymbol{\varphi}_{2}} \boldsymbol{u}^{C_{2}}\left(r_{2}\right) A_{1, *}^{(2)}\left(r_{2}\right)\left(\bar{z}_{1}^{*} I-G_{2}\left(r_{2}\right)\right) \boldsymbol{v}_{s_{0}}^{G_{1}}\left(\bar{z}_{1}^{*}\right) \boldsymbol{u}_{s_{0}}^{G_{1}}\left(\bar{z}_{1}^{*}\right)\left(I-C_{1}\left(\bar{z}_{1}^{*}, G_{1}\left(\bar{z}_{1}^{*}\right)\right)\right)^{-1}}{-\tilde{\alpha}_{s_{0}, 1}^{G_{1}}} .
\end{aligned}
$$

This and Proposition J.1 lead us to formula (5.50). 